\title{
Central venous catheters in hematological patients : risky lifelines?
}

Citation for published version (APA):

Boersma, R. S. (2015). Central venous catheters in hematological patients : risky lifelines ? [Doctoral Thesis, Maastricht University]. Datawyse / Universitaire Pers Maastricht. https://doi.org/10.26481/dis.20151120rb

Document status and date:

Published: 01/01/2015

DOI:

10.26481/dis.20151120rb

Document Version:

Publisher's PDF, also known as Version of record

\section{Please check the document version of this publication:}

- A submitted manuscript is the version of the article upon submission and before peer-review. There can be important differences between the submitted version and the official published version of record.

People interested in the research are advised to contact the author for the final version of the publication, or visit the DOI to the publisher's website.

- The final author version and the galley proof are versions of the publication after peer review.

- The final published version features the final layout of the paper including the volume, issue and page numbers.

Link to publication

\footnotetext{
General rights rights.

- You may freely distribute the URL identifying the publication in the public portal. please follow below link for the End User Agreement:

www.umlib.nl/taverne-license

Take down policy

If you believe that this document breaches copyright please contact us at:

repository@maastrichtuniversity.nl

providing details and we will investigate your claim.
}

Copyright and moral rights for the publications made accessible in the public portal are retained by the authors and/or other copyright owners and it is a condition of accessing publications that users recognise and abide by the legal requirements associated with these

- Users may download and print one copy of any publication from the public portal for the purpose of private study or research.

- You may not further distribute the material or use it for any profit-making activity or commercial gain

If the publication is distributed under the terms of Article $25 \mathrm{fa}$ of the Dutch Copyright Act, indicated by the "Taverne" license above, 


\section{Central venous catheters in hematological patients}

Risky lifelines?

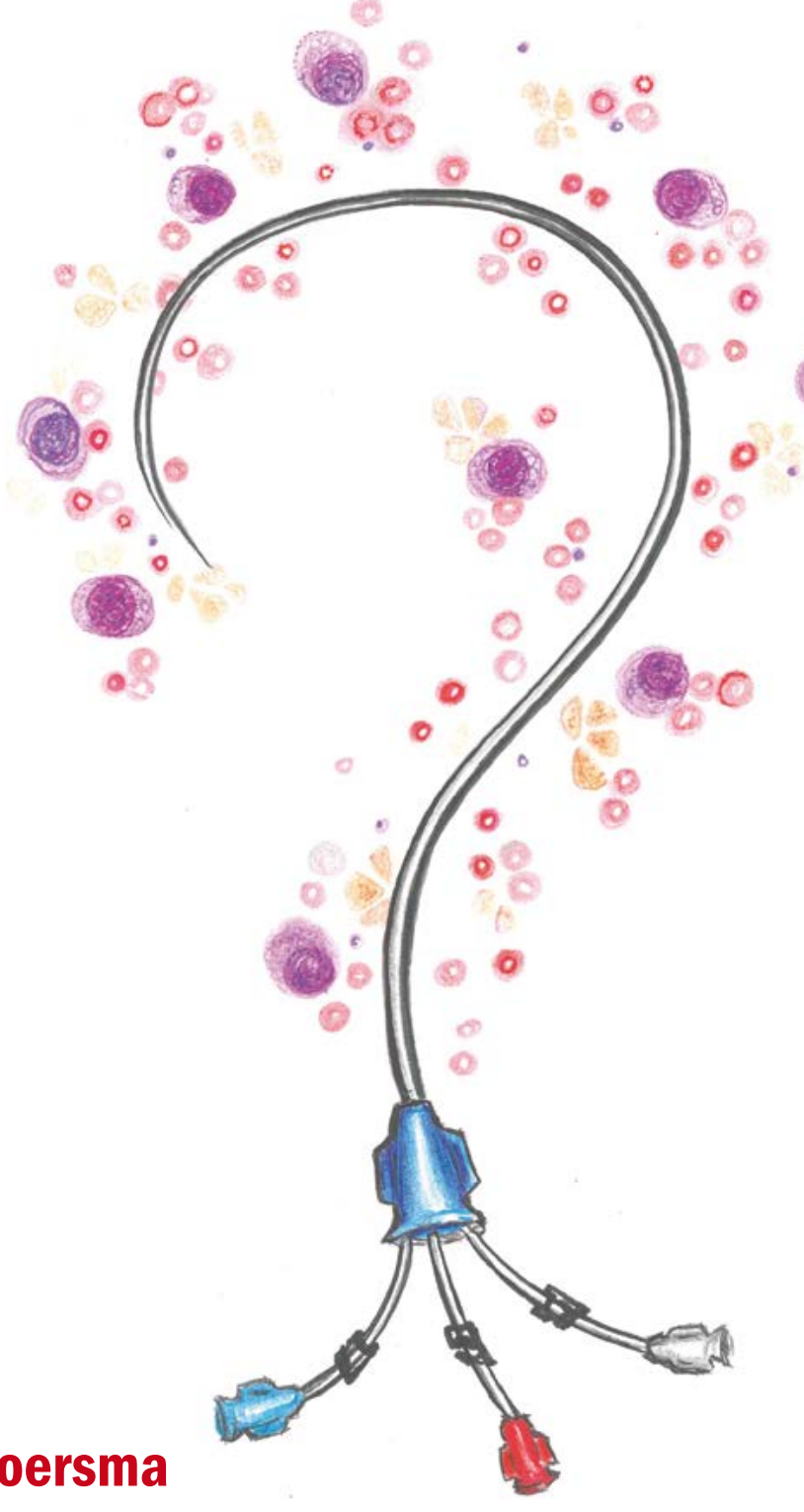


(C) Rinske Sandrien Boersma

Cover: Barbara Broeren-van Loon

ISBN: 9789461594860

Production: Datawyse | Universitaire Pers Maastricht 


\title{
Central venous catheters in hematological patients
}

\author{
Risky lifelines?
}

\author{
PROEFSCHRIFT \\ Ter verkrijging van de graad van doctor \\ aan de Universiteit Maastricht, \\ op gezag van de \\ Rector Magnificus, Prof. Dr. L. L. G. Soete \\ volgens het besluit van het College van Decanen, \\ in het openbaar te verdedigen \\ op vrijdag 20 november 2015 om 16:00 uur \\ door
}

Rinske Sandrien Boersma

Geboren op 4 september 1978 te Velp

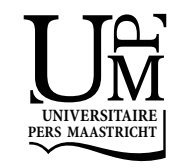


Promotor

Prof. dr. H. C. Schouten

\section{Copromotor}

Dr. K. Hamulyak

\section{Beoordelingscommissie}

Prof. dr. G. M. J. Bos (voorzitter)

Dr. M. R. Nijziel (Maxima Medisch Centrum Eindhoven)

Prof. dr. C. D. A. Stehouwer

Dr. P. J. Voogt (Zuyderland Medisch Centrum) 


\section{Contents}

Chapter 1 General introduction

Chapter 2 Thrombotic and infectious complications of central venous

catheters in patients with hematological malignancies

Chapter 3 Clinical practices concerning central venous catheters in hematological patients

Chapter 4 Concentrated citrate locking in order to reduce the long term complications of central venous catheters: a randomized controlled trial in patients with hematological malignancies

Chapter 5 Congenital thrombophilia and central venous catheter- related thrombosis in patients with cancer

Chapter 6 Biomarkers for prediction of central venous catheter relatedthrombosis in patients with hematological malignancies

Chapter 7 General conclusions and further perspectives

Summary

Nederlandstalige samenvatting

Dankwoord

Curriculum vitae

List of publications

Valorization 

General introduction

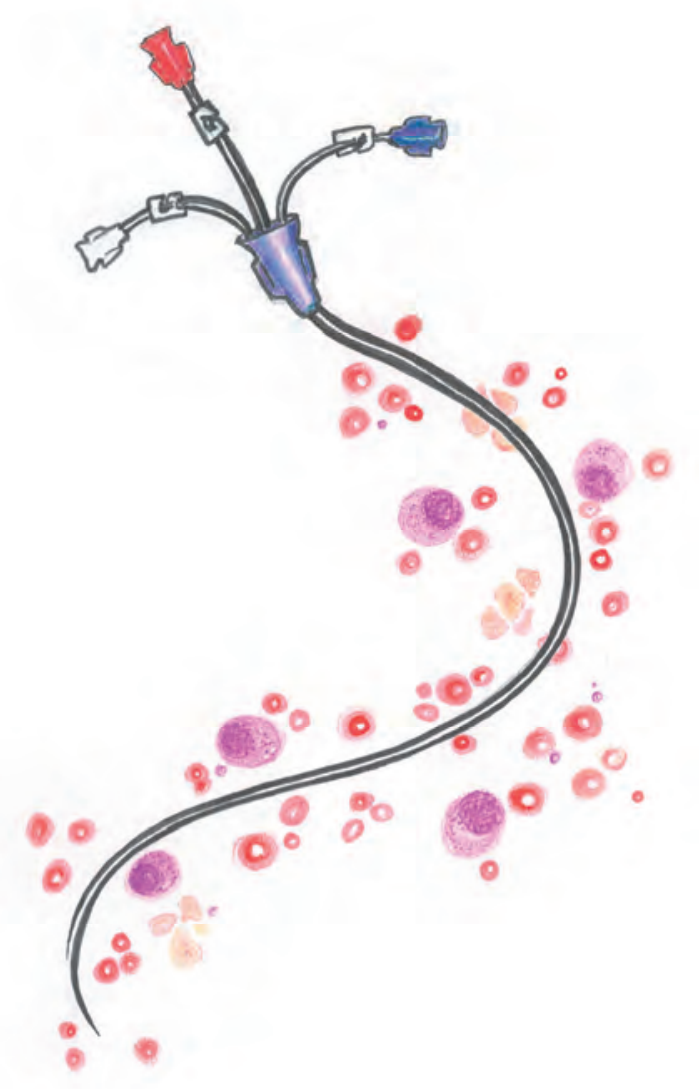





\section{CENTRAL VENOUS CATHETERS IN HEMATOLOGICAL PATIENTS RISKY LIFELINES?}

A central venous catheter (CVC) is a catheter type inserted into large veins, such as the subclavian vein, internal jugular vein, femoral vein or basilic vein. To qualify as a central venous catheter the device must terminate in one of the great vessels in or near the heart. The history of central venous cannulation starts in 1929 when Forssmann described the advance of a plastic tube to the heart by puncturing his own arm vein. At the beginning of the 1950s Aubaniac reported about the puncture of the subclavian vein. Since this time central venous catheterization has developed to a standard procedure in clinical practice [1].

Since its introduction, many types of central venous catheters are now available [2]. They vary in design, material and insertion requirements, but they share similar advantages and complications.

These central venous catheters are grouped into: 1) tunneled catheters with an anchoring cuff; 2) non-tunneled catheters; 3) implanted ports; 4) peripherally inserted central catheters (PICCS) and 5) apheresis or dialysis catheters. They can have single, double or triple lumens with valved or open ends (figure 1). Valves prevent the reflux of blood into the catheter and claime to obviate the need to use heparinized solutions for flushing. The choice for a central venous catheter depends on the clinical need [3].

Physicians have gradually been improving the technique for inserting central venous catheters. Today, the Seldinger technique (guide wire technique) is the method of choice. Most CVCs are inserted with the help of ultrasound [4, 5]. All long-term central venous catheters are designed to have the distal catheter tip indwelling in the central venous system at the junction of the superior vena cava and the right atrium (figure 2). Having the tip at the junction of the superior vena cava and right atrium may be protective because there is a lower likelihood that the tip of the CVC will be in direct contact with the endothelium and because of a greater dilutional effect when chemotherapeutic agents are infused.

CVCs are extensively used in patients with hematological malignancies undergoing intensive chemotherapy to provide cancer treatment and supportive care therapies. These CVCs improve patients' quality of life by reducing the need for venipunctures and allowing patients to receive chemotherapy, stem cell infusions, blood products, medication and parenteral nutrition without peripheral access. Most patients with hematological malignancies undergoing intensive chemotherapy need (non) tunneled central venous catheters with double or triple lumens.

The great benefit derived from CVCs can be offset by complications. Reported complications consist of mechanical complications during or directly after the insertion (arterial puncture, hematoma and pneumothorax; table 1) and long-term complications like CVC-related infections and thrombosis [6]. 
CVC-related infections and thrombosis impair CVC functioning and result in patient morbidity, may interrupt or delay administration of therapy, increase the risk of CVCrelated thrombosis and/or infections and represent a costly burden to the healthcare system [7-9]. Long-term complications pose hematologists with difficult questions on what anticoagulant treatment to choose in often thrombocytopenic patients and whether the CVC must be removed.

Anticipation of the risk of CVC-related thrombosis and the identification of "highrisk" patients who are prone to develop CVC-related thrombosis is essential to initiate early preventive measures.

CVC-related infections and thrombosis should not be considered as a result of modern care but should be one of the priority targets of a multidisciplinary approach emphasizing quality-of-care improvement.

Table 1: Early complications of central venous catheters, according to the route of catheterization [6]

\begin{tabular}{llll}
\hline Complication & \multicolumn{2}{l}{ Frequency } & \\
\cline { 2 - 4 } & Internal Jugular \% & Subclavian \% & Femoral \% \\
\hline Arterial puncture & $6.3-9.4$ & $3.1-4.9$ & $9.0-15.0$ \\
Hematoma & $<0.1-2.2$ & $1.2-2.1$ & $3.8-4.4$ \\
Hemothorax & $\mathrm{NA}$ & $0.4-0.6$ & $\mathrm{NA}$ \\
Pneumothorax & $<0.1-0.2$ & $1.5-3.1$ & $\mathrm{NA}$ \\
Total & $6.3-11.8$ & $6.2-10.7$ & $12.8-19.4$ \\
\hline
\end{tabular}
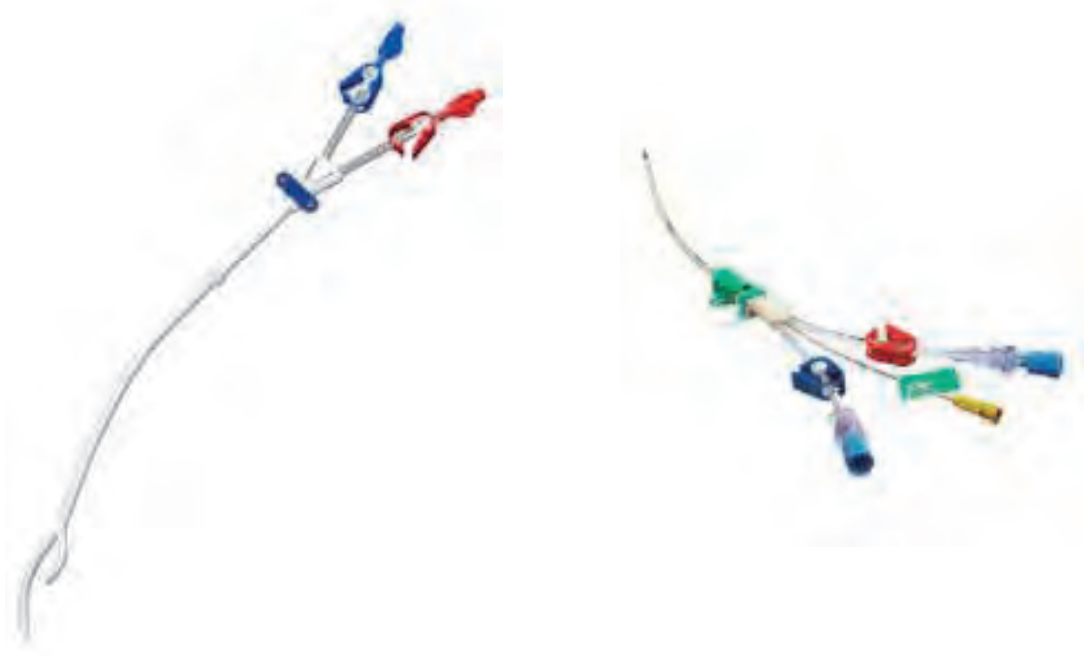

Figure 1. picture of a double lumen and triple lumen central venous catheter 


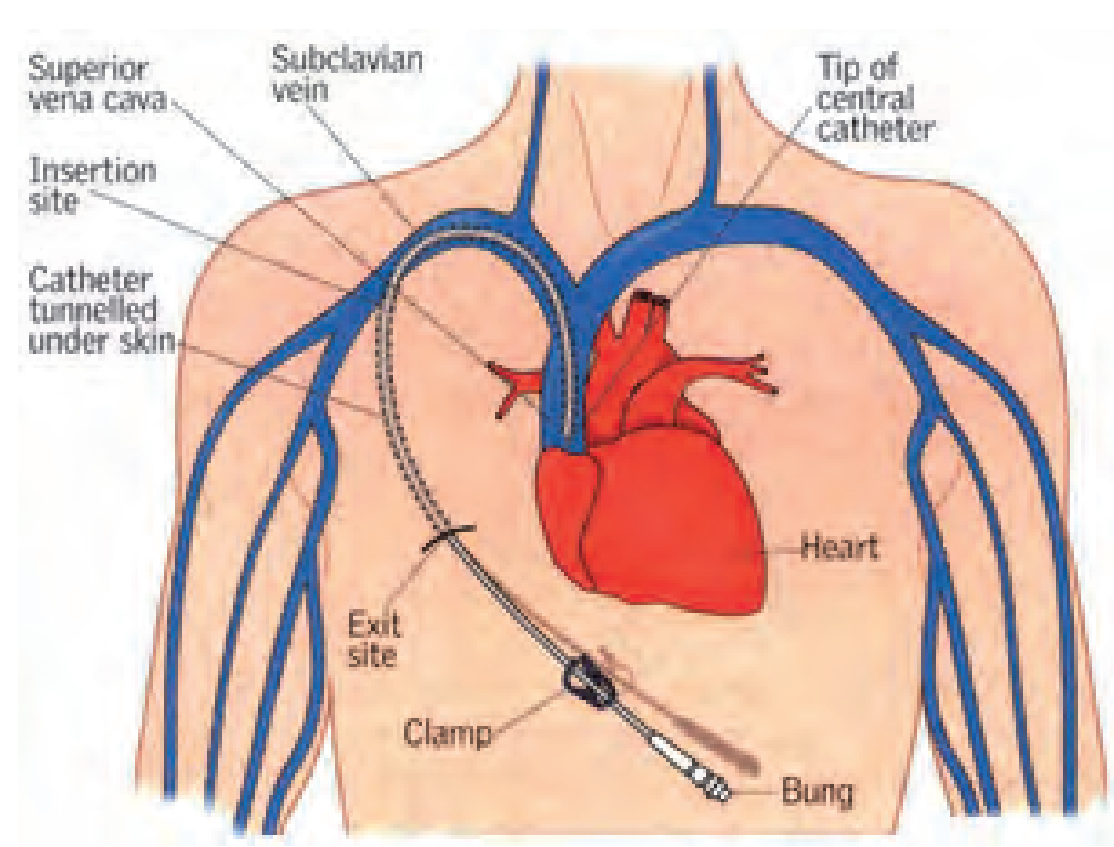

Figure 2. picture of a central venous catheter inserted in the right jugular vein with the tip positioned at the junction of the vena cava superior and right atrium

This thesis addresses several major topics concerning complications of central venous catheters in hematological patients undergoing intensive chemotherapy.

In chapter 2 the incidence, pathogenesis, risk factors, diagnosis, prevention and treatment of CVC-related infections and thrombosis in hematological patients undergoing intensive chemotherapy are reviewed and discussed. Chapter 2 is based on a review published in 2008 and updated in April 2015.

In chapter 3 we describe a survey conducted among 23 Dutch and Belgian hematological centres to assess the local views and clinical practices concerning central venous catheters in hematological patients undergoing intensive chemotherapy. The goal of the survey was to evaluate the extent of variation in practice and to promote further research.

In chapter 4 we describe a prospective, multicenter, randomized controlled study that was performed to determine the efficacy of trisodium citrate (TSC) as a locking solution compared to heparin in preventing CVC-related infections and thrombosis in patients with hematological malignancies undergoing intensive chemotherapy. The study was conducted between July 2006 and August 2010 at the University Hospital Maastricht and the Atrium Medical Center Parkstad Heerlen, The Netherlands.

In chapter 5 we reviewed whether a state of congenital thrombophilia increases the risk of CVC-related thrombosis in patients with cancer. The studies were retrieved by an extensive Medline search. 
In chapter 6 we evaluate associations between laboratory biomarkers and the occurrence of symptomatic CVC-related thrombosis in 168 patients with hematological malignancies undergoing intensive chemotherapy.

In chapter 7, the most important findings are summarized and discussed within the context of recent literature. Limitations of this thesis are mentioned, and recommendations for future research are given. 


\section{REFERENCES}

1. Kalso E. A short history of central venous catheterization. Acta Anaesthesiol Scand Suppl 1985; 81: 7-10.

2. Broviac JW, Cole JJ, Scribner BH. A silicone rubber atrial catheter for prolonged parenteral alimentation. Surg Gynecol Obstet 1973; 136: 602-606.

3. Kamphuisen PW, Lee AY. Catheter-related thrombosis: lifeline or a pain in the neck? Hematology Am Soc Hematol Educ Program 2012: 638-644.

4. Brass $P$, Hellmich $M$, Kolodziej $L$ et al. Ultrasound guidance versus anatomical landmarks for subclavian or femoral vein catheterization. Cochrane Database Syst Rev 2015; 9: CD011447.

5. Cavanna L, Civardi G, Vallisa D et al. Ultrasound-guided central venous catheterization in cancer patients improves the success rate of cannulation and reduces mechanical complications: a prospective observational study of 1,978 consecutive catheterizations. World J Surg Oncol 2014; 8: 91.

6. McGee DC, Gould MK. Preventing complications of central venous catheterization. N Engl J Med 2003; 348: 1123-1133.

7. Pittet D, Tarara D, Wenzel RP. Nosocomial bloodstream infection in critically ill patients. Excess length of stay, extra costs, and attributable mortality. Jama 1994; 271: 1598-1601.

8. Jarvis WR. Selected aspects of the socioeconomic impact of nosocomial infections: morbidity, mortality, cost, and prevention. Infect Control Hosp Epidemiol 1996; 17: 552-557.

9. Raad I, Chaftari AM. Advances in prevention and management of central line-associated bloodstream infections in patients with cancer. Clin Infect Dis 2014; 59 Suppl 5: S340-343. 



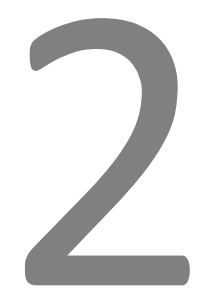

Thrombotic and infectious

complications of central

venous catheters in patients

with hematological malignancies

R.S. Boersma, K.S. Jie, A. Verbon, E.C.M. van Pampus, H.C. Schouten

Based on Ann Oncol. 2008 Mar;19(3): 433-442 and revised in April 2015
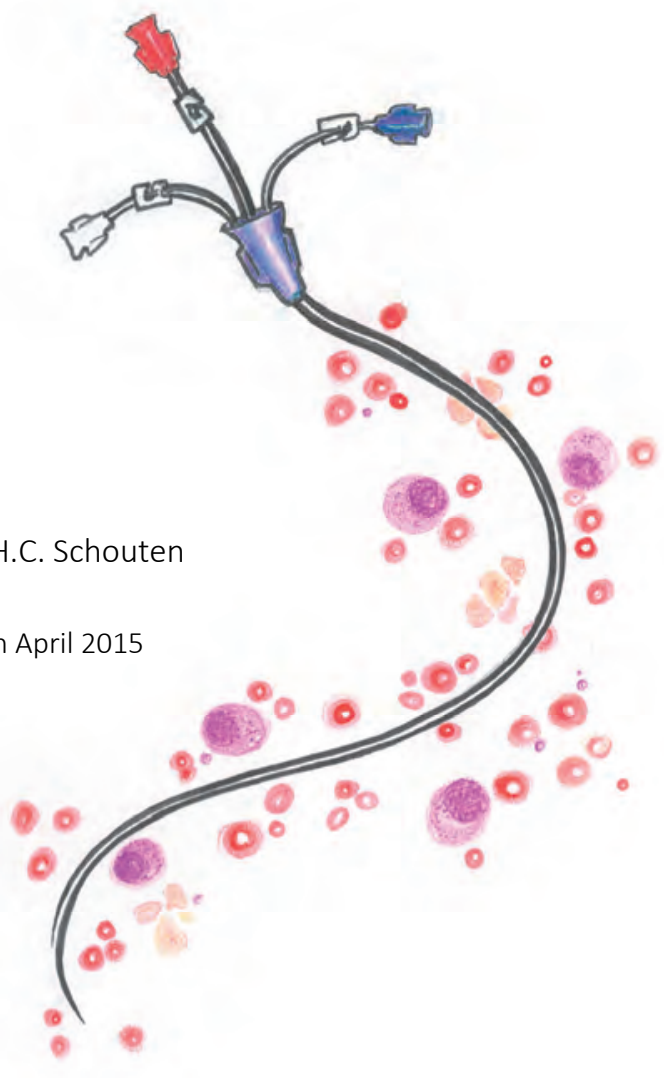


\section{ABSTRACT}

Central venous catheters (CVCs) have considerably improved the management of patients with hematological malignancies, by facilitating chemotherapy, supportive therapy and blood sampling. Complications of insertion of CVCs include mechanical (arterial puncture, pneumothorax), thrombotic and infectious complications. CVCrelated thrombosis and infections are frequently occurring complications and may cause significant morbidity in patients with hematological malignancies. CVC-related thrombosis and infections are related and can therefore not be seen as separate entities. The incidence of symptomatic CVC-related thrombosis has been reported to vary between 1.2 and $18.0 \%$ of patients with a hematological malignancy. The incidence of CVC-related bloodstream infections varies between 0.0 and $40.6 \%$. There is need for a specific approach regarding diagnosis and treatment of CVC-related thrombosis and infections with specific attention for the preservation of the catheter. Since data on CVC-related infections and thrombosis in hematological patients have been obtained mainly from retrospective studies of small sample size, prospective, randomized studies of prophylactic measures concerning CVC-related thrombosis and infections are warranted. 


\section{INTRODUCTION}

Central venous catheters (CVCs) are frequently used in patients with a hematological malignancy in order to receive chemotherapy, stem cell infusions, blood products, medication, parenteral hyperalimentation as well as for blood sampling all along the course of the disease. In addition CVCs are helpful in some therapeutic procedures, such as stem cell collection and apheresis.

Reported complications consist of mechanical complications during or directly after the insertion (arterial puncture, hematoma, and pneumothorax), and long-term complications such as infections and thrombosis [1]. Central venous catheter-related thrombosis and infections result in patient morbidity, significant increases in the length of hospitalization and medical care costs [2-4].

Many studies have addressed the incidence and associated risk factors of CVCrelated infections and thrombosis in patients with solid tumors, but only few data are available on hematological patients. These patients differ from patients with solid tumors with respect to more severe and prolonged thrombocytopenia and leucopenia $[5,6]$. Thrombocytopenia is associated with a trend for reduced risk of thrombotic complications [5], but might increase the risk of bleeding and perhaps complicates the use of antithrombotic prophylaxis and treatment. Patients with severe and sustained neutropenia are at high risk for infectious complications.

Since CVC-related thrombosis and CVC-related infections cannot be seen as separate entities, this review focuses on the epidemiology, pathogenesis, diagnosis, prevention and treatment of both CVC-related thrombosis and CVC-related infections in patients with a hematological malignancy.

\section{CVC-RELATED THROMBOSIS}

CVC-related thrombosis is defined as a mural thrombus extending from the CVC into the lumen of a vessel, and leading to partial or total catheter occlusion with or without clinical symptoms [7].

CVC-related thrombosis may be asymptomatic and only demonstrated by screening diagnostic imaging or present with symptoms. An asymptomatic CVC-related thrombosis is found in $2.0 \%-34.1 \%$ of patients with a hematological malignancy (see table 1). About two third of all thromboses are clinically silent. The presence of asymptomatic thrombosis increases the risk of developing symptomatic thrombosis sevenfold compared with negative Doppler ultrasound findings (RR 6.8; 95\% Cl 2.3-20.2) [8]. Asymptomatic thrombosis may be clinically important.

A strong association between asymptomatic CVC-related thrombosis and catheter complications like occlusion of the CVC and CVC-related infections has been reported [9, 10]. 
Table 1. Incidence of CVC-related thrombosis in hematological patients

\begin{tabular}{|c|c|c|c|c|c|c|}
\hline Author & Year & Study design & No CVC & $\begin{array}{l}\text { CVC- related } \\
\text { thrombosis }\end{array}$ & (a)symptomatic & CVC care \\
\hline \multirow[t]{2}{*}{ Boraks et al [14] } & 1998 & Retrospective & 115 & $15(13.0 \%)$ & Symptomatic & $\begin{array}{l}\text { Heparin flush } \\
\text { twice weekly }\end{array}$ \\
\hline & & Prospective & 108 & $5(4.6 \%)$ & Symptomatic & $\begin{array}{l}\text { Heparin flush and warfarin } 1 \\
\mathrm{mg} / \text { day }\end{array}$ \\
\hline $\begin{array}{l}\text { Ratcliffe et al } \\
\text { [99] }\end{array}$ & 1999 & Prospective & 23 & $2(8.7 \%)$ & Symptomatic & Daily heparin-saline flushes \\
\hline $\begin{array}{l}\text { Nouwen et al } \\
{[53]}\end{array}$ & 1999 & Prospective & 48 & $7(14.6 \%)$ & Symptomatic & Not mentioned \\
\hline \multirow[t]{3}{*}{ Lagro et al [13] } & 2000 & Retrospective & 163 & $10(6.1 \%)$ & Symptomatic & $\begin{array}{l}\text { Dailly heparin-saline } \\
\text { flushes/no prophylaxis }\end{array}$ \\
\hline & & & 125 & $10(8.0 \%)$ & Symptomatic & 7 days nadroparin s.c. \\
\hline & & & 102 & $7(6.9 \%)$ & Symptomatic & 10 days nadroparin s.c. \\
\hline \multirow{2}{*}{$\begin{array}{l}\text { Fijnheer et al } \\
\text { [100] }\end{array}$} & 2002 & Prospective & 94 & $9(9.6 \%)$ & Symptomatic & Daily heparin-saline flushes \\
\hline & & & 183 & $23(12.6 \%)$ & Symptomatic & $\begin{array}{l}10 \text { days of LMWH s.c/ } 4 \\
\text { weeks of heparin }\end{array}$ \\
\hline $\begin{array}{l}\text { Harter et al } \\
{[101]}\end{array}$ & 2002 & Prospective & 233 & $4(1.5 \%)$ & Symptomatic & 10.000 IE heparin i.v./day \\
\hline Male et al [27] & 2003 & Prospective & \multicolumn{2}{|c|}{ 85Children29 (34.1\%) } & (a)Symptomatic & Continuous UFH or flushes \\
\hline Lordick et al [9] & 2003 & Prospective & 43 & $13(30.2 \%)$ & (a)Symptomatic & $\begin{array}{l}\text { Continuous UFH/LMWH } \\
\text { s.c/low dose heparin }\end{array}$ \\
\hline $\begin{array}{l}\text { Cortelezzia et al } \\
{[21]}\end{array}$ & 2003 & Retrospective & 137 & 14 (10.2\%) & Symptomatic & $\begin{array}{l}\text { Weekly heparin flush and, } \\
\text { LMWH or UFH or none }\end{array}$ \\
\hline $\begin{array}{l}\text { Van Rooden } \\
\text { et al [29] }\end{array}$ & 2005 & Prospective & 105 & $13(12.4 \%)$ & Symptomatic & $\begin{array}{l}\text { Daily urokinase in lumina of } \\
\text { the CVC }\end{array}$ \\
\hline Abdelkefi et al & 2005 & Prospective & 102 & $10(9.8 \%)$ & (a)Symptomatic & Saline infusion \\
\hline$[12]$ & & & 102 & $2(2.0 \%)$ & (a)Symptomatic & Continuous infusion of UFH \\
\hline $\begin{array}{l}\text { Cortelezzi et al } \\
\text { [5] }\end{array}$ & 2005 & Prospective & 458 & $35(7.6 \%)$ & Symptomatic & $\begin{array}{l}\text { Not mentioned } \\
14.2 \% \text { antithrombotic } \\
\text { prophylaxis }\end{array}$ \\
\hline $\begin{array}{l}\text { Magagnoli et al } \\
\text { [38] }\end{array}$ & 2006 & Retrospective & 254 & $3(1.2 \%)$ & Symptomatic & $1 \mathrm{mg}$ of warfarin daily \\
\hline \multirow[t]{2}{*}{ Niers et al [36] } & 2007 & Prospective & 41 & $7(17 \%)$ & (a)symptomatic & Nadroparin \\
\hline & & & 46 & $4(9 \%)$ & (a)symptomatic & None \\
\hline $\begin{array}{l}\text { Worth et al } \\
\text { [102] }\end{array}$ & 2009 & Prospective & 106 & $16(15.1 \%)$ & Symptomatic & Not mentioned \\
\hline $\begin{array}{l}\text { Stoffel et al } \\
\text { [103] }\end{array}$ & 2014 & Retrospective & 300 & $15(5 \%)$ & symptomatic & Not mentioned \\
\hline $\begin{array}{l}\text { Del Principe et } \\
\text { al [84] }\end{array}$ & 2013 & Retrospective & 106 & $19(18 \%)$ & Symptomatic & None/ LMWH s.c \\
\hline Joks et al [104] & 2014 & Prospective & 200 & $18(9 \%)$ & Symptomatic & Enoxaparin \\
\hline $\begin{array}{l}\text { Morano et al } \\
{[105]}\end{array}$ & 2014 & Retrospective & 1102 & $29(2.6 \%)$ & Symptomatic & Not mentioned \\
\hline
\end{tabular}

$\mathrm{UFH}=$ unfractioned heparin

$\mathrm{LMWH}=$ low molecular weight heparin 
Symptomatic CVC-related thrombosis is defined as thrombosis objectified by diagnostic imaging upon overt symptoms and signs and is found in $1.2 \%-18.0 \%$ of patients with a hematological malignancy (see table 1).

The observed incidences of CVC-related thrombosis vary considerably among the different studies, reflecting differences in types of CVCs, study design and duration, study population and various sensitivity of the examination procedures.

The incidence of CVC-related thrombosis seems to be higher in older studies compared to more recent studies which may be explained by improvements in CVC insertion technique and/or improvements in the biocompatibility of the CVCs. Currently used CVCs are composed of silicon or polyurethane and are less often associated with local thrombosis than the CVCs made of polyethylene [11].

Symptoms of CVC-related thrombosis vary widely and consist of swelling or pain of $\mathrm{arm} / \mathrm{neck} /$ head, headache, numbness of the extremity, erythema of the extremity, phlegmasia, venous distension and jaw pain. The median number of days between line insertion and CVC-related thrombosis is 16-23 days [5, 8, 12-14].

CVC-related thrombosis may have serious complications. The catheter is located deep in the mediastinum and thrombosis may be clinically silent until late in its course.

Pulmonary embolism occurs in 15\%-25\% of patients with a symptomatic CVC-related thrombosis $[15,16]$, postphlebitic syndrome arises in $14.8 \%$ of patients with an upperextremity deep vein thrombosis [17] and there is an increased risk of CVC related infections $[9,10,18]$.

\section{Pathogenesis and risk factors for CVC-related thrombosis}

The pathogenesis of CVC-related thrombosis is multifactorial. Vessel damage, a major component of Virchow's triad plays an important role. Vessel damage can be caused by a variety of factors such as mechanical injury of the venous endothelium by the catheter during the insertion, the number of vein punctures [19] and irritation of the vessel wall by chemotherapeutic agents [20, 21].

After the insertion of a CVC, a fibrin sheath can form around the CVC. The development of a CVC-related sleeve has been reported to occur in up to $47 \%$ of patients with CVCs $[22,23]$. The catheter sleeve is an adherent coating of fibrin and collagen that may envelope the CVC. This fibrin sleeve is in itself a benign complication, but can cause catheter malfunction, facilitates the development of infection and may lead to mural thrombosis.

There are several other risk factors for CVC-related thrombosis, such as CVC biocompatibility, catheter tip position, side of insertion, puncture site of insertion, inherited and acquired thrombophilic abnormalities and CVC-related infections. The position of the catheter tip in the vascular system is an important risk factor for the development of CVC-related thrombosis. The incidence of CVC-related thrombosis is 
higher in patients in whom the catheter tip is placed in the innominate vein or proximal superior vena cava as compared with the distal superior vena cava/right atrial junction $[24,25]$. Tesselaar et al showed a 2.6-fold higher risk when the catheter was located in the superior vena cava compared with the right atrium [26].

The side of insertion and puncture site of insertion are other important risk factors. Tesselaar et al showed left-sided placement to be associated with a 3.5-fold increased risk for CVC-related thrombosis compared to right-sided placement [26]. In children with acute lymphoblastic leukemia, left-sided CVCs were also associated with an increased incidence of CVC-related thrombosis. Patients with a CVC in the subclavian vein had a 44\% (22 of 50) incidence of CVC-related thrombosis compared with a 20\% (7 of 35) incidence in patients with a jugular vein CVC ( $p=0.025)$ [27]. A possible explanation for this all lies in the anatomy of the upper body venous system. In comparison with the right side, the left brachiocephalic vein is longer and has a more horizontal course, leading to a sharper angle into the superior vena cava. Compared with jugular CVCs, subclavian CVCs follow an even sharper curve into the central venous system. The CVC enters where the vein passes between the clavicle and the first rib, which may cause vein compression and kinking of the CVC [27].

There is no clear consensus regarding the role of either inherited or acquired thrombophilic states in the pathogenesis of CVC-related thrombosis [28].

CVC-related infections contribute to the pathogenesis of thromboembolic complications $[18,29,30]$. Van Rooden et al reported in a prospective study in a population of hematological patients an increased risk of CVC-related thrombosis in patients with a CVC-related infection compared with those without infection (relative risk 17.6; 95\% Cl 4.1-74.1) [29].

\section{Diagnosis of CVC-related thrombosis}

In patients with suspected CVC-related thrombosis, compression ultrasound or contrast venography are performed most often. In contrast to the numerous studies in suspected venous thrombosis of the legs, there is a paucity of diagnostic studies in upper limb thrombosis to support the efficacy of this modality.

Contrast venography was always considered to be the gold standard in detecting upper limb-venous thrombosis (UL-VT). Routine application, however, is limited due to its invasive nature and the use of a contrast medium.

Compression ultrasound (CUS) with Doppler and color imaging is easier applicable for the detection of thrombosis and is therefore more widely used. However, the anatomy of the arm veins, especially the part of the subclavian vein that crosses the clavicle, makes a proper diagnosis with ultrasound sometimes difficult.

In a systematic review of studies on the diagnosis of suspected UL-VT a sensitivity of CUS ranging from $56 \%$ to $100 \%$ and a specificity ranging from $94 \%$ to $100 \%$ has been reported [31]. 
Clinical probability scores and D-dimer tests are commonly used as safe diagnostic tests in the diagnostic workup for thrombosis of the lower limbs. Only limited data are available on the applicability of these diagnostic tools for upper limb venous thrombosis. A study attempted to develop a clinical score combining 4 items: 1) presence of a CVC or pacemaker, 2) localized pain, 3) unilateral pitting edema, and 4) other diagnosis as possible. Based on this score 214 patients were divided into a low-, intermediate-, or a high- probability group. The prevalence of ultrasonography confirmed upper limb venous thrombosis in the 3 groups was, respectively $12 \%, 20 \%$, and $70 \%$. The sensitivity of this score was $78 \%$ with a specificity of $64 \%$ [32]. A prospective study in 52 patients evaluated the accuracy of the D-dimer test. The sensitivity, specificity, and positive and negative predictive values of the test were $100 \%, 14 \%, 32 \%$ and $100 \%$ respectively [33]. Overall, the safety of withholding anticoagulant therapy based on a low clinical probability score and a normal D-dimer has not been evaluated in upper limb venous thrombosis.

The most recent ACCP guidelines suggest the use of a Doppler or color Doppler ultrasound over other initial tests such a venography [34]. If the ultrasound is negative in a patient with a high clinical suspicion of CVC-related thrombosis, a contrast venography should be performed.

\section{Is antithrombotic prophylaxis indicated?}

The efficacy and safety of using systemic anticoagulant prophylaxis for prevention of catheter-associated thrombosis in patients with hematological malignancies in the form of fixed dose warfarin, adjusted dose warfarin, low molecular weight heparin (LMWH) and unfractionated heparin has been examined in several randomized, controlled trials [35-37].

The earliest studies suggested that low dose warfarin or LMWHs could significantly reduce the incidence of CVC-related thrombosis [14, 20, 38]. However, these were small, open label, often retrospective studies often using asymptomatic CVC-related thrombosis as an endpoint.

In a well-conducted, randomized, double blind trial, Couban et al found that the administration of warfarin $1 \mathrm{mg}$ daily did not reduce the incidence of symptomatic CVCrelated thrombosis in a patient population with mostly hematological malignancies [37]. Major limitations of their study were the unexpectedly low incidence of thrombosis (only 11 symptomatic CVC-related thrombosis in 255 patients) and the timing of starting warfarin (study drug was always administered after CVC insertion and may have been started up to 72 hours later). The WARP trial, a multicenter, open label, randomized trial comparing no warfarin, fixed dose warfarin and dose adjusted warfarin included 1590 cancer patients with central venous catheters. Fixed dose warfarin did not reduce the incidence of symptomatic CVC-related thrombosis compared to no treatment (7\% 
versus $6 \%, p=0.98)$. Dose adjusted warfarin did significantly reduce the incidence of CVC-related thrombosis $(2.7 \%$ versus $7.2 \%, R R \quad 0.38, p=0.002)$ at the expense of increased major bleeding complications (3.4\% versus $1.5 \%, p=0.04$ ). Most of the patients included had a solid tumor [35].

Similarly, several randomized studies explored the efficacy of prophylactic LMWHs. Niers et al performed a single-center, prospective, randomized, placebo-controlled double blind trial to evaluate the efficacy of low molecular weight heparins once daily for the prevention of CVC-related thrombosis. They found no beneficial effect [36].

Lagro et al found no effect using prophylactic nadroparin on the incidence of CVCrelated thrombosis in 382 patients receiving a stem cell transplantation [13]. Their study however, has a retrospective design and the patients only received nadroparin the first 6-10 days after insertion of the CVC. Since CVC-related thrombosis is observed at a median of 16-23 days after insertion of the CVC the time course of nadroparin administration may have been too short.

The only study showing a significant reduction in CVC-related thrombosis (and infections) used continuous intravenous unfractionated heparin (UFH) in 108 adults and children undergoing bone marrow transplantation. CVC-related thrombosis occurred in $1.5 \%$ of the CVCs inserted in patients of the heparin group, and in $12.6 \%$ of the control group ( $P=0.03$ ). Two patients in the heparin group and three patients in the control group experienced severe bleeding $(P=0.18)$ [39]. The major limitations of this study is its small sample size. No studies have been performed to confirm these results.

Based on above mentioned studies the ACCP guidelines and the International clinical practice guidelines for the treatment and prophylaxis of thrombosis associated with central venous catheters in patients with cancer recommend against the routine administration of pharmacologic prophylaxis to prevent CVC-related thrombosis [7, 40, 41]. There is no clear consensus regarding the role of either inherited or acquired thrombophilic states in the pathogenesis of CVC-related thrombosis [28], nor is there a clear recommendation on the use of prophylactic measures in this population [41].

\section{Treatment of CVC-related thrombosis}

The aims of treatment of CVC-related thrombosis are to reduce the mortality and morbidity from the acute event and to reduce late complications.

The management of patients who develop a CVC-related thrombosis is not standardized. Treatment strategies consist of thrombolytic therapy, initiation of systemic anticoagulation, removal of the catheter or both.

In non-hematological patients with a CVC-related thrombosis the preferred treatment is the combination of a LMWH followed by oral anticoagulation for at least 36 months $[42,43]$. No prospective, randomized studies have been published on this subject. 
The management of thrombosis in hematological and often thrombocytopenic and coagulopathic patients is challenging because low molecular weight heparins and oral anticoagulants are relatively contraindicated because of the high risk of major bleeding [44]. Many hematological patients who undergo line removal still require central venous access and insertion of another CVC further increases the thrombotic risk.

There is only limited information on the treatment of CVC-related thrombosis in hematological patients. No randomized trials have been published on this subject in the literature. In a retrospective study the treatment and outcome of 112 cancer patients with a CVC-related thrombosis was reported. Treatment consisted of anticoagulation $(n=39)$, anticoagulation with CVC removal or replacement $(n=22)$, other therapy $(n=7)$ or no therapy $(n=8)$. Regardless of the intervention no patients had a major adverse outcome like pulmonary emboli, vascular comprise of a limb, or death. Only 4 patients did not have resolution of their presenting symptoms, all of whom were treated with line replacement [45].

Kovacs et al assessed in a prospective cohort study the safety and effectiveness of a management strategy for CVC-related thrombosis in cancer patients consisting of dalteparin and warfarin without the need for removal of the CVC. There were no episodes of recurrent venous thrombosis and $3(4 \%)$ major bleeds in 74 cancer patients. No lines were removed due to infusion failure or recurrence/extension of thrombosis [46]. Oliver et al retrospectively compared outcomes of patients with acute leukemia and a CVC-related thrombosis who were treated or not with anticoagulation. They found no differences in bleeding [44].

Whether a CVC-related thrombosis necessitates the removal of the CVC remains unclear. The decision of removal is usually left to the discretion of the attending physician. The decision to remove a central venous catheter after a thrombotic event must be balanced against other factors, such as difficulty of venous access, need for further treatments and blood sampling, and patient preference [19]. If removal of the CVC is necessary, this may be performed after 3-5 days of anticoagulant treatment, although the optimal timing is unclear [40]. The ACCP guidelines, American Society of Clinical Oncology clinical practice guidelines and the International clinical practice guidelines for CVC-related thrombosis in cancer patients from Debourdeau are consistent with this approach, and do not recommend removal of a CVC if the device is still functioning and there is an ongoing need for the CVC [7, 41, 47].

No randomized comparison of thrombolytic therapy with heparin has been performed in hematological or non-hematological patients with a CVC-related thrombosis. In a retrospective, nonrandomized analysis of 95 patients with a subclavian vein thrombosis systemic thrombolysis was compared with anticoagulant therapy. Systemic thrombolysis was associated with an acceptable primary technical success rate, but a rather high frequency of bleeding complications (21\% versus none in the group of anticoagulants only) [48] . 
The conventional therapy for a blocked CVC resulting from catheter tip occlusion or catheter sleeve occlusion is local thrombolytic therapy with a low dose of single or repeated bolus of urokinase, streptokinase or tissue plasminogen activator. This therapy restores catheter patency in most patients, provided the CVC is well positioned $[49,50]$.

There is no consensus regarding the treatment of asymptomatic thrombosis. Since unrecognized thrombosis may be clinically important [10] further prospective studies are needed to determine if the treatment of asymptomatic thrombosis will prevent occlusion of the CVC or CVC-related infections.

\section{CVC-RELATED INFECTIONS}

CVC-related infections can be divided into catheter colonization, exit-site infections, tunnel infections and blood stream infections [51].

The prevalence of CVC-related bloodstream infections in hematological patients varies from $0.0 \%$ to $40.6 \%$, depending on the patient and device characteristics and the definition used for a CVC-related bloodstream infection (see table 2). Only a minority of these publications adhere to the U.S Hospital Infection Control Practices Advisory Committee (HICPAC) definitions for intravascular device-related infections [52].

The risk of infection is particularly high in neutropenic patients, patients undergoing myeloablative chemotherapy followed by autologous stem cell transplantation or patients with a CVC-related thrombosis $[9,53]$.

\section{Pathogenesis and etiology of CVC-related infections}

Following catheter insertion, a thrombin layer or sheath covers the external and the internal surfaces of the intravascular segment. This sheath, which is rich in host-derived proteins, such as fibrin, fibronectin, thrombospondin, and laminin, promotes adherence of potential microbial pathogens to the surface. Furthermore, Staphylococci, Candida and some other microbes produce a slimy material rich in exopolysaccharides, resulting in the formation of a microbial biofilm. This biofilm helps these organisms adhere to and survive on the surfaces of foreign bodies in the bloodstream [54].

The organisms causing a CVC-related infection can gain access to the device through four different routes: invasion of the skin insertion site, contamination of the catheter hub, hematogenous spread from a distant site of infection or infusion of contaminated fluid through the device $[51,55]$.

For long-term (>2 weeks in situ) CVCs, contamination of the catheter hub is the most common route of infection. From the contaminated hub, the organisms migrate along the internal surface of the CVC, where they create a bloodstream infection. For 
short term, non-tunneled, catheters, skin contamination is the most likely route of infection, whereby organisms migrate along the external surface of the CVC and the intercutaneous and subcutaneous segments, leading to colonization of the intravascular catheter tip, which may lead to blood stream infection[54, 55]

Table 2. Incidence of CVC-related bloodstream infections in hematological patients

\begin{tabular}{|c|c|c|c|c|}
\hline Author & Year & Study design & No CVC & CVC-related bacteremia \\
\hline Carratala et al [106] & 1999 & Prospective & $\begin{array}{l}57 \text { heparin lock } \\
60 \text { heparin-vancomycin lock }\end{array}$ & $\begin{array}{l}4(7.0 \%) \\
0(0 \%)\end{array}$ \\
\hline Nouwen et al [53] & 1999 & Prospective & 48 & $10(20.8 \%)$ \\
\hline Lagro et al [13] & 2000 & Retrospective & 390 & $46(11.8 \%)$ \\
\hline Harter et al [101] & 2002 & Prospective & $\begin{array}{l}113 \text { standard CVCs } \\
120 \text { silver-coated CVCs }\end{array}$ & $\begin{array}{l}10(8.8 \%) \\
6(5 \%)\end{array}$ \\
\hline Karthaus et al [107] & 2002 & Prospective & 178 & $17(9.6 \%)$ \\
\hline Cortelezzia et al [21] & 2003 & Retrospective & 150 also PICCS & $16(10.6 \%)$ \\
\hline Lordick et al [9] & 2003 & Prospective & 43 & $4(9.3 \%)$ \\
\hline Nosari et al [108] & 2004 & Retrospective & 227 & $23(10.1 \%)$ \\
\hline Abdelkefi et al [12] & 2005 & Prospective & $\begin{array}{l}102 \text { (heparin group) } \\
102 \text { (control group) }\end{array}$ & $\begin{array}{l}7(6.8 \%) \\
17(16.6 \%)\end{array}$ \\
\hline Cortelezzi et al [5] & 2005 & Prospective & 458 & $21(4.6 \%)$ \\
\hline Jaeger et al [76] & 2005 & Prospective & $\begin{array}{l}55 \text { standard CVCs } \\
55 \mathrm{CH}-\mathrm{SS} \text { CVCs }\end{array}$ & $\begin{array}{l}8(14.5 \%) \\
1(1.8 \%)\end{array}$ \\
\hline Van Rooden et al [29] & 2005 & Prospective & 105 & $14(13.3 \%)$ \\
\hline Niers et al [36] & 2007 & Prospective & $\begin{array}{l}\text { 56; nadroparine prophylaxis } \\
57\end{array}$ & $\begin{array}{l}9(16 \%) \\
10(17.5 \%)\end{array}$ \\
\hline Van Rooden et al [92] & 2008 & prospective & $\begin{array}{l}82 ; \text { urokinase lock } \\
78\end{array}$ & $\begin{array}{l}6(7.3 \%) \\
14(17.9 \%)\end{array}$ \\
\hline Worth et al [102] & 2009 & Prospective & 106 (75 PICCS) & $18(17 \%)$ \\
\hline Slobbe et al [95] & 2010 & Prospective & 448 & 45 (10\%) \\
\hline Del Principe et al [83] & 2013 & Retrospective & 106 & $43(40.6 \%)$ \\
\hline Morano et al [105] & 2014 & Retrospective & 1102 & $138(12.5 \%)$ \\
\hline Sakai et al [109] & 2014 & Retrospective & 148 & 22 (14.9\%) \\
\hline
\end{tabular}

PICCS: peripherally inserted central venous catheters

CH-SS CVC: chlorhexidine and silver sulfadiazine-impregnated central venous catheter

CVC-related infections in hematological patients are usually caused by coagulasenegative Staphylococcus (62.5\%), Staphylococcus aureus (4.2\%), gram negative bacilli like Enterobacteriaceae, Escherichia coli and Pseudomonas species (29\%) or Candida species (4.2\%) [12].

In hematological patients undergoing intensive chemotherapy alternative sources of bacteremia like microbial translocation during chemotherapy-induced mucositis are 
important as well. The CDC (Centers for disease control and prevention) recently developed a new definition termed "mucosal barrier injury-laboratory-confirmed bloodstream infection". It applies to bacteremic patients with central lines who could have a gastrointestinal source for the bacteremia associated with mucosal injury related to post chemotherapy neutropenia or graft-versus-host disease [4].

\section{Diagnosis of CVC-related infections}

Diagnosis of CVC-related infections is notoriously difficult and challenging. The clinical presentation of CVC-related infections consists of non-specific systemic manifestations and local manifestations at the CVC insertion site and may be subtle or absent in neutropenic cancer patients [4]. It may be difficult to discriminate between infection and contamination. Clinical studies are hampered by the lack of a gold standard for the diagnosis.

Clinical signs often are unreliable to indicate the presence of a catheter-related infection [56, 57]. Microbiological criteria are therefore essential.

The most common techniques based on removal of the CVC are Maki's semiquantitative culture and quantitative endoluminal cultures. Quantitative cultures of the catheter segment requires either flushing the segment with broth, or vortexing, or sonication it in broth, followed by serial dilutions and surface plating on blood agar [5860]. The most widely used method is the semiquantitative method, in which the catheter segment is rolled across the surface of an agar plate and colony-forming units are counted after overnight incubation [61].

The major disadvantages of this roll plate method are that only the external surface of the CVC is cultured and the need for removal of the catheter. Since these catheters are often integral to patient care in hematological patients, this often requires reinsertion and exposing patients again to all risks related to this procedure. The semiquantitative roll plate method is of limited usefulness in hematological patients with mostly long-term catheters, in which the internal surface is the predominant source of colonization and bloodstream infection [55].

Diagnostic methods in which the CVC is left in situ include paired quantitative blood cultures, paired qualitative blood cultures with a continuously monitored differential time to positivity or the endoluminal brush $[51,54,62]$. Quantitative blood cultures drawn through the CVC and concomitantly by venapuncture from a peripheral vein are time consuming and expensive. If the CVC is infected the blood drawn through it shows a 5 -fold increase in the concentration of organisms compared with the blood drawn percutaneously from a peripheral vein. Safdar et al found in their meta-analysis a sensitivity of $87 \%$ and a specificity of $98 \%$ and concluded that paired quantitative blood cultures is the most accurate diagnostic method in patients with long-term CVCs [63].

The differential time to positivity (DTP) of paired CVC and peripheral blood cultures provides comparable sensitivity and acceptable specificity ( $85 \%$ and $81 \%$ respectively), 
at no increased costs [63]. Abdelkefi et al showed that DTP is useful for the diagnosis of catheter-related bloodstream infection in hematopoietic stem cell transplant recipients (86\% sensitivity and $87 \%$ specificity) [64].

Both culture techniques require a blood sample to be drawn through the CVC lumen, but in $15-50 \%$ of cases this may not be achieved $[62,65]$. The endoluminal brush has no such limitation and has a sensitivity of $100 \%$ and a specificity of $89 \%$ [62]. It is theoretically possible to induce a peripheral bacteremia by endoluminal brushing of colonized CVCs. Dobbins et al found no peripheral bacteremia either 1 minute or 1 hour post-brushing in any of eight cases with significant endoluminal colonization [66]. Few studies have assessed the endoluminal brush and most of the studies identified were performed by the same group of investigators. Since important side-effects theoretically may occur, it is not a method that can be recommended without further study.

\section{Prevention of CVC-related infections}

The introduction of a bundle of aseptic techniques-such as barrier precautions, application of chlorhexidine during insertion, hand hygiene, avoidance of femoral sites, and removal of the CVC when it is not needed-was shown to decrease the risk of CVCrelated infections in short term catheters (dwell time of $<2$ weeks), which are used mostly in critical care patients $[67,68]$.

These measures have not been fully studied in hematological patients who have their CVC in situ for more than 2 weeks, have intraluminal biofilm colonization and often have a gastrointestinal source for the bacteremia. These are unlikely to be prevented by only the aseptic bundle measures applied during insertion which makes further interventions required.

Any attempt to reduce the routine number of CVC manipulations at any site could reduce the risk of CVC-related infections. Good training in catheter manipulations is probably one of the best methods for guarding against CVC-related bacteremia. The presence of a specialized team of nurses committed to the care of CVCs or the knowledge of proper maintenance procedure techniques shared by all nurses are both effective approaches for the reduction of CVC-related infection incidence in hospitalized patients $[52,69]$.

Continuing quality improvement programs to assure compliance with catheter care guidelines significantly reduce primary bloodstream infection $[52,69]$.

Moller et all showed a reduction of more than $50 \%$ in the incidence rate of CVCrelated infections with a systematic, individualized, supervised patient education program and self-care for the catheter [70].

To prevent CVC-related infections, antibiotic lock prophylaxis has been attempted by flushing and filling the lumen of the CVC with an antibiotic solution and leaving the solution to dwell in the lumen of the CVC. In 126 immunocompromised children the use 
of either vancomycin/ciprofloxacin/heparin (VCH) or vancomycin/heparin (VH) compared to heparin alone significantly increased the time to develop a CVC-related infection and decreased the amount of gram-positive CVC-related infections (VH, P = 0.028; VHC, $P=0.022$ ) [71]. However, the Centers for Disease Control and Prevention guidelines recommend against prophylactic use of vancomycin because it is an independent risk factor for acquisition of vancomycin-resistant enterococci [72].

Systemic antibiotic prophylaxis at the time of CVC insertion in 65 neutropenic patients has not been shown to reduce the incidence of CVC-related bloodstream infection [73]. In another study a single dose of teicoplanin did decrease the risk of gram-positive CVC-related infections in neutropenic patients. The benefit of prophylactic teicoplanin was observed particularly among patients who were already neutropenic at the time of catheterization [74]. In the study of Ljungman only 2 patients were neutropenic at the time of CVC insertion which is a possible explanation for the observed difference [73].

Antiseptic-impregnated CVCs are effective in preventing CVC-related infections. In a meta-analysis Veenstra et al showed that short-term (less than two weeks) use of CVCs impregnated with chlorhexidine/silver sulfadiazine reduced the risk of CVC-related bloodstream infections by about 40\% [75]. This meta-analysis, however, was not limited to only hematological patients.

In a prospective, randomized trial in 106 patients with severe neutropenia, CVCrelated colonization and $\mathrm{CVC}$-related bloodstream infections were observed less frequently in the study group with chlorhexidine and silver sulfadiazine impregnated CVCs compared to the control group using a standard uncoated CVC. The CVCS, however, were only in situ for a period of two weeks which is a relatively short period of time for hematological patients [76]. Logghe et al showed that the use of antiseptic impregnated CVCs in hematological patients reduced neither the overall risk of CVCrelated bloodstream infection, nor the CVC-related infection rate, nor the delay for the occurrence of infections. The CVCs were in situ for a mean of 20 days (standard deviation 12 days) [77]. This finding probably reflects reduced antimicrobial activity of the CVC over time and a lack of protection from microbes invading the luminal surface of the CVC from contaminated hubs [77].

Significant controversy surrounds the usefulness of CVCs impregnated with antimicrobial agents for the prevention of CVC-related bloodstream infections [78-80]. McConell et al reviewed 11 trials of antimicrobial impregnated CVCs versus uncoated CVCs and they concluded that there is a lack of solid evidence to support a benefit of antimicrobial impregnated CVCs in reducing the rate of CVC-related bloodstream infections [80]. Others assert that there is a large body of evidence that demonstrates a powerful decrease in the risk of infection [78, 79].

In a meta-analysis Falagas et al demonstrated that CVCs impregnated with rifampicin and monocyclin are safe and efficacious in reducing the rate of catheter 
colonization and CVC-related bloodstream infections [81]. Most included patients in all reviews however were not hematological patients.

Hanna et al performed a prospective study in hemato-oncological patients with 356 CVCs who were randomized between non-impregnated CVCs and CVCs impregnated with minocycline/rifampin. 14 CVC-related bloodstream infections occurred in the nonimpregnated CVCs and only 3 occurred in the group with CVCs impregnated with minocycline/rifampin ( $8 \%$ versus $1.6 \% ; P=0.003$ ). The mean duration of catheterization was respectively $63.01 \pm 30.88$ days and $66.21 \pm 30.88$ days [82].

\section{Treatment of CVC-related infections}

Patients with a CVC-related infection should be separated in those with complicated infections, in which there is septic thrombosis, endocarditis, osteomyelitis or possible metastatic seeding, and those with uncomplicated CVC-related infections in which there is no evidence of such complications. Patients with an uncomplicated CVC-related infection should receive 10-14 days of antimicrobial therapy. Relapse, continuous fever, or bacteremia, despite removal of the catheter is consistent with the suspicion of a persistent focus of infection. This implies prolonged (4-6 weeks) or modified antimicrobial treatment and an active search for metastatic abscess, septic thrombophlebitis, or endocarditis.

Because removal of a CVC is often a management challenge, it is important to be sure that one is dealing with a true CVC-related bloodstream infection, rather than skin contamination, catheter colonization, or infections from another source.

The CVC may be left in situ in the presence of an uncomplicated infection due to coagulase-negative staphylococci. Removal is mandatory in severe or complicated infections such as shock, persistent fever, persistent bacteremia, or bacteremia with certain micro-organisms (S. aureus, gram-negative bacilli, Candida spp) $[4,51,83]$.

\section{RELATION BETWEEN CVC-RELATED INFECTIONS AND THROMBOSIS}

There is accumulating evidence showing that CVC-related thrombosis and infections are interrelated and can therefore not be seen as separate entities. There seems to be a bidirectional relationship [9, 29, 30, 84].

A major contributing factor in both CVC-related thrombosis and CVC-related infections might be fibrin sheath formation around the external portion of the catheter and within the catheter lumen [85].

The composition of CVC-related thrombi consists of several proteins such as fibrin, fibronectin, collagen, laminin, and several types of immunoglobulins. Micro-organisms, especially S. aureus and S.epidermidis, easily adhere to thrombin sheaths $[85,86]$. 
These micro-organisms are able to produce a coagulase enzyme that enhances the thrombogenic process.

There is sufficient evidence that thrombosis affects inflammation in more ways. Activated coagulation proteases interact with protease-activated receptors which are believed to play a role in translating coagulation products in inflammatory signals. Thrombin induces up-regulation of various proinflammatory cytokines in vitro [87-89].

Lordick et al detected a CVC-related infection in 14 of 43 hemato-oncological patients. In 12 of the 14 patients with a CVC-related infection a CVC-related thrombosis preceded the CVC-related infection [9] .

After an episode of CVC-related infection, the risk of clinically manifest thrombosis is markedly increased. In a group of patients with a CVC-related infection, the frequency of subsequent clinically manifest thrombosis was 44\% (11 of 25 patients), compared with $3 \%$ thrombosis in the patients without CVC-related infection ( 2 of 80 patients). This yields a relative risk of 17.6. The absolute risk of developing a clinically manifest thrombosis increased with the severity of infection; a 57\% thrombosis risk was observed after an episode of CVC-related bloodstream infection, versus $27 \%$ in patients with a positive lock fluid culture [29]. Del Principe et al found the occurrence of CVC-related thrombosis to be associated with CVC-exit site infections and sepsis [84].

CVC-related infections might induce an inflammatory response which can result in activation of coagulation, due to tissue factor mediated thrombin generation, downregulation of physiological anticoagulant mechanisms and inhibition of fibrinolysis. This could induce thrombus formation which may further promote catheter colonization, bacterial biofilm growth and eventually bloodstream seeding $[88,89]$.

As described above fibrin sheath formation around the external portion of the CVC and within the catheter lumen has been implicated as a major contributing factor in both occlusive and infectious events [85]. Interventions designed to decrease fibrin deposition and thrombus formation have the potential to reduce CVC-related infections. In a randomized study the use of a continuous infusion of low-dose unfractionated heparin (100U/kg per day) was investigated to prevent CVC-related thrombosis and CVC-related bloodstream infections in patients with hematological diseases. CVC-related bloodstream infection occurred in 6.8\% (7 of 102 CVCs) of those in the heparin group and in $16.6 \%$ (17 of $102 \mathrm{CVCS}$ ) of those in the control group ( $\mathrm{P}=$ 0.03). CVC-related thrombosis was observed more frequently in the control group (10 of 102) than in the heparin group (2 of 102)( $P=0.017)$. Four patients experienced severe bleeding in the heparin group and five patients experienced severe bleeding in the control group $(P=0.2)[12]$.

Considerable uncertainty persists about the optimum choice of 'locking protocol' of the CVC [90]. Locking of the catheter lumina with an anticoagulant is performed to decrease 
fibrin deposition, maintain patency of the CVC and to avoid catheter clotting and CVCrelated thrombosis and infections. The most widely used locking solution in hematological patients undergoing intensive chemotherapy is heparin [90]. Since heparin has side effects like unintentional systemic anticoagulation, interference with lab assessments of clotting, heparin-induced thrombocytopenia and enhances staphylococcal biofilm formation alternatives to heparin are studied [91].

Locking the CVC with urokinase in hematological patients undergoing intensive chemotherapy decreases fibrin deposition and thrombus formation and in this way decreases the risk of CVC-related bloodstream infections and thrombosis $[92,93]$.

In a prospective randomized trial in pediatric patients undergoing chemotherapy or bone marrow transplantation the use of taurolidine citrate lock solution was associated with significantly fewer CVC-related bloodstream infections. There were similar rates of thrombotic complications in both groups [94].

Slobbe et al studied the efficacy and safety of a daily 70\%-ethanol lock on the prevention of CVC-related BSI in hematological patients with long-term tunneled catheters. They found a non-significant reduction of $41 \%$ for patients treated with ethanol locks [95].

Trisodium citrate (TSC) prevents CVC-related thrombosis and infections via local anticoagulation by chelating ionized calcium. TSC exerts a broad antimicrobial and antiyeast effect [96]. TSC has been shown to be an effective antimicrobial catheter locking solution in hemodialysis patients. TSC lowered the incidence of CVC-related bloodstream infections in hemodialysis patients from 4.1 CVC-related BSI per 1000 catheter days to 1.1 per 1000 catheter days [97]. Therefore a randomized controlled trial was performed to determine the efficacy of TSC as a locking solution compared to heparin in preventing CVC-related infections and thrombosis in patients with hematological malignancies undergoing intensive chemotherapy. No differences in CVCBSI with gram positives and/or CVC-related thrombosis were seen. A statistically significant reduction of CVC-BSI with gram negative rods in the citrate group was seen [98].

\section{CONCLUSIONS}

CVC-related thrombosis and infections are frequently occurring complications and may cause significant morbidity in patients with hematological malignancies. CVC-related thrombosis and infections are related and can therefore not be seen as separate entities.

There is need for a specific approach regarding diagnosis and treatment of CVCrelated thrombosis and infections with specific attention for the preservation of the catheter. 


\section{CHAPTER 2}

It is clear that the prevention of CVC-related infections and thrombosis is of utmost importance and will help to decrease patients suffering as well as costs of patient management. Since the fibrin sleeve has been implicated as a major contributing factor in both CVC-related thrombosis and CVC-related infections, interventions designed to decrease fibrin deposition may be the future.

Since data on CVC-related infections and thrombosis in hematological patients have been obtained mainly from retrospective studies of small sample size, prospective, randomized studies of prophylactic measures concerning CVC-related thrombosis and infections are warranted. Many topics remain unsolved and there is still an urgent need for effective, cheap and easy to implement preventive measures. 


\section{REFERENCES}

1. McGee DC, Gould MK. Preventing complications of central venous catheterization. N Engl J Med 2003; 348: 1123-1133.

2. Jarvis WR. Selected aspects of the socioeconomic impact of nosocomial infections: morbidity, mortality, cost, and prevention. Infect Control Hosp Epidemiol 1996; 17: 552-557.

3. Pittet D, Tarara D, Wenzel RP. Nosocomial bloodstream infection in critically ill patients. Excess length of stay, extra costs, and attributable mortality. Jama 1994; 271: 1598-1601.

4. Raad I, Chaftari AM. Advances in prevention and management of central line-associated bloodstream infections in patients with cancer. Clin Infect Dis 2014; 59 Suppl 5: S340-343.

5. Cortelezzi A, Moia M, Falanga $A$ et al. Incidence of thrombotic complications in patients with haematological malignancies with central venous catheters: a prospective multicentre study. $\mathrm{Br} \mathrm{J}$ Haematol 2005; 129: 811-817.

6. Rolston KV. Prediction of neutropenia. Int J Antimicrob Agents 2000; 16: 113-115.

7. Debourdeau P, Farge D, Beckers $M$ et al. International clinical practice guidelines for the treatment and prophylaxis of thrombosis associated with central venous catheters in patients with cancer. J Thromb Haemost 2013; 11: 71-80.

8. van Rooden CJ, Rosendaal FR, Barge RM et al. Central venous catheter related thrombosis in haematology patients and prediction of risk by screening with Doppler-ultrasound. Br J Haematol 2003; 123: 507-512.

9. Lordick F, Hentrich M, Decker T et al. Ultrasound screening for internal jugular vein thrombosis aids the detection of central venous catheter-related infections in patients with haemato-oncological diseases: a prospective observational study. Br J Haematol 2003; 120: 1073-1078.

10. Journeycake JM, Buchanan GR. Catheter-related deep venous thrombosis and other catheter complications in children with cancer. J Clin Oncol 2006; 24: 4575-4580.

11. Pottecher T, Forrler M, Picardat $P$ et al. Thrombogenicity of central venous catheters: prospective study of polyethylene, silicone and polyurethane catheters with phlebography or post-mortem examination. Eur J Anaesthesiol 1984; 1: 361-365.

12. Abdelkefi A, Torjman L, Ladeb S et al. Randomized trial of prevention of catheter-related bloodstream infection by continuous infusion of low-dose unfractionated heparin in patients with hematologic and oncologic disease. J Clin Oncol 2005; 23: 7864-7870.

13. Lagro SW, Verdonck LF, Borel Rinkes IH, Dekker AW. No effect of nadroparin prophylaxis in the prevention of central venous catheter (CVC)-associated thrombosis in bone marrow transplant recipients. Bone Marrow Transplant 2000; 26: 1103-1106.

14. Boraks P, Seale J, Price J et al. Prevention of central venous catheter associated thrombosis using minidose warfarin in patients with haematological malignancies. Br J Haematol 1998; 101: 483-486.

15. Monreal M, Lafoz E, Ruiz J et al. Upper-extremity deep venous thrombosis and pulmonary embolism. A prospective study. Chest 1991; 99: 280-283.

16. Monreal M, Raventos A, Lerma R et al. Pulmonary embolism in patients with upper extremity DVT associated to venous central lines--a prospective study. Thromb Haemost 1994; 72: 548-550.

17. Prandoni P, Polistena P, Bernardi E et al. Upper-extremity deep vein thrombosis. Risk factors, diagnosis, and complications. Arch Intern Med 1997; 157: 57-62.

18. Raad, II, Luna M, Khalil SA et al. The relationship between the thrombotic and infectious complications of central venous catheters. Jama 1994; 271: 1014-1016.

19. Lee $A Y$, Levine $M N$, Butler $G$ et al. Incidence, risk factors, and outcomes of catheter-related thrombosis in adult patients with cancer. J Clin Oncol 2006; 24: 1404-1408.

20. Bern MM, Lokich JJ, Wallach SR et al. Very low doses of warfarin can prevent thrombosis in central venous catheters. A randomized prospective trial. Ann Intern Med 1990; 112: 423-428.

21. Cortelezzia A, Fracchiolla NS, Maisonneuve $P$ et al. Central venous catheter-related complications in patients with hematological malignancies: a retrospective analysis of risk factors and prophylactic measures. Leuk Lymphoma 2003; 44: 1495-1501. 
22. Martin C, Viviand X, Saux P, Gouin F. Upper-extremity deep vein thrombosis after central venous catheterization via the axillary vein. Crit Care Med 1999; 27: 2626-2629.

23. Xiang DZ, Verbeken EK, Van Lommel AT et al. Composition and formation of the sleeve enveloping a central venous catheter. J Vasc Surg 1998; 28: 260-271.

24. Eastridge BJ, Lefor AT. Complications of indwelling venous access devices in cancer patients. J Clin Oncol 1995; 13: 233-238.

25. Luciani A, Clement O, Halimi P et al. Catheter-related upper extremity deep venous thrombosis in cancer patients: a prospective study based on Doppler US. Radiology 2001; 220: 655-660.

26. Tesselaar ME, Ouwerkerk J, Nooy MA et al. Risk factors for catheter-related thrombosis in cancer patients. Eur J Cancer 2004; 40: 2253-2259.

27. Male $C$, Chait $P$, Andrew $M$ et al. Central venous line-related thrombosis in children: association with central venous line location and insertion technique. Blood 2003; 101: 4273-4278.

28. Boersma RS, Hamulyak K, Cate HT, Schouten HC. Congenital thrombophilia and central venous catheterrelated thrombosis in patients with cancer. Clin Appl Thromb Hemost 2010; 16: 643-649.

29. van Rooden CJ, Schippers EF, Barge RM et al. Infectious complications of central venous catheters increase the risk of catheter-related thrombosis in hematology patients: a prospective study. J Clin Oncol 2005; 23: 2655-2660.

30. Timsit JF, Farkas JC, Boyer JM et al. Central vein catheter-related thrombosis in intensive care patients: incidence, risks factors, and relationship with catheter-related sepsis. Chest 1998; 114: 207-213.

31. Mustafa BO, Rathbun SW, Whitsett TL, Raskob GE. Sensitivity and specificity of ultrasonography in the diagnosis of upper extremity deep vein thrombosis: a systematic review. Arch Intern Med 2002; 162: 401404.

32. Constans J, Salmi LR, Sevestre-Pietri MA et al. A clinical prediction score for upper extremity deep venous thrombosis. Thromb Haemost 2008; 99: 202-207.

33. Merminod T, Pellicciotta S, Bounameaux H. Limited usefulness of D-dimer in suspected deep vein thrombosis of the upper extremities. Blood Coagul Fibrinolysis 2006; 17: 225-226.

34. Bates SM, Jaeschke R, Stevens SM et al. Diagnosis of DVT: Antithrombotic Therapy and Prevention of Thrombosis, 9th ed: American College of Chest Physicians Evidence-Based Clinical Practice Guidelines. Chest 2012; 141: e351S-418S.

35. Young AM, Billingham LJ, Begum $G$ et al. Warfarin thromboprophylaxis in cancer patients with central venous catheters (WARP): an open-label randomised trial. Lancet 2009; 373: 567-574.

36. Niers TM, Di Nisio M, Klerk CP et al. Prevention of catheter-related venous thrombosis with nadroparin in patients receiving chemotherapy for hematologic malignancies: a randomized, placebo-controlled study. J Thromb Haemost 2007; 5: 1878-1882.

37. Couban S, Goodyear M, Burnell M et al. Randomized placebo-controlled study of low-dose warfarin for the prevention of central venous catheter-associated thrombosis in patients with cancer. J Clin Oncol 2005; 23: 4063-4069.

38. Magagnoli M, Masci G, Castagna L et al. Prophylaxis of central venous catheter-related thrombosis with minidose warfarin in patients treated with high-dose chemotherapy and peripheral-blood stem-cell transplantation: retrospective analysis of 228 cancer patients. Am J Hematol 2006; 81: 1-4.

39. Abdelkefi A, Ben Othman $T$, Kammoun $L$ et al. Prevention of central venous line-related thrombosis by continuous infusion of low-dose unfractionated heparin, in patients with haemato-oncological disease. A randomized controlled trial. Thromb Haemost 2004; 92: 654-661.

40. Zwicker J, Connolly G, Carrier M et al. Catheter-associated deep vein thrombosis of the upper extremity in cancer patients: guidance from the SSC of the ISTH. J Thromb Haemost 2014; 12 (5); 796-800.

41. Schiffer CA, Mangu PB, Wade JC et al. Central venous catheter care for the patient with cancer: American Society of Clinical Oncology clinical practice guideline. J Clin Oncol 2013; 31: 1357-1370.

42. Buller HR, Agnelli G, Hull RD et al. Antithrombotic therapy for venous thromboembolic disease: the Seventh ACCP Conference on Antithrombotic and Thrombolytic Therapy. Chest 2004; 126: 401S-428S.

43. Karabay O, Yetkin U, Onol H. Upper extremity deep vein thrombosis: clinical and treatment characteristics. J Int Med Res 2004; 32: 429-435. 
44. Oliver N, Short B, Thein M et al. Treatment of catheter-related deep vein thrombosis in patients with acute leukemia with anticoagulation. Leuk Lymphoma 2015; 14: 1-5 epub.

45. Frank DA, Meuse J, Hirsch D et al. The treatment and outcome of cancer patients with thromboses on central venous catheters. J Thromb Thrombolysis 2000; 10: 271-275.

46. Kovacs MJ, Kahn SR, Rodger M et al. A pilot study of central venous catheter survival in cancer patients using low molecular weight heparin (dalteparin) and warfarin without catheter removal for the treatment of upper extremity deep vein thrombosis (the catheter study). J Thromb Haemost 2007; 5 (8): 1650-3.

47. Kearon C, Akl EA, Comerota AJ et al. Antithrombotic therapy for VTE disease: Antithrombotic Therapy and Prevention of Thrombosis, 9th ed: American College of Chest Physicians Evidence-Based Clinical Practice Guidelines. Chest 2012; 141: e419S-494S.

48. Sabeti S, Schillinger M, Mlekusch W et al. Treatment of subclavian-axillary vein thrombosis: long-term outcome of anticoagulation versus systemic thrombolysis. Thromb Res 2002; 108: 279-285.

49. Ponec D, Irwin D, Haire WD et al. Recombinant tissue plasminogen activator (alteplase) for restoration of flow in occluded central venous access devices: a double-blind placebo-controlled trial--the Cardiovascular Thrombolytic to Open Occluded Lines (COOL) efficacy trial. J Vasc Interv Radiol 2001; 12: 951-955.

50. Monturo CA, Dickerson RN, Mullen JL. Efficacy of thrombolytic therapy for occlusion of long-term catheters. JPEN J Parenter Enteral Nutr 1990; 14: 312-314.

51. Mermel LA, Farr BM, Sherertz RJ et al. Guidelines for the management of intravascular catheter-related infections. Clin Infect Dis 2001; 32: 1249-1272.

52. O'Grady NP, Alexander M, Dellinger EP et al. Guidelines for the prevention of intravascular catheterrelated infections. Infect Control Hosp Epidemiol 2002; 23: 759-769.

53. Nouwen JL, Wielenga JJ, van Overhagen $\mathrm{H}$ et al. Hickman catheter-related infections in neutropenic patients: insertion in the operating theater versus insertion in the radiology suite. J Clin Oncol 1999; 17: 1304.

54. Raad, II, Hanna HA. Intravascular catheter-related infections: new horizons and recent advances. Arch Intern Med 2002; 162: 871-878.

55. Trautner BW, Darouiche RO. Catheter-associated infections: pathogenesis affects prevention. Arch Intern Med 2004; 164: 842-850.

56. Safdar N, Maki DG. Inflammation at the insertion site is not predictive of catheter-related bloodstream infection with short-term, noncuffed central venous catheters. Crit Care Med 2002; 30: 2632-2635.

57. Freeman R, King B. Recognition of infection associated with intravenous catheters. Br J Surg 1975; 62: 404-406.

58. Brun-Buisson C, Abrouk F, Legrand P et al. Diagnosis of central venous catheter-related sepsis. Critical level of quantitative tip cultures. Arch Intern Med 1987; 147: 873-877.

59. Cleri DJ, Corrado ML, Seligman SJ. Quantitative culture of intravenous catheters and other intravascular inserts. J Infect Dis 1980; 141: 781-786.

60. Sherertz RJ, Raad, II, Belani A et al. Three-year experience with sonicated vascular catheter cultures in a clinical microbiology laboratory. J Clin Microbiol 1990; 28: 76-82.

61. Maki DG, Weise CE, Sarafin HW. A semiquantitative culture method for identifying intravenous-catheterrelated infection. N Engl J Med 1977; 296: 1305-1309.

62. Catton JA, Dobbins BM, Kite $P$ et al. In situ diagnosis of intravascular catheter-related bloodstream infection: a comparison of quantitative culture, differential time to positivity, and endoluminal brushing. Crit Care Med 2005; 33: 787-791.

63. Safdar N, Fine JP, Maki DG. Meta-analysis: methods for diagnosing intravascular device-related bloodstream infection. Ann Intern Med 2005; 142: 451-466.

64. Abdelkefi A, Achour W, Ben Othman T et al. Difference in time to positivity is useful for the diagnosis of catheter-related bloodstream infection in hematopoietic stem cell transplant recipients. Bone Marrow Transplant 2005; 35: 397-401.

65. Sherertz RJ, Heard SO, Raad, II. Diagnosis of triple-lumen catheter infection: comparison of roll plate, sonication, and flushing methodologies. J Clin Microbiol 1997; 35: 641-646. 
66. Dobbins BM, Kite P, Catton JA et al. In situ endoluminal brushing: a safe technique for the diagnosis of catheter-related bloodstream infection. J Hosp Infect 2004; 58: 233-237.

67. Pronovost $\mathrm{P}$, Needham D, Berenholtz $\mathrm{S}$ et al. An intervention to decrease catheter-related bloodstream infections in the ICU. N Engl J Med 2006; 355: 2725-2732.

68. Pronovost P. Interventions to decrease catheter-related bloodstream infections in the ICU: the Keystone Intensive Care Unit Project. Am J Infect Control 2008; 36: S171 e171-175.

69. Eggimann $\mathrm{P}$, Hugonnet $\mathrm{S}$, Sax $\mathrm{H}$ et al. Long-term reduction of vascular access-associated bloodstream infection. Ann Intern Med 2005; 142: 875-876.

70. Moller T, Borregaard N, Tvede M, Adamsen L. Patient education--a strategy for prevention of infections caused by permanent central venous catheters in patients with haematological malignancies: a randomized clinical trial. J Hosp Infect 2005; 61: 330-341.

71. Henrickson KJ, Axtell RA, Hoover SM et al. Prevention of central venous catheter-related infections and thrombotic events in immunocompromised children by the use of vancomycin/ciprofloxacin/heparin flush solution: A randomized, multicenter, double-blind trial. J Clin Oncol 2000; 18: 1269-1278.

72. Recommendations for preventing the spread of vancomycin resistance. Recommendations of the Hospital Infection Control Practices Advisory Committee (HICPAC). MMWR Recomm Rep 1995; 44: 1-13.

73. Ljungman $P$, Hagglund $H$, Bjorkstrand $B$ et al. Peroperative teicoplanin for prevention of gram-positive infections in neutropenic patients with indwelling central venous catheters: a randomized, controlled study. Support Care Cancer 1997; 5: 485-488.

74. Lim SH, Smith MP, Machin SJ, Goldstone AH. A prospective randomized study of prophylactic teicoplanin to prevent early Hickman catheter-related sepsis in patients receiving intensive chemotherapy for haematological malignancies. Eur J Haematol Suppl 1993; 54: 10-13.

75. Veenstra DL, Saint S, Saha S et al. Efficacy of antiseptic-impregnated central venous catheters in preventing catheter-related bloodstream infection: a meta-analysis. Jama 1999; 281: 261-267.

76. Jaeger K, Zenz S, Juttner B et al. Reduction of catheter-related infections in neutropenic patients: a prospective controlled randomized trial using a chlorhexidine and silver sulfadiazine-impregnated central venous catheter. Ann Hematol 2005; 84: 258-262.

77. Logghe C, Van Ossel C, D'Hoore W et al. Evaluation of chlorhexidine and silver-sulfadiazine impregnated central venous catheters for the prevention of bloodstream infection in leukaemic patients: a randomized controlled trial. J Hosp Infect 1997; 37: 145-156.

78. Crnich CJ, Maki DG. Are antimicrobial-impregnated catheters effective? When does repetition reach the point of exhaustion? Clin Infect Dis 2005; 41: 681-685.

79. Crnich CJ, Maki DG. Are antimicrobial-impregnated catheters effective? Don't throw out the baby with the bathwater. Clin Infect Dis 2004; 38: 1287-1292.

80. McConnell SA, Gubbins PO, Anaissie EJ. Do antimicrobial-impregnated central venous catheters prevent catheter-related bloodstream infection? Clin Infect Dis 2003; 37: 65-72.

81. Falagas ME, Fragoulis K, Bliziotis IA, Chatzinikolaou I. Rifampicin-impregnated central venous catheters: a meta-analysis of randomized controlled trials. J Antimicrob Chemother 2007; 59: 359-369.

82. Hanna H, Benjamin R, Chatzinikolaou I et al. Long-term silicone central venous catheters impregnated with minocycline and rifampin decrease rates of catheter-related bloodstream infection in cancer patients: a prospective randomized clinical trial. J Clin Oncol 2004; 22: 3163-3171.

83. Hentrich $M$, Schalk $E$, Schmidt-Hieber $M$ et al. Central venous catheter-related infections in hematology and oncology: 2012 updated guidelines on diagnosis, management and prevention by the Infectious Diseases Working Party of the German Society of Hematology and Medical Oncology. Ann Oncol 2014; 25: 936-947.

84. Del Principe MI, Buccisano F, Maurillo $L$ et al. Infections increase the risk of central venous catheterrelated thrombosis in adult acute myeloid leukemia. Thromb Res 2013;132: 511-514.

85. Mehall JR, Saltzman DA, Jackson RJ, Smith SD. Fibrin sheath enhances central venous catheter infection. Crit Care Med 2002; 30: 908-912.

86. Herrmann M, Vaudaux PE, Pittet $D$ et al. Fibronectin, fibrinogen, and laminin act as mediators of adherence of clinical staphylococcal isolates to foreign material. J Infect Dis 1988; 158: 693-701. 
87. Choi G, Schultz MJ, Levi M, van der Poll T. The relationship between inflammation and the coagulation system. Swiss Med Wkly 2006; 136: 139-144.

88. Esmon CT. The interactions between inflammation and coagulation. Br J Haematol 2005; 131: 417-430.

89. Levi M, van der Poll T, Buller HR. Bidirectional relation between inflammation and coagulation. Circulation 2004; 109: 2698-2704.

90. Boersma RS, Schouten HC. Clinical practices concerning central venous catheters in haematological patients. Eur J Oncol Nurs 2010; 14: 200-204.

91. Shanks RM, Donegan NP, Graber ML et al. Heparin stimulates Staphylococcus aureus biofilm formation. Infect Immun 2005; 73: 4596-4606.

92. van Rooden CJ, Schippers EF, Guiot HF et al. Prevention of coagulase-negative staphylococcal central venous catheter-related infection using urokinase rinses: a randomized double-blind controlled trial in patients with hematologic malignancies. J Clin Oncol 2008; 26: 428-433.

93. Dillon PW, Jones GR, Bagnall-Reeb HA et al. Prophylactic urokinase in the management of long-term venous access devices in children: a Children's Oncology Group study. J Clin Oncol 2004; 22: 2718-2723.

94. Dumichen MJ, Seeger K, Lode HN et al. Randomized controlled trial of taurolidine citrate versus heparin as catheter lock solution in paediatric patients with haematological malignancies. J Hosp Infect 2012; 80: 304-309.

95. Slobbe L, Doorduijn JK, Lugtenburg PJ et al. Prevention of catheter-related bacteremia with a daily ethanol lock in patients with tunnelled catheters: a randomized, placebo-controlled trial. PLoS One 2010; 5: e10840.

96. Weijmer MC, Debets-Ossenkopp YJ, Van De Vondervoort FJ, ter Wee PM. Superior antimicrobial activity of trisodium citrate over heparin for catheter locking. Nephrol Dial Transplant 2002; 17: 2189-2195.

97. Weijmer MC, van den Dorpel MA, Van de Ven PJ et al. Randomized, clinical trial comparison of trisodium citrate 30\% and heparin as catheter-locking solution in hemodialysis patients. J Am Soc Nephrol 2005; 16: 2769-2777.

98. Boersma RS, Jie KS, Voogd AC et al. Concentrated citrate locking in order to reduce the long-term complications of central venous catheters: a randomized controlled trial in patients with hematological malignancies. Support Care Cancer 2015; 23: 37-45 epub ahead of print.

99. Ratcliffe $M$, Broadfoot $C$, Davidson $M$ et al. Thrombosis, markers of thrombotic risk, indwelling central venous catheters and antithrombotic prophylaxis using low-dose warfarin in subjects with malignant disease. Clin Lab Haematol 1999; 21: 353-357.

100.Fijnheer R, Paijmans B, Verdonck LF et al. Factor $V$ Leiden in central venous catheter-associated thrombosis. Br J Haematol 2002; 118: 267-270.

101. Harter C, Salwender HJ, Bach A et al. Catheter-related infection and thrombosis of the internal jugular vein in hematologic-oncologic patients undergoing chemotherapy: a prospective comparison of silvercoated and uncoated catheters. Cancer 2002; 94: 245-251.

102. Worth LJ, Seymour JF, Slavin MA. Infective and thrombotic complications of central venous catheters in patients with hematological malignancy: prospective evaluation of nontunneled devices. Support Care Cancer 2009; 17: 811-818.

103.Stoffel N, Rysler C, Buser A et al. Leukocyte count and risk of thrombosis in patients undergoing haematopoietic stem cell transplantation or intensive chemotherapy. Thromb Haemost 2010;103: 12281232.

104.Joks M, Czyz A, Poplawski D, Komarnicki M. Incidence and risk factors for central venous catheter-related thrombosis in hematological patients. Med Oncol 2014; 31: 772.

105. Morano SG, Coppola L, Latagliata R et al. Early and late complications related to central venous catheters in hematological malignancies: a retrospective analysis of 1102 patients. Mediterr J Hematol Infect Dis 2014; 6: e2014011.

106. Carratala J, Niubo J, Fernandez-Sevilla A et al. Randomized, double-blind trial of an antibiotic-lock technique for prevention of gram-positive central venous catheter-related infection in neutropenic patients with cancer. Antimicrob Agents Chemother 1999; 43: 2200-2204. 
CHAPTER 2

107.Karthaus M, Doellmann T, Klimasch T et al. Central venous catheter infections in patients with acute leukemia. Chemotherapy 2002; 48: 154-157.

108. Nosari A, Nichelatti M, De Gasperi A et al. Incidence of sepsis in central venous catheter-bearing patients with hematologic malignancies: preliminary results. J Vasc Access 2004; 5: 168-173.

109.Sakai T, Kohda K, Konuma $Y$ et al. A role for peripherally inserted central venous catheters in the prevention of catheter-related blood stream infections in patients with hematological malignancies. Int J Hematol 2014;100: 592-598. 


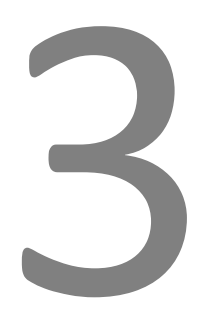

Clinical practices concerning central venous catheters in hematological patients

R.S. Boersma, H.C. Schouten

Eur J Oncol Nurs. 2010 Jul;14(3):200-204
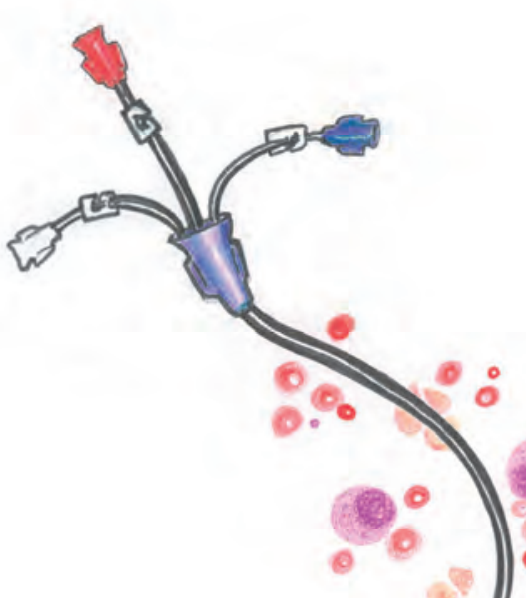


\section{ABSTRACT}

\section{Objective}

Central venous catheters (CVCs) are frequently used in patients with a hematological malignancy in order to administer chemotherapy, stem cell infusions, blood products, medication, parenteral hyperalimentation as well as for blood sampling.

Reported complications consist of mechanical complications during the insertion and long-term complications such as CVC-related thrombosis and infections. CVC-related thrombosis and infections are frequently occurring complications and may cause significant morbidity in patients with hematological malignancies. CVC-related infections and thrombosis should not be considered as a result of modern care or fait accompli and must be one of the priority targets of a multidisciplinary approach emphasizing quality-of-care improvement.

\section{Methods}

We conducted a survey among 23 Dutch and Belgian hematological centres to assess the local views and clinical practices concerning central venous catheters in hematological patients.

\section{Results and conclusions}

The local protocols and policies differ greatly among the centres probably reflecting wide differentiations in practice across Europe. It also shows lack of evidence concerning CVC-related thrombosis and infections which may cause morbidity in hematological patients. Further research has to be stimulated and development of clinical practice guidelines should be promoted. 


\section{INTRODUCTION}

Central venous catheters (CVCs) have considerably improved the management of patients with hematological malignancies, by facilitating chemotherapy, supportive therapy and blood sampling [1, 2]. Complications of insertion of CVCs include mechanical (arterial puncture, pneumothorax), thrombotic and infectious complications [3]. CVC-related thrombosis and infections are frequently occurring complications and may cause significant morbidity in patients with hematological malignancies. CVCrelated infections and thrombosis should not be considered as a result of modern care. Prevention of them should be one of the priority targets of a multidisciplinary approach. The optimum strategies to diagnose, prevent and treat CVC-related thrombosis and infections are not well known and this lack of knowledge is probably leading to variability in practice. We conducted a survey to assess the local views and clinical practices concerning central venous catheters in hematological patients. The goal of the survey was to evaluate the extent of variation in practice and to promote further research. With this, we hope to stimulate the future development of clinical practice guidelines in order to reduce the incidence of long-term complications like CVC-related infections and thrombosis in hematological patients.

\section{METHODS}

A survey amongst 17 Dutch and 6 Belgian hematological centres who are giving intensive chemotherapy according to the HOVON (Hemato Oncology Foundation for adults in the Netherlands) protocols was conducted in 2008. The survey contained 30 questions regarding local policies and protocols of catheter type, catheter insertion, catheter care, diagnosis, prevention and treatment of central venous catheter relatedthrombosis and infections in hematological patients. The survey was sent to an experienced hematologist in every centre.

\section{RESULTS}

All seventeen Dutch centres and five of the six Belgian centres responded (response 96\%). All replies were completed by at least one experienced staff member (consultant or senior nurse).

All centres treat adult patients with acute leukemias according the HOVON protocols and most of them perform autologous and allogeneic stem cell transplantations. The population of patients they treat is in need of a CVC for at least several weeks in order to administer chemotherapy, stem cell infusions, blood products, parenteral hyperalimentation and medication as well as for blood sampling. The patients 
experience long periods of severe neutropenia which makes them high risk for developing CVC-related infections.

The results of the questionnaire are summarized and discussed below.

\section{Insertion of the central venous catheter}

In nine (41\%) of the hematological centres the insertion of the CVC is performed by the hematologist. In six centres (27\%) the surgeon performs the insertion. The other insertions are done by the radiologist, anesthesiologist or critical care doctor (see pie 1).

Because most insertions are performed by the hematologist, most centres (16/22) insert untunneled CVCs in the subclavian vein on the nursing ward.

Only four centres insert a tunneled CVC in the jugular vein which is always done by the surgeon with the help of ultrasound.

The most widely used catheter for intensive chemotherapy and autologous stem cell transplantation is one with a double lumen. For unrelated allogeneic stem cell transplantation a CVC with three lumens is inserted in almost all centres. Only one centre inserts impregnated CVCs.

Most centres (15/22) have no preference for the left or right side.

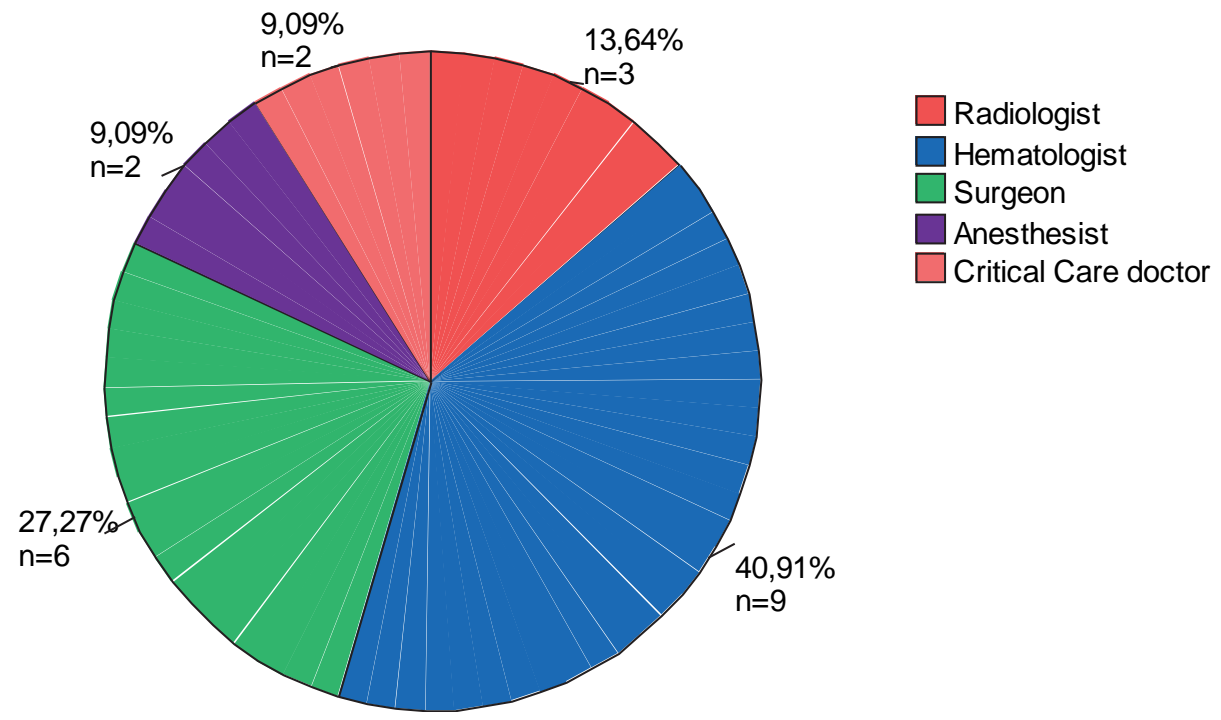

Pie 1. CVC Inserted by

Full barrier precautions during insertion of the CVC (sterile gloves, long-sleeved sterile gown, mask, cap and large sterile sheet drape) are taken in all centres.

Not one of the centres gives systemic antibiotic prophylaxis at the time of CVC insertion. 
Care of the central venous catheter

Chlorhexidine preparations are the most widely used antiseptics for cleansing the insertion site and hubs of the CVC in the Dutch and Belgian centres (12/22 centres). Three centres use alcohol, four centres use povidone iodine and two centres use a mixture of chlorhexidine and alcohol to clean the insertion site and catheter hub.

Semi-permeable transparent dressings are used by 20/22 centres. The other two centres use traditional gauze dressings.

In 8 of the 22 centres the patients wear a mask and cap during care of the CVC by the nurse. In only one centre does the patient takes care of his own CVC.

Catheter locking protocols differ greatly. The frequency of locking differs from once a day to once a week. The solutions used are citrate, urokinase, saline and heparin (see pie 2). The most widely used locking solution is heparin with concentrations ranging from $100 \mathrm{E} / \mathrm{ml}$ to $5000 \mathrm{E} / \mathrm{ml}$.
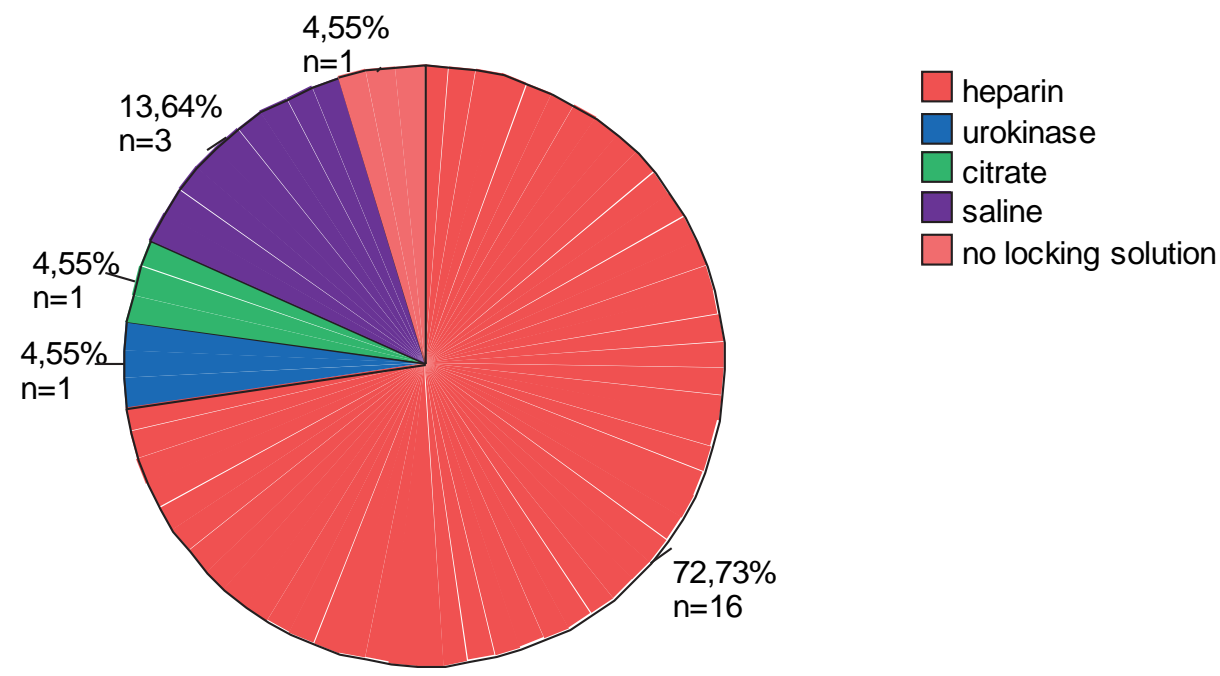

Pie 2. Locking solutions used

\section{CVC-related thrombosis}

All centres perform a compression ultrasound with Doppler as a first choice for the detection of CVC-related thrombosis. 
Standard prescription of anticoagulant prophylaxis was not performed by any of the centres. Only in one centre anticoagulant prophylaxis is given in case of known factor $\mathrm{V}$ Leiden carriership.

The treatment of CVC-related thrombosis consists of only low molecular weight heparins (LMWHs) in fourteen centres. Five centres give LMWHs followed by vitamin $\mathrm{K}$ antagonists (VKA). Only one centre removes the CVC and gives no additional anticoagulation. The duration of given anticoagulation differs greatly between 4 weeks to 6 months (see table 1 ).

In thirteen centres the CVC is removed in case of CVC-related thrombosis even if the CVC is still functional.

Table 1. Duration of treatment of CVC-related thrombosis

\begin{tabular}{llr}
\hline Duration of treatment & Number & $\%$ \\
\hline No anticoagulation & 1 & 4.5 \\
4 weeks & 2 & 9.0 \\
6 weeks & 1 & 4.5 \\
2 months & 1 & 4.5 \\
6 weeks to 3 months & 3 & 14.0 \\
3 months & 8 & 36.0 \\
3-6 months & 3 & 14.0 \\
Differs & 3 & 14.0 \\
Total & 22 & 100.0 \\
\hline
\end{tabular}

\section{CVC-related infections}

The diagnosis CVC-related infection is in most (12/22) centers made in case of persistent positive blood cultures. Six centres need a microbiologically positive tip to make a definitive diagnosis of a CVC-related infection. Only one of the centres uses new diagnostic methods, like differential time to positivity, in which the CVC can be left in situ.

One of the centres gives prophylactic antibiotic locks with vancomycin. Two centres give locks with gentamicin in case of positive line cultures. 


\section{DISCUSSION}

CVCs are an integral part of the care of hematological patients and their advantages are beyond doubt $[1,2]$. However long-term complications of a CVC like CVC-related infections and thrombosis cause morbidity and mortality in hematological patients [3]. This survey reveals that the local views and clinical practices concerning central venous catheters in hematological patients differ greatly between the Dutch and Belgian centres which probably reflects wide differentiations in practice across Europe.

\section{Insertion of the central venous catheter}

Whether or not tunneled CVCs are used is dependent in our centres of whom inserts the CVC. If the CVC is inserted by the hematologist untunneled CVCs in the subclavian vein are inserted. The benefit of tunneling long-term subclavian or jugular catheters is presently not known and requires further evaluation [4]. Well known is that experience of the person who inserts the CVC reduces the risk of thrombosis.

For surgeons use of ultrasound guidance is common. Not one of the hematologists uses ultrasound guidance. During venous catheterization, ultrasound guidance reduces the number of mechanical complications, the number of catheter placement failures, and the time required for insertion [5-8].

No matter who inserts the CVC, full barrier precautions during insertion of the CVC (sterile gloves, long-sleeved sterile gown, mask, cap and large sterile sheet drape) substantially reduces the incidence of CVC-related bloodstream infections compared with standard precautions (sterile gloves and small drape) (0.8/1000 and 0.5/1000 catheter-days, respectively; $P=0.02$ ) [9]. Full barrier precautions during insertion of the CVC should be, and as shown in our survey, are the standard of care during central venous catheter insertion.

Significant controversy keeps surrounding the usefulness of antiseptic-impregnated CVCs and CVCs impregnated with antimicrobial agents in hematological patients which can be seen in the practice of the Dutch and Belgian centres [10-12].

Systemic antibiotic prophylaxis at the time of CVC insertion is not recommended since no studies have demonstrated that oral or parenteral antibiotics might reduce the incidence of CVC-related bloodstream infections in hematological patients [13, 14].

\section{Care of the central venous catheter}

Since the microbes that colonize the catheter hub and the skin surrounding the insertion site are the source of most CVC-related infections, prevention of CVC-related 
infections focuses mainly on the essential measures of aseptic insertion technique and proper catheter care.

Continuing quality improvement programs to assure compliance with catheter care guidelines significantly reduce CVC-related bloodstream infections [14, 15].

Moller et al showed a reduction of $>50 \%$ in the incidence rate of CVC-related infections with a systematic, individualized, supervised patient education program and self-care for the catheter [16]. In our survey in only once centre takes the patient care of his own CVC.

An effective skin-preparation agent cleans away microbes, then continues to inhibit pathogen growth between dressing changes. This was traditionally accomplished with iodophor or alcohol. Chlorhexidine demonstrated in studies significant residual antimicrobial effect over 5 days and was more effective than isopropyl alcohol, alcohol or povidone iodine alone. Based on these studies best-practice guidelines from the CDC, Agency on Healthcare Research and Quality, Infusion Nurses Society (INS), and Institute for Healthcare improvement now prefer a $2 \%$ chlorhexidine solution $[14,17,18]$.

As is shown in our survey this advice is followed by most of the Dutch and Belgian centres.

Transparent, semipermeable dressings are a popular means of dressing catheter insertion sites as is shown in the results of the survey. In a meta-analysis that compared transparent dressings versus groups using gauze dressings no difference in CVC-related bloodstream infections was found. The choice of dressing can be a matter of preference $[14,19]$.

More important than the choice of the dressing is to replace gauze dressings every 48 hours and transparent dressings at least every 7 days and to change the dressing when it becomes damp, loose, has visible blood or is soiled.

Considerable uncertainty persists about the optimum choice of 'locking' protocol leading to variability in practice as is shown in the results of the survey. Locking of the catheter lumens with an anticoagulant agent is performed to avoid catheter clotting and CVC-related thrombosis and infections. The most widely used locking solution in the Dutch and Belgian centres is heparin. This is probably as a result of studies published between 1979 and 1996 that showed heparin infusion effectively reduces CVC-related thrombus formation and may reduce catheter infection [20].

Since heparin has side-effects like unintentional systemic anticoagulation, interference with lab assessments of clotting, heparin-induced thrombocytopenia and enhances staphylococcal biofilm formation alternatives to heparin are studied [21].

Much has been published about urokinase and trisodium citrate as alternatives to heparin. Studies have shown that not only thrombotic occlusion but also CVC-related infections may be due to fibrin deposition associated with catheters [22]. Locking with 
urokinase decreases fibrin deposition and thrombus formation and in this way decreases the risk of CVC-related bloodstream infections and thrombosis [23-25].

Trisodium citrate has been shown in prospective randomized controlled trials to be an effective antimicrobial and anticoagulant catheter locking agent in hemodialysis patients [24]. This effect warrants further investigation in hematological patients.

\section{CVC-related thrombosis}

The strategies of diagnosing and preventing CVC-related thrombosis are mostly the same in every centre in our survey.

As is shown in our survey the treatment of CVC-related thrombosis in hematological patients remains a matter of debate with no consensus which can only be clarified in future by further research. Specific attention to the preservation of the CVC should be made in these hematological patients.

\section{CVC-related infections}

As is shown in the variability of practice between the centres concerning the diagnosis of a CVC-related infection, diagnosing CVC-related infections is notoriously difficult, especially when the CVC is left in situ. Most centres use persistent positive blood cultures or microbiologically positive tips to make a definitive diagnosis of a CVC-related infection.

Only one of the Dutch and Belgian centers uses new diagnostic methods like differential time to positivity which has the advantage that the CVC can be left in situ. In future much effort must be made to promote new diagnostic methods allowing the CVC to be left in situ.

Since prevention of CVC-related infections is of utmost importance one of the centres gives antibiotic lock prophylaxis with vancomycin. This type of prevention has shown to significantly increase the time to develop a CVC-related infection and to decrease the amount of gram positive CVC-related infections [26]. However, the Centers for Disease Control and Prevention guidelines recommend against prophylactic use of vancomycin because this is an independent risk factor for acquisition of vancomycin-resistant enterococci [27].

\section{CONCLUSION}

The local protocols and policies differ greatly among the Dutch and Belgian hematological centres probably reflecting wide differentiations in practice across Europe. It also reflects important lack of evidence concerning CVC-related thrombosis 


\section{CHAPTER 3}

and infections which may cause morbidity in hematological patients. CVC-related thrombosis and infections should not be considered as a result of modern care and prevention of them is of utmost importance. The target should be a multidisciplinary approach to decrease the risk of CVC-related thrombosis and infections to zero. This must be done by strict adherence to known antiseptic techniques and stimulation of further research in order to develop best clinical practice guidelines. 


\section{REFERENCES}

1. Kumagai T, Sakamaki H, Tanikawa S et al. Utility and safety of Hickman catheters for venous access after bone marrow transplantation. Intern Med 1998; 37: 286-291.

2. Hickman RO, Buckner CD, Clift RA et al. A modified right atrial catheter for access to the venous system in marrow transplant recipients. Surg Gynecol Obstet 1979; 148: 871-875.

3. McGee DC, Gould MK. Preventing complications of central venous catheterization. N Engl J Med 2003; 348: 1123-1133.

4. Andrivet $P$, Bacquer $A$, Ngoc CV et al. Lack of clinical benefit from subcutaneous tunnel insertion of central venous catheters in immunocompromised patients. Clin Infect Dis 1994; 18: 199-206.

5. Hind D, Calvert N, McWilliams R et al. Ultrasonic locating devices for central venous cannulation: metaanalysis. BMJ 2003; 327: 361.

6. Biffi R, Orsi F, Pozzi S et al. Best choice of central venous insertion site for the prevention of catheterrelated complications in adult patients who need cancer therapy: a randomized trial. Ann Oncol 2009; 20: 935-940.

7. Teichgraber UK, Benter T, Gebel M, Manns MP. A sonographically guided technique for central venous access. AJR Am J Roentgenol 1997; 169: 731-733.

8. Randolph AG, Cook DJ, Gonzales CA, Pribble CG. Ultrasound guidance for placement of central venous catheters: a meta-analysis of the literature. Crit Care Med 1996; 24: 2053-2058.

9. Raad, II, Hohn DC, Gilbreath BJ et al. Prevention of central venous catheter-related infections by using maximal sterile barrier precautions during insertion. Infect Control Hosp Epidemiol 1994; 15: 231-238.

10. Logghe C, Van Ossel C, D'Hoore W et al. Evaluation of chlorhexidine and silver-sulfadiazine impregnated central venous catheters for the prevention of bloodstream infection in leukaemic patients: a randomized controlled trial. J Hosp Infect 1997; 37: 145-156.

11. Veenstra DL, Saint S, Saha S et al. Efficacy of antiseptic-impregnated central venous catheters in preventing catheter-related bloodstream infection: a meta-analysis. Jama 1999; 281: 261-267.

12. McConnell SA, Gubbins PO, Anaissie EJ. Do antimicrobial-impregnated central venous catheters prevent catheter-related bloodstream infection? Clin Infect Dis 2003; 37: 65-72.

13. Ljungman $P$, Hagglund $H$, Bjorkstrand $B$ et al. Peroperative teicoplanin for prevention of gram-positive infections in neutropenic patients with indwelling central venous catheters: a randomized, controlled study. Support Care Cancer 1997; 5: 485-488.

14. O'Grady NP, Alexander M, Dellinger EP et al. Guidelines for the prevention of intravascular catheterrelated infections. Infect Control Hosp Epidemiol 2002; 23: 759-769.

15. Eggimann $\mathrm{P}$, Hugonnet $\mathrm{S}, \mathrm{Sax} \mathrm{H}$ et al. Long-term reduction of vascular access-associated bloodstream infection. Ann Intern Med 2005; 142: 875-876.

16. Moller T, Borregaard N, Tvede M, Adamsen L. Patient education--a strategy for prevention of infections caused by permanent central venous catheters in patients with haematological malignancies: a randomized clinical trial. J Hosp Infect 2005; 61: 330-341.

17. Moureau NL. Are your skin-prep and catheter maintenance techniques up-to-date? Nursing 2009; 39: 1516.

18. Hibbard JS, Mulberry GK, Brady AR. A clinical study comparing the skin antisepsis and safety of ChloraPrep, 70\% isopropyl alcohol, and 2\% aqueous chlorhexidine. J Infus Nurs 2002; 25: 244-249.

19. Hoffmann KK, Weber DJ, Samsa GP, Rutala WA. Transparent polyurethane film as an intravenous catheter dressing. A meta-analysis of the infection risks. JAMA 1992; 267: 2072-2076.

20. Randolph AG, Cook DJ, Gonzales CA, Andrew M. Benefit of heparin in central venous and pulmonary artery catheters: a meta-analysis of randomized controlled trials. Chest 1998; 113: 165-171.

21. Shanks RM, Donegan NP, Graber ML et al. Heparin stimulates Staphylococcus aureus biofilm formation. Infect Immun 2005; 73: 4596-4606.

22. Mehall JR, Saltzman DA, Jackson RJ, Smith SD. Fibrin sheath enhances central venous catheter infection. Crit Care Med 2002; 30: 908-912. 
CHAPTER 3

23. Klerk CP, Smorenburg SM, Buller HR. Thrombosis prophylaxis in patient populations with a central venous catheter: a systematic review. Arch Intern Med 2003; 163: 1913-1921.

24. Winnett G, Nolan J, Miller M, Ashman N. Trisodium citrate $46.7 \%$ selectively and safely reduces staphylococcal catheter-related bacteraemia. Nephrol Dial Transplant 2008; 23: 3592-3598.

25. van Rooden CJ, Schippers EF, Guiot HF et al. Prevention of coagulase-negative staphylococcal central venous catheter-related infection using urokinase rinses: a randomized double-blind controlled trial in patients with hematologic malignancies. J Clin Oncol 2008; 26: 428-433.

26. Henrickson KJ, Axtell RA, Hoover SM et al. Prevention of central venous catheter-related infections and thrombotic events in immunocompromised children by the use of vancomycin/ciprofloxacin/heparin flush solution: A randomized, multicenter, double-blind trial. J Clin Oncol 2000; 18: 1269-1278.

27. Recommendations for preventing the spread of vancomycin resistance. Recommendations of the Hospital Infection Control Practices Advisory Committee (HICPAC). MMWR Recomm Rep 1995; 44: 1-13. 


\section{4}

Concentrated citrate locking in order to reduce the long term complications of central venous catheters: a randomized controlled trial in patients with hematological malignancies
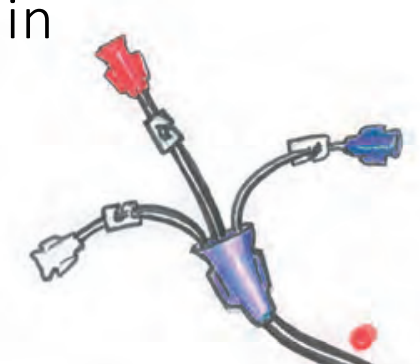

$x$

R.S. Boersma, K.S. Jie, A.C. Voogd, K. Hamulyak, A. Verbon and H.C. Schouten 


\section{ABSTRACT}

\section{Objective}

Central venous catheter (CVC)-related thrombosis and infections are frequently occurring complications and may cause significant morbidity in patients with hematological malignancies. Interventions to decrease fibrin deposition have the potential to reduce CVC-related thrombosis and infections. At present heparin is most often used as a locking solution for central venous catheters in hematological patients despite a lack of evidence regarding the efficacy and safety. Trisodium citrate (TSC) had been shown to be an effective antimicrobial catheter locking in hemodialysis patients. We performed a prospective randomized phase III multicenter trial to determine the efficacy of TSC as a locking solution compared to heparin in preventing CVC-related thrombosis and infections in patients with hematological malignancies.

\section{Patients and methods}

The study was conducted between July 2006 and August 2010 at the University Hospital Maastricht and the Atrium Medical Center Parkstad Heerlen, The Netherlands. Consecutive adult patients with hematological malignancies who were going to receive a CVC for intensive chemotherapy were included. The primary endpoints were (a)symptomatic CVC-related thrombosis and CVC-related infections.

\section{Results}

Thirty-four episodes of CVC-related bloodstream infections (CVC-BSI) occurred in the 108 patients who were randomized to locking with heparin compared with 35 episodes in the 99 patients who were randomized to locking with TSC ( $p=0.654)$. We did find seven times more CVC-BSI with Gram negative rods in CVCs locked with heparin ( $p=$ 0.041).

The cumulative incidence of symptomatic thrombosis was $10 \%$ in the heparin group and $5 \%$ in the TSC group (hazard ratio 0.525; 95\% confidence interval 0.182-1.512).

\section{Conclusion}

Locking with TSC in patients with hematological malignancies undergoing intensive chemotherapy significantly reduced the incidence of CVC-BSI with gram negative rods. However, the incidence of CVC-BSI with coagulase-negative staphylococcus or CVCrelated thrombosis was not reduced by TSC locking. 


\section{INTRODUCTION}

Central venous catheters (CVCs) have considerably improved the management of patients with hematological malignancies by facilitating chemotherapy, supportive therapy and blood sampling. Complications of CVCs include problems during insertion (arterial puncture, pneumothorax) and long-term complications like thrombotic and infectious complications [1]. CVC-related thrombosis and infections are frequently occurring complications and may cause significant morbidity in patients with hematological malignancies. CVC-related thrombosis and infections have a bidirectional relationship [2-4]. The incidence of symptomatic CVC-related thrombosis and CVCrelated bloodstream infections (CVC-BSI) has been reported to vary between 1.2-18.0\% and $0.0-40.6 \%$ respectively in patients with hematological malignancies [5-7].

Fibrin sheath formation around the external portion of the CVC and within the catheter lumen has been implicated as a major contributing factor in both occlusive and infectious events [8]. Interventions to decrease fibrin deposition and thrombus formation have the potential to reduce CVC-related thrombosis and infections $[9,10]$.

At present, due to its anticoagulant properties, most often heparin is used as locking solution for CVCs in hematological patients despite a lack of evidence regarding the efficacy and safety [7, 11, 12]. Furthermore heparin is associated with potential systemic anticoagulation, heparin induced thrombocytopenia and bleeding risks [13].

Trisodium citrate (TSC) prevents CVC-related thrombosis via local anticoagulation by chelating ionized calcium. TSC exerts a broad antimicrobial and anti-yeast effect [14]. TSC has been shown to be an effective antimicrobial catheter locking solution in hemodialysis patients. TSC lowered the incidence of CVC-related bloodstream infections in hemodialysis patients from 4.1 CVC-related BSI per 1000 catheter days to 1.1 per 1000 catheter days. TSC is at least as effective as heparin in maintaining catheter patency in hemodialysis patients [15-17].

Therefore, we performed a randomized controlled clinical trial to determine the efficacy of TSC as a locking solution compared to heparin in preventing CVC-related infections and thrombosis in patients with hematological malignancies undergoing intensive chemotherapy.

\section{METHODS}

\section{Design of the study}

This prospective, multicenter, randomized controlled study was conducted between July 2006 and August 2010 at the University Hospital Maastricht (a tertiary referral center for hematological diseases in the Netherlands) and the Atrium Medical Center Parkstad Heerlen (a tertiary medical teaching hospital), The Netherlands. 
The study protocol was approved by the medical ethical committees. All procedures followed were in accordance with the ethical standards of the responsible committee on human experimentation and with the ethical standards laid down in the 1964 Declaration of Helsinki. All participating patients gave written informed consent.

\section{Endpoints}

The main endpoints of the study were (a)symptomatic thrombosis and/or CVC-related infections.

Secondary endpoints were premature removal of the catheter, failure to aspirate blood, hypocalcemic symptoms or severe bleeding. Severe bleeding was present if one the following occurred: CNS bleeding, bleeding with hypotension (systolic blood pressure $<80 \mathrm{~mm} \mathrm{Hg}$ or a $30-\mathrm{mm} \mathrm{Hg}$ decrease in systolic blood pressure) or a $>2$ $\mathrm{mmol} / \mathrm{I}$ decrease in hemoglobin in any 24-hour period.

\section{Patients}

Consecutive adult patients with hematological malignancies who were going to receive a tunneled or non-tunneled CVC for intensive chemotherapy, including patients for stem cell transplantation, were eligible for the study. All patients were treated according to a local protocol with ciprofloxacin and fluconazol to prevent infections with Gram negative rods and candida spp.

Exclusion criteria were the presence of a CVC at admission, history of CVC-related thrombosis and/or infection, indication for anticoagulant treatment or prophylaxis, patients with totally implanted catheters and CVCs impregnated with antimicrobial agents. Patients were centrally randomized by using sealed envelopes to have each lumen of their CVC locked after use with either heparin sodium $5000 \mathrm{IE} / \mathrm{ml}$ or trisodium citrate $46.7 \%$ (Citralock, Dirinco). To obtain a continuing balance of treatments, randomization was divided into consecutive blocks of 20 patients.

Each patient could only be included and randomized once.

\section{Catheter insertion and management}

CVCs were tunneled polyurethane double-lumen catheters. The CVCs were inserted by an experienced surgeon in the operating room. CVCs were inserted in the right jugular or subclavian vein, if possible. Catheters were inserted percutaneously by using the Seldinger technique and most CVCs were placed with the help of ultrasound. The CVC tip was confirmed radiographically to lie in the distal superior vena cava/right atrium. No antibiotic prophylaxis specifically for CVC-related infections was given.

Depending on clinical needs, the CVC was either locked after each treatment cycle or used continuously for a limited number of days until switching to treatment cycles 
with intermittent locking. The CVCs were used for administering cytotoxic drugs and supportive treatment (i.e.fluids, blood products, parenteral feeding, and antimicrobial therapy). Nurses were allowed to draw blood from the CVC for diagnostic purposes.

After each use, the catheter lumen was flushed with $10 \mathrm{ml} 0.9 \% \mathrm{NaCl}$ and then locked with either heparin $5000 \mathrm{IU} / \mathrm{ml}$ or $46.7 \%$ TSC using a volume exactly equivalent to the internal volume noted on each catheter.

\section{CVC-related infections}

In case of fever (temperature above 38.5 degrees celcius) at least two sets of blood drawn for culture, with at least one set drawn peripherally, were taken at the same time. Empirical iv antimicrobial therapy (piperacillin/tazobactam or ceftazidim) was started thereafter. Empirical therapy was discontinued if blood cultures remained negative for 72 hours. If a bacteremia was diagnosed, empirical therapy was adjusted to the most appropriate small-spectrum regimen.

CVCs were removed aseptically, at the discretion of attending physicians or if they were no longer needed. If a catheter was removed because of suspicion of an infection a $5-\mathrm{cm}$ segment of the removed catheter tip was aseptically cut and delivered to the microbiology laboratory for quantitative cultures using the roll plate method.

The definitions of central venous catheter-related infections according to the Infectious Diseases Society of America (IDSA) and The Healthcare Infection Control Practices Advisory Committee (HICPAC) were used in this study [18]. A probable CVC-BSI is a bacteremia in a patient who has an intravascular device and $>1$ positive result of culture of blood samples obtained from the peripheral vein, clinical manifestations of infection, and no apparent source for bloodstream infection (with the exception of the catheter).

A definite CVC-BSI is a bacteremia in a patient who has an intravascular device and $>1$ positive result of culture of blood samples obtained from the peripheral vein, clinical manifestations of infection, and no apparent source for bloodstream infection and either a catheter tip with the same micro-organism or a differential time to positivity (a positive result of a culture from a CVC that is obtained at least 2 hours earlier than a positive result of culture from peripheral blood). Diagnosis and management of catheter-related infections was performed according to the published recommendations of the IDSA [18].

In case of recurrent bacteremia, an interval between positive cultures of blood samples of more than seven days was counted as 2 episodes of CVC-related infections. 


\section{CVC-related thrombosis}

Patients with a clinical suspicion of a (symptomatic) CVC-related thrombosis were referred to the department of Radiology for Doppler ultrasound. CVC-related thrombosis was diagnosed in case of non-compressibility of the vein, visualization of an echogenic intraluminal mass, and absence of respiratory variation, or monophasic flow [19]. In our study, a systematically examination by ultrasonography was, if possible, planned 30 days after insertion of the CVC and within 24 hours after removal of the CVC. The ultrasound examination was performed bilaterally.

\section{STATISTICAL ANALYSIS}

The study was powered to find a reduction in (a)symptomatic CVC-related thrombosis and/or infections of minimally 50\%, with an anticipated incidence rate of $30 \%$ in those receiving heparin. With 120 patients in the standard arm (heparin) and 120 patients in the TSC arm, there will be a 76\% chance of detecting a significant difference at a twosided $0.05 \%$ significance level, assuming that the occurrence of each primary endpoint is reduced from $30 \%$ to $15 \%$. In order to overcome $5-10 \%$ possible dropouts due to ineligibility and non-evaluability a total of 260 patients were planned for this study.

Baseline characteristics for continuous variables were expressed, depending on the distribution of the data, as mean (range) or median (range). Differences in baseline characteristics between the two groups were analyzed using the chi-squared test, independent-samples T test or in case of non-normal distribution the Mann-Whitney $U$ test.

The primary outcomes were dichotomously scored. Differences between the heparin and TSC group were analyzed using the Chi-squared test, Cox regression analyses, and a Kaplan- Meier analysis. Statistical difference was established at $P<0.05$. All analysis were performed on an intention to treat basis. All analyses were performed using SPSS version 20.0.

\section{RESULTS}

Between July 2006 and August 2010 two hundred and twelve patients were randomly assigned at both institutes with a planned accrual of 260 patients. Ultimately 207 patients were analyzed (see figure 1). One hundred and eight patients were assigned to group A (heparin) and 99 patients were assigned to group B (TSC). Baseline patient and CVC characteristics were not statistically different between the study arms (table 1). 


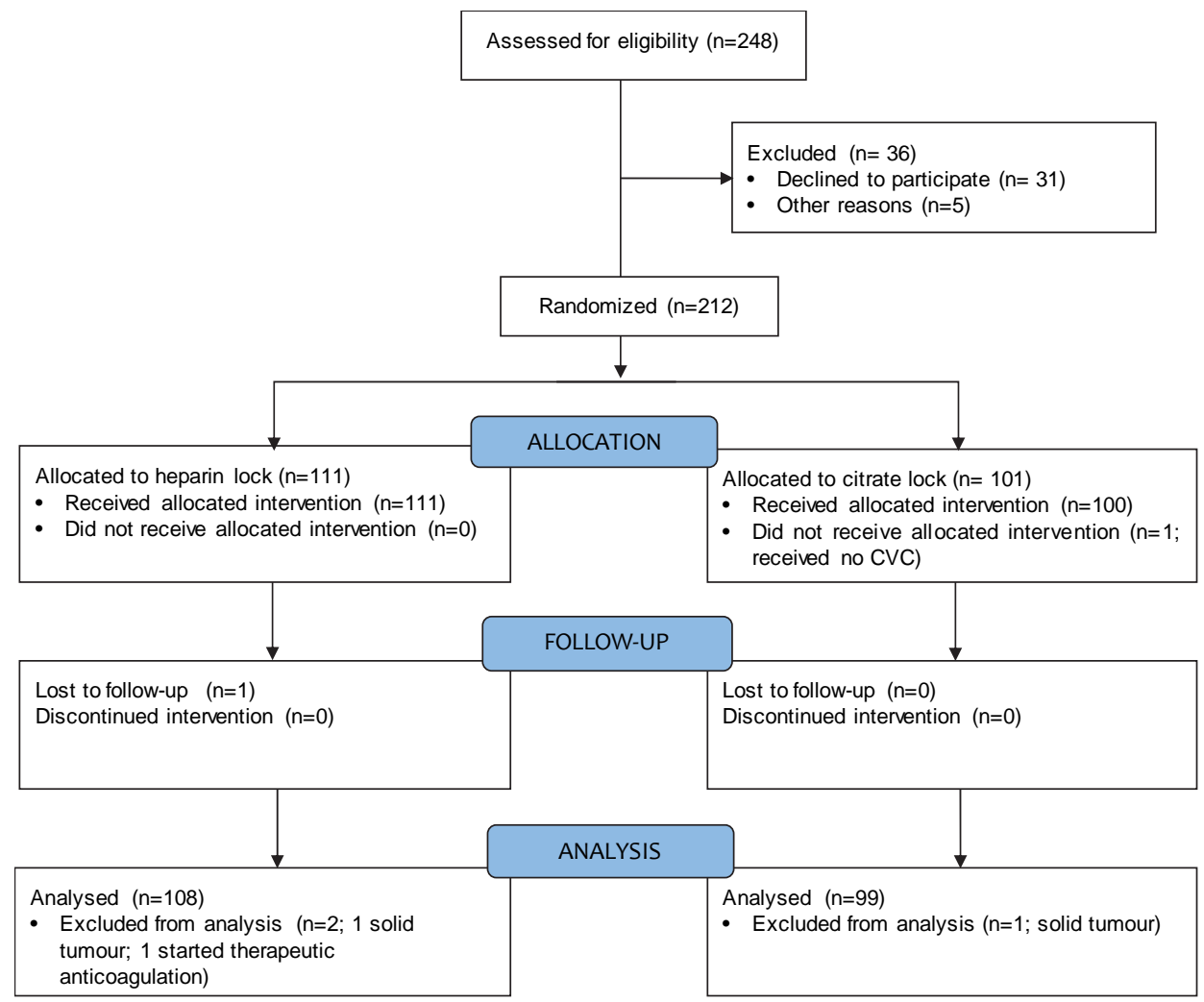

Fig. 1 CONSORT 2010 flow diagram

\section{CVC dwell time}

The mean duration of the CVC being in place was 86 days (standard deviation 91.3 days) in the heparin group and 75 days (standard deviation 63.9 days) in the TSC group ( $P=$ $0.475)$. There were 9288 catheter days in the heparin group and 7425 catheter days in the TSC group (table 1). 
Table 1. Baseline characteristics of the population analyzed

\begin{tabular}{|c|c|c|c|c|c|}
\hline & \multicolumn{2}{|c|}{ Heparin group $(n=108)$} & \multicolumn{2}{|c|}{ Citrate group ( $n=99)$} & \multirow[t]{2}{*}{$P$-value } \\
\hline & $\mathrm{N}$ & $(\%)$ & $\mathrm{N}$ & $(\%)$ & \\
\hline mean age, years & \multicolumn{2}{|l|}{55.2} & \multicolumn{2}{|l|}{54.1} & \multirow[t]{3}{*}{ NS } \\
\hline median age, years & \multicolumn{2}{|l|}{58.4} & \multicolumn{2}{|l|}{56.6} & \\
\hline standard deviation & \multicolumn{2}{|l|}{11.2} & \multicolumn{2}{|l|}{13.1} & \\
\hline Male/female & \multicolumn{2}{|l|}{$60 / 48$} & \multicolumn{2}{|l|}{$68 / 31$} & NS \\
\hline \multicolumn{6}{|l|}{ Centre } \\
\hline MUMC & 98 & 90 & 94 & 95 & \multirow[t]{2}{*}{ NS } \\
\hline Atrium Heerlen & 10 & 10 & 5 & 5 & \\
\hline \multicolumn{6}{|l|}{ Insertion place } \\
\hline Internal jugular vein & 101 & 94 & 96 & 97 & \multirow[t]{2}{*}{ NS } \\
\hline Subclavian vein & 7 & 6 & 3 & 3 & \\
\hline \multicolumn{6}{|l|}{ CVC dwell time } \\
\hline mean, days & \multicolumn{2}{|l|}{86} & \multicolumn{2}{|l|}{75} & \multirow[t]{3}{*}{$0.475^{*}$} \\
\hline median, days & \multicolumn{2}{|l|}{63} & \multicolumn{2}{|l|}{54} & \\
\hline standard deviation & \multicolumn{2}{|l|}{91.3} & \multicolumn{2}{|l|}{63.9} & \\
\hline Catheter days & \multicolumn{2}{|l|}{9288} & 7425 & & \\
\hline Disease & & & & & \\
\hline AML & 35 & & 31 & & NS \\
\hline ALL & 11 & & 7 & & \\
\hline AUL & 2 & & 0 & & \\
\hline Myeloma & 21 & & 22 & & \\
\hline Lymphoma & 30 & & 26 & & \\
\hline Aplastic anemia & 1 & & 2 & & \\
\hline CML & 2 & & 0 & & \\
\hline Myelodysplasia & 3 & & 7 & & \\
\hline Amyloidosis & 1 & & 3 & & \\
\hline Myelofibrosis & 1 & & 1 & & \\
\hline CLL & 1 & & 0 & & \\
\hline Treatment & & & & & \\
\hline Autologous BMT & 41 & & 40 & & NS \\
\hline Allogeneic BMT & 18 & & 15 & & \\
\hline Intensive CTX & 40 & & 39 & & \\
\hline ATG & 1 & & 1 & & \\
\hline Chemotherapy & 7 & & 4 & & \\
\hline None & 1 & & 0 & & \\
\hline Duration of severe neu & & & & & \\
\hline mean, days & 24.8 & & 22.9 & & NS \\
\hline median, days & 12.0 & & 11.0 & & \\
\hline standard deviation & 27.7 & & 23.6 & & \\
\hline Neutropenia at insertio & & & & & \\
\hline yes & 14 & 13 & 15 & 15 & NS \\
\hline
\end{tabular}

*Mann-Whitney $-U$ Test used

** Neutrophil count $<500$ cells/ul

MUMC, University hospital Maastricht 
$A M L$, acute myeloid leukemia; $A L L$, acute lymphoblastic leukemia; $A U L$, acute undifferentiated leukemia; $C M L$, chronic myeloid leukemia; CLL, chronic lymphocytic leukemia; BMT, bone marrow transplantation; CTX, chemotherapy; ATG, anti-thymocyte globulin; VCS, superior vena cava; NS, not significant

\section{CVC-RELATED THROMBOSIS}

The cumulative incidence of symptomatic thrombosis was $10 \%$ in the heparin group and $5 \%$ in the TSC group ( $P=0.167$; table 2$)$. The calculated hazard ratio was 0.525 (95\% confidence interval, 0.182-1.512). Only one patient with a symptomatic CVC-related thrombosis in the heparin group developed a symptomatic pulmonary embolism and none in the TSC group. Five patients with a symptomatic CVC-related thrombosis had been diagnosed with an acute myeloid leukemia, four patients with an acute lymphoblastic leukemia, two with a multiple myeloma and five patients with a lymphoma. The mean number of days between insertion of the CVC and CVC-related thrombosis was 30 days (standard deviation 19.7 days) in the heparin group and 67 days (standard deviation 38.5 day) in the TSC group ( $P=0.224$; figure 2 ).

The cumulative incidence of asymptomatic thrombosis was $21 / 79(27 \%)$ in the heparin group and 22/87 (25\%) in the TSC group ( $P=0.849$; table 2$)$.

Table 2. Thrombotic events

\begin{tabular}{|c|c|c|c|c|c|}
\hline \multirow[t]{2}{*}{ No. of patients } & \multicolumn{2}{|c|}{ Heparin group $(n=108)$} & \multicolumn{2}{|c|}{ Citrate group ( $n=99)$} & \multirow[t]{2}{*}{ P-value } \\
\hline & $\mathrm{N}$ & $(\%)$ & $\mathrm{N}$ & $(\%)$ & \\
\hline Asymptomatic VTE & \multicolumn{2}{|c|}{ total 79 pts } & \multicolumn{2}{|c|}{ total $87 \mathrm{pts}$} & 0.849 \\
\hline yes & 21 & 27 & 22 & 25 & \\
\hline no & 58 & 73 & 65 & 75 & \\
\hline Symptomatic VTE & \multicolumn{2}{|c|}{ total $108 \mathrm{pts}$} & \multicolumn{2}{|c|}{ total 99 pts } & 0.167 \\
\hline yes & 11 & 10 & 5 & 5 & \\
\hline no & 97 & 90 & 94 & 95 & \\
\hline Time to VTE & & & & & 0.224 \\
\hline mean, days & \multicolumn{2}{|l|}{29.7} & \multicolumn{2}{|l|}{67.0} & \\
\hline median, days & \multicolumn{2}{|l|}{26.0} & \multicolumn{2}{|l|}{56.0} & \\
\hline standard deviation & \multicolumn{2}{|l|}{19.7} & \multicolumn{2}{|l|}{38.5} & \\
\hline
\end{tabular}




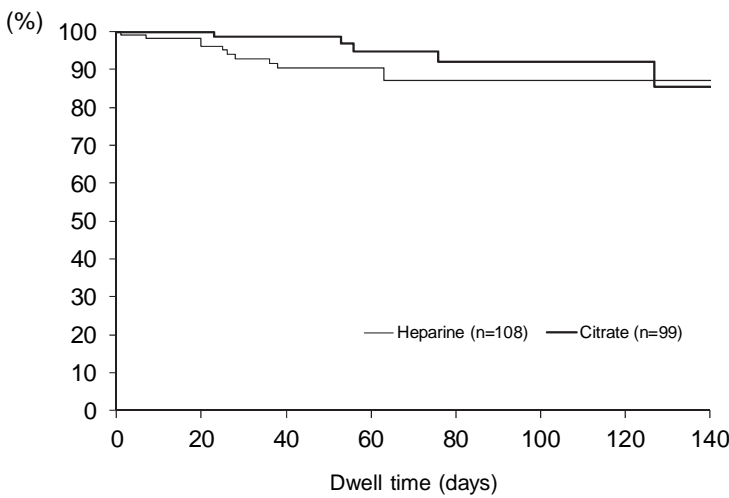

Fig. 2 Kaplan-Meier analysis of time to CVC-related thrombosis in TSC and heparin group $P=0.224$

\section{CVC-related infections}

There were 77 CVC-related infections in the heparin group and 75 CVC-related infections in the TSC group which is shown in table 3. Thirty-four episodes of probable or definite CVC-BSI occurred in the 108 patients with heparin locking (31\%) and 35 episodes in the 99 patients with TSC locking (35\%) (NS; table 4). The calculated hazard ratio for the first CVC-BSI was 1.156 (95\% confidence interval, 0.671-1,992). In 46\% of CVC-BSI cultures yielded gram-positive micro-organisms, predominantly coagulasenegative staphylococci (CNS) and Staphylococcus hemolyticus (table 4).

Table 3. Infectious events

\begin{tabular}{|c|c|c|c|c|c|}
\hline & \multicolumn{2}{|c|}{ Heparin group $(n=108)$} & \multicolumn{2}{|c|}{ Citrate group ( $n=99)$} & \multirow[t]{2}{*}{ P-value - } \\
\hline & $\mathrm{N}$ & $(\%)$ & $\mathrm{N}$ & $(\%)$ & \\
\hline \multicolumn{6}{|l|}{ CVC-related infections, total } \\
\hline Yes & 77 & & 75 & & \\
\hline CVC BSI total ${ }^{*}$ & & & & & 0.654 \\
\hline Yes & 34 & 31 & 35 & 35 & \\
\hline No & 74 & 69 & 64 & 65 & \\
\hline CVC BSI/1000 days & 3.7 & & 4.7 & & \\
\hline \multicolumn{6}{|l|}{ CVC-related infections } \\
\hline exit site infection & 3 & & 2 & & \\
\hline tunnel infection & 4 & & 3 & & \\
\hline catheter colonization & 1 & & 1 & & \\
\hline catheter contamination & 0 & & 4 & & \\
\hline positive CVC blood culture & 35 & & 30 & & \\
\hline probable CVC BSI & 18 & & 22 & & \\
\hline definite CVC BSI & 16 & & 13 & & \\
\hline non CVC BSI & 5 & & 5 & & \\
\hline
\end{tabular}




\begin{tabular}{|c|c|c|c|c|c|}
\hline & \multicolumn{2}{|c|}{ Heparin group $(n=108)$} & \multicolumn{2}{|c|}{ Citrate group $(n=99)$} & \multirow[t]{2}{*}{ P-value - } \\
\hline & $\mathrm{N}$ & $(\%)$ & $\bar{N}$ & $(\%)$ & \\
\hline \multicolumn{6}{|c|}{ Duration until first infection } \\
\hline mean, days & 45.1 & & 27.8 & & $0.109 * *$ \\
\hline range, days & 384 & & 141 & & \\
\hline SD, days & 59.4 & & 25.8 & & \\
\hline \multicolumn{6}{|c|}{ Neutros first infection } \\
\hline count & 1.37 & & 0.54 & & $0.654 * *$ \\
\hline range & 9.30 & & 3.40 & & \\
\hline
\end{tabular}

*includes probable and definite CVC-related bloodstream infections

**Mann-Whitney $-U$ Test used

Significantly more CVC-BSI with Gram-negative rods were found in the heparin group (7) than in the TSC group ( $1 ; \mathrm{P}=0.041$ ). Of the Gram-negative rods 63\% was Escherichia coli, and 3 of 5 Escherichia coli were also present in cultures from feces (table 4). $50 \%$ of patients with a Gram-negative CVC-BSI was non-neutropenic and did not get ciprofloxacin as selective decontamination of the digestive tract at the time of infection.

Seventeen patients developed more than one CVC-BSI ( 8 in the heparin group and 9 in the TSC group).

An incidence of $60 \%$ of CVC-BSI was seen in patients treated with intensive chemotherapy compared to only $7 \%$ of patients undergoing an autologous stem cell transplantation. This was well balanced between both arms.

Table 4a. Organisms causing CVC-BSI

\begin{tabular}{llcl}
\hline Organism & Heparin group & Citrate group & $P$-value \\
\hline CNS; S. epi; S hemolyticus & 15 & 17 & \\
S aureus & 3 & 0 & \\
Streptococcus $^{*}$ & 6 & 14 & \\
Gram neg $^{* *}$ & 7 & 1 & 0.041 \\
Other $^{*}$ & 3 & 3 & \\
\hline
\end{tabular}

*enterococcus, S. mitis, S. bovis, S. viridans, S.oralis

** different species, pantoea, corynebacterium, candida, clostridium

Table 4b. Organisms causing CVC-BSI with gram negative rods

\begin{tabular}{lll}
\hline Organism & Heparin group & Citrate group \\
\hline Escherichia Coli & 5 & 0 \\
Pseudomonas aeruginosa & 1 & 0 \\
Proteus mirablis & 1 & 0 \\
Stenotrophomonas maltophilia & 0 & 1 \\
\hline
\end{tabular}


Secondary endpoints: safety, adverse events and bleeding episodes

No serious adverse events that could be contributed to the locking solution were reported in both groups.

Two thrombocytopenic patients in the TSC group were reported to have a severe bleeding probably unrelated to the locking solution. One patient had a gastrointestinal bleeding and one patient had a bleeding in the thalamus. The bleeding in the thalamus was caused by a CNS localization of a Burkitt lymphoma. Twelve patients in the heparin group and seven patients in the TSC group died while having the CVC in place.

We were not able to report hypocalcemic symptoms accurately since most patients already had altered sensations in their mouth.

\section{DISCUSSION}

This is the first randomized controlled study that evaluated the efficacy of TSC as a locking solution compared to heparin in preventing CVC-related infections and thrombosis in patients with hematological malignancies undergoing intensive chemotherapy.

TSC had been shown to be an effective antimicrobial catheter locking solution able to significantly reduce CVC-related infections in hemodialysis patients $[15,16]$.

We found no difference in the incidence of probable and definite CVC-BSI with heparin locking and TSC locking. The incidence of CVC-BSI we found seems higher than the incidences observed in other studies with hematological patients [6, 9, 20-22]. When comparing the incidences of CVC-related bloodstream infections per 1000 catheter days the differences are less marked. The dwelling time of our CVCs is much longer than in most studies reported. Chee et all already demonstrated a continuous risk of CVC-related BSI with time in situ, without any obvious fall in risk in those CVCs in situ for prolonged periods [23].

In our study almost 50\% of the included patients underwent intensive chemotherapy and as shown in our study $60 \%$ of patients undergoing intensive chemotherapy developed a CVC-related bloodstream infection.

However, incidence rates of catheter infections can only be compared with caution, owing to different definitions, heterogeneous patient groups and different methods of prophylaxis against infections.

CNS were detected as the causative pathogen in most episodes in both the heparin and TSC group. Unexpectedly, we found seven times more CVC-BSI with Gram negative organisms in the heparin group than in the TSC group. Since the majority of CVCs with Gram-negative infections have to be removed due to hemodynamic instability or persistence of fevers and/or positive blood cultures despite broad-spectrum antibiotic cover this is of clinical relevance [23]. BSI with gram negative rods probably origin from 
translocation from the gastrointestinal tract. An explanation may be that TSC is superior to heparin in eradication of gram-negative rods in existing biofilms. This is supported by the finding that in vitro total elimination of Escherichia coli in biofilms has been shown with $7 \%$ citrate while heparin only had a minimal effect [24].

The incidences of asymptomatic and symptomatic CVC-related thrombosis are almost the same as seen in most studies [21, 25-28]. Comparing incidences is difficult since the reported incidence of CVC-related thrombosis varies considerably and has decreased over time. The lowering incidence is most likely the result of improved central venous catheters and to the better insertion procedures with the use of ultrasound.

A few promising studies have been published on the use of TSC as a locking agent in hemodialysis patients. The use of TSC lowered the incidence of CVC-BSI in hemodialysis patients from 4.1 CVC-BSI per 1000 catheter days to 1.1 per 1000 catheter days [16]. The reason we did not see statistically significant differences on BSI with CNS can be explained in several ways. The most important explanation is that alternative sources of bacteremia like microbial translocation during chemotherapy-induced mucositis have been important as well in our patients compared to hemodialysis patients. Another important explanation is the severe immunocompromised character of our patient population with a long duration of neutropenia. Finally in hematological patients CVCS are used very intensively for administering chemotherapy, parenteral nutrition, blood products as well for taking blood samples several times a day. In hemodialysis patients the CVC is only used two-three times a week for dialysis.

Some aspects of our study warrant comment. Firstly there was a premature stop of the study. Because the inclusion rate was lower than expected an interim analysis after the inclusion of 212 patients, blinded for the investigators, was performed. Based on the obtained results it was not anticipated that continuing the study would essentially alter the findings of the study. However we did include 212 patients of the planned accrual of 260 patients, have a long period of prospective follow up and not many missing data.

Secondly our study was not blinded. Reason we did not blind our study was the hypocalcemic symptoms and sensation in the mouth would never blind our study for $100 \%$ in practice.

Thirdly we did not record the duration of locking days and the duration of no-lock use. Since both arms are well balanced we do not expect differences between them that would affect the outcomes of the study.

In conclusion, this study does show a statistically significant reduction of CVC-BSI with Gram-negative rods in the citrate group which is a clinically relevant finding and warrants further investigation. However, no differences in CVC-BSI with gram positives and/or CVC-related thrombosis were seen. 


\section{REFERENCES}

1. McGee DC, Gould MK. Preventing complications of central venous catheterization. N Engl J Med 2003; 348: 1123-1133.

2. van Rooden CJ, Schippers EF, Barge RM et al. Infectious complications of central venous catheters increase the risk of catheter-related thrombosis in hematology patients: a prospective study. J Clin Oncol 2005; 23: 2655-2660.

3. Raad, II, Luna M, Khalil SA et al. The relationship between the thrombotic and infectious complications of central venous catheters. Jama 1994; 271: 1014-1016.

4. Lordick F, Hentrich $M$, Decker T et al. Ultrasound screening for internal jugular vein thrombosis aids the detection of central venous catheter-related infections in patients with haemato-oncological diseases: a prospective observational study. Br J Haematol 2003; 120: 1073-1078.

5. Cortelezzi A, Moia M, Falanga A et al. Incidence of thrombotic complications in patients with haematological malignancies with central venous catheters: a prospective multicentre study. $\mathrm{Br} J$ Haematol 2005; 129: 811-817.

6. Boersma RS, Jie KS, Verbon A et al. Thrombotic and infectious complications of central venous catheters in patients with hematological malignancies. Ann Oncol 2008; 19: 433-442.

7. Lee $A Y$, Kamphuisen PW. Epidemiology and prevention of catheter-related thrombosis in patients with cancer. J Thromb Haemost 2012; 10: 1491-1499.

8. Mehall JR, Saltzman DA, Jackson RJ, Smith SD. Fibrin sheath enhances central venous catheter infection. Crit Care Med 2002; 30: 908-912.

9. van Rooden CJ, Schippers EF, Guiot HF et al. Prevention of coagulase-negative staphylococcal central venous catheter-related infection using urokinase rinses: a randomized double-blind controlled trial in patients with hematologic malignancies. J Clin Oncol 2008; 26: 428-433.

10. Abdelkefi A, Torjman L, Ladeb $S$ et al. Randomized trial of prevention of catheter-related bloodstream infection by continuous infusion of low-dose unfractionated heparin in patients with hematologic and oncologic disease. J Clin Oncol 2005; 23: 7864-7870.

11. Boersma RS, Schouten HC. Clinical practices concerning central venous catheters in haematological patients. Eur J Oncol Nurs 2010; 14: 200-204.

12. Mitchell MD, Anderson BJ, Williams K, Umscheid CA. Heparin flushing and other interventions to maintain patency of central venous catheters: a systematic review. J Adv Nurs 2009; 65: 2007-2021.

13. Karaaslan $H$, Peyronnet $P$, Benevent $D$ et al. Risk of heparin lock-related bleeding when using indwelling venous catheter in haemodialysis. Nephrol Dial Transplant 2001; 16: 2072-2074.

14. Weijmer MC, Debets-Ossenkopp YJ, Van De Vondervoort FJ, ter Wee PM. Superior antimicrobial activity of trisodium citrate over heparin for catheter locking. Nephrol Dial Transplant 2002; 17: 2189-2195.

15. Winnett G, Nolan J, Miller M, Ashman N. Trisodium citrate $46.7 \%$ selectively and safely reduces staphylococcal catheter-related bacteraemia. Nephrol Dial Transplant 2008; 23: 3592-3598.

16. Weijmer MC, van den Dorpel MA, Van de Ven PJ et al. Randomized, clinical trial comparison of trisodium citrate $30 \%$ and heparin as catheter-locking solution in hemodialysis patients. J Am Soc Nephrol 2005; 16: 2769-2777.

17. Niyyar VD. Catheter dysfunction: the role of lock solutions. Semin Dial 2012; 25: 693-699.

18. Mermel LA, Allon M, Bouza E et al. Clinical practice guidelines for the diagnosis and management of intravascular catheter-related infection: 2009 Update by the Infectious Diseases Society of America. Clin Infect Dis 2009; 49: 1-45.

19. Baxter GM, Kincaid W, Jeffrey RF et al. Comparison of colour Doppler ultrasound with venography in the diagnosis of axillary and subclavian vein thrombosis. Br J Radiol 1991; 64: 777-781.

20. Slobbe L, Doorduijn JK, Lugtenburg PJ et al. Prevention of catheter-related bacteremia with a daily ethanol lock in patients with tunnelled catheters: a randomized, placebo-controlled trial. PLoS One 2010;5: e10840. 
21. Abdelkefi A, Ben Othman $T$, Kammoun $L$ et al. Prevention of central venous line-related thrombosis by continuous infusion of low-dose unfractionated heparin, in patients with haemato-oncological disease. A randomized controlled trial. Thromb Haemost 2004; 92: 654-661.

22. Ruschulte H, Franke M, Gastmeier P et al. Prevention of central venous catheter related infections with chlorhexidine gluconate impregnated wound dressings: a randomized controlled trial. Ann Hematol 2009; 88: $267-272$.

23. Chee L, Brown M, Sasadeusz J et al. Gram-negative organisms predominate in Hickman line-related infections in non-neutropenic patients with hematological malignancies. J Infect 2008; 56: 227-233.

24. Steczko J, Ash SR, Nivens DE et al. Microbial inactivation properties of a new antimicrobial/antithrombotic catheter lock solution (citrate/methylene blue/parabens). Nephrol Dial Transplant 2009; 24: 1937-1945.

25. Verso M, Agnelli G. Venous thromboembolism associated with long-term use of central venous catheters in cancer patients. J Clin Oncol 2003; 21: 3665-3675.

26. Verso M, Agnelli G, Bertoglio $S$ et al. Enoxaparin for the prevention of venous thromboembolism associated with central vein catheter: a double-blind, placebo-controlled, randomized study in cancer patients. J Clin Oncol 2005; 23: 4057-4062.

27. Niers TM, Di Nisio M, Klerk CP et al. Prevention of catheter-related venous thrombosis with nadroparin in patients receiving chemotherapy for hematologic malignancies: a randomized, placebo-controlled study. J Thromb Haemost 2007; 5: 1878-1882.

28. Lee $A Y$, Levine $M N$, Butler $G$ et al. Incidence, risk factors, and outcomes of catheter-related thrombosis in adult patients with cancer. J Clin Oncol 2006; 24: 1404-1408. 



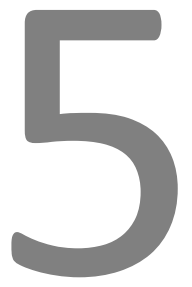

Congenital thrombophilia

and central venous

catheter-related thrombosis

in patients with cancer

R.S. Boersma, K. Hamulyak, H. ten Cate, H.C. Schouten

Clin Appl Thromb Hemost. 2010 Dec;16(6):643-649
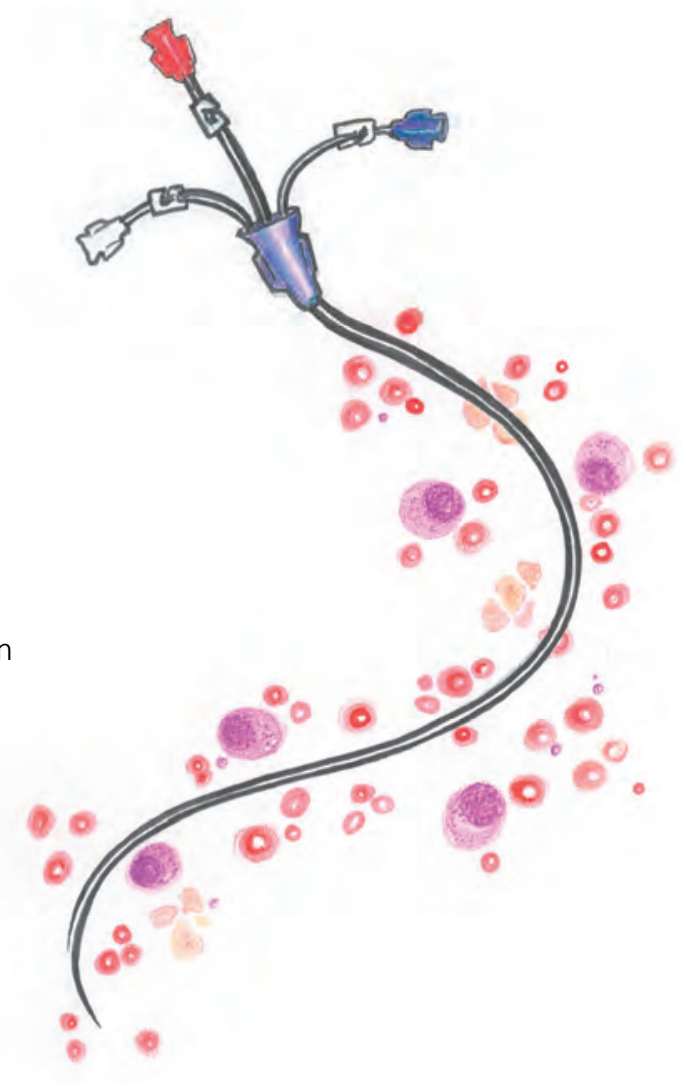


\section{ABSTRACT}

Central venous catheters (CVCs) have considerably improved the management of cancer patients. Reported complications of insertion consist of mechanical complications during the insertion and long-term complications such as CVC-related thrombosis and infections. CVC-related thrombosis is a frequently occurring complication and may cause significant morbidity in patients with cancer.

Congenital thrombophilia has been shown to increase the risk of deep vein thrombosis of the leg and pulmonary embolism in cancer patients.

The aim of this narrative review is to discuss the main studies that examined whether a state of thrombophilia increases the risk of CVC-related thrombosis in patients with cancer. The studies were retrieved by an extensive Medline search.

The identification of risk factors for CVC-related thrombosis would allow stratification of cancer patients into higher or lower risk groups, and targeting of prophylaxis to high-risk patients likely to benefit most.

Patients with cancer with a CVC and a factor V Leiden mutation have a higher risk of developing CVC-related thrombosis than cancer patients with a CVC without the mutation. The scarce information available suggests hyperhomocysteinemia to be a risk factor for CVC-related thrombosis. For other congenital thrombophilia factors like the G20210A mutation and the congenital deficiencies in protein C, protein $\mathrm{S}$ or antithrombin the available data are too limited to allow for any definitive conclusions to be made.

Since the clinical implications of all these findings remain to be clarified, routine screening of cancer patients with a CVC for thrombophilia cannot yet be recommended on the basis of the studies discussed. 


\section{INTRODUCTION}

Central venous catheters (CVCs) are frequently used in patients with hematological and oncological malignancies in order to administer chemotherapy, stem cell infusions, blood products, medication, and parenteral hyperalimentation, as well as for blood sampling.

The benefit derived from CVCs can be offset by complications. Reported complications consist of mechanical complications during or directly after the insertion (arterial puncture, hematoma and pneumothorax) and long term complications like CVC-related infections and thrombosis [1]. CVC-related infections and thrombosis result in patient morbidity, significant increases in the length of hospitalization and medical care costs [2, 3].

Two types of CVC-related thrombosis can be distinguished; symptomatic and asymptomatic thrombosis. Symptomatic CVC-related thrombosis is defined as thrombosis objectified by diagnostic imaging upon overt signs and symptoms, such as pain, swelling or edema. Asymptomatic CVC-related thrombosis is demonstrated by screening diagnostic imaging and without any signs or symptoms suggestive of CVC-related thrombosis. Asymptomatic thrombosis has a relative risk of 6.8 of developing a symptomatic CVCrelated thrombosis and increases the risk of CVC-related infections $[4,5]$.

In our review, published in 2008, we evaluated the incidence of long-term complications of CVCs in hematological patients. The incidence of symptomatic CVCrelated thrombosis in hematological adult patients varies between $1.2 \%$ and $18.0 \%$ and the incidence of asymptomatic CVC-related thrombosis varies between 1.5-34\% [6].

Complications of a CVC-related thrombosis are catheter dysfunction, post thrombotic syndrome, CVC-related infections and a pulmonary embolism. The reported incidence of a pulmonary embolism (PE) as a complication of CVC-related thrombosis varies. Monreal et al systematically screened 84 patients with a symptomatic CVC-related thrombosis for a PE with a ventilation-perfusion scan. They found a $15 \%$ cumulative incidence of PE [7].

Venous thromboembolism occurs commonly in patients with cancer and is the second leading cause of death in these patients [8]. Compared with patients without cancer, the risk of symptomatic venous thromboembolism (VTE) in patients with cancer is increased 4- to 7-fold [9, 10].

Even though the association between cancer and hypercoagulability is well established, the pathogenesis of VTE in these patients has not been entirely elucidated $[11,12]$. While hypercoagulability is encountered in up to $90 \%$ of cancer patients, only $4-15 \%$ of them develop VTE.

Besides hypercoagulability, other risk factors for VTE in cancer patients include longterm immobilization, surgery, insertion of central venous catheters and chemotherapy 
[13]. Chemotherapy increases the risk of VTE by damaging the vascular endothelium and decreasing plasma levels of naturally occurring coagulation inhibitors [14].

Congenital thrombophilia has been shown to increase the risk of thrombosis even more in cancer patients. In a large population-based case-control study by Blom et al, the risk of venous thromboembolism was increased 12 to 17 -fold in patients with both cancer and factor $V$ Leiden or the G20210A mutation compared to 7-fold in patients with cancer without factor $V$ Leiden or the G20210A mutation[9]. These data about congenital thrombophilia may be relevant in cancer patients who need to undergo central venous catheterization since they may indicate differences in thrombotic risk and may help predicting CVC-related thrombosis in the individual. It may also help to select high risk patients for antithrombotic prophylaxis

Anticoagulant prophylaxis has not been proven effective in reducing the incidence of CVC-related thrombosis [15-18]. Therefore, until effective regimens are identified, the American College of Chest Physicians (ACCP), the Italian Association of Medical Oncology (AIOM), the National Comprehensive Cancer Network (NCCN), and European Society of Medical Oncology (ESMO) guidelines do not recommend routine anticoagulant prophylaxis in order to prevent CVC-related thrombosis [8, 19-22].

The aim of this narrative review is to discuss the main studies that examined whether a state of congenital thrombophilia increases the risk of CVC-related thrombosis in patients with cancer. Since the plasma concentrations of proteins involved in hemostasis differ in children from those in adults and the significance of the risk factors may vary between adults and children, only studies involving adult cancer patients will be discussed.

An extensive literature review of the studies published between 1990 en January 2010 was performed using the MEDLINE database and the following subject headings: central venous catheter, central venous catheter-related thrombosis, thrombophilic abnormalities, Factor $V$ Leiden and the G20210A prothrombin mutation.

The literature search was limited to publications in English. References of all the articles were crosschecked to identify additional articles. We only included articles that assessed long-term CVCs in adult patients with a hematological or oncological malignancy.

\section{FACTOR V LEIDEN}

Inherited resistance to activated protein $C$ is a prothrombotic condition resulting from a gain-of-function mutation of coagulation factor $\mathrm{V}$, commonly referred to as factor $\mathrm{V}$ Leiden. A single guanine $(G)$ to adenine $(A)$ missense mutation in the factor $V$ gene at nucleotide 1691 substitutes $G$ for $A$, resulting in a mutant protein resistant to the anticoagulant action of activated protein $\mathrm{C}$ [23]. This mutation is the most common 
inherited risk factor for VTE, with a prevalence of $5 \%$ in the general population and 20$50 \%$ among patients with VTE. The estimated risk of deep venous thrombosis (DVT) in heterozygous carriers of the mutation is five-to 10-fold higher, whereas for homozygous carriers it is 80-to 100-fold higher, than in non-carriers [24].

The prevalence of factor $V$ Leiden in cancer patients ranges from 4.0-9.7\% with a relative risk of developing CVC-related thrombosis of 2.6-7.7 (see table 1).

Table 1. Prevalence of factor $V$ Leiden and risk estimates of CVC-related thrombosis in patients with cancer and the presence of Factor $V$ Leiden

\begin{tabular}{|c|c|c|c|c|c|}
\hline Author & Year & $\begin{array}{l}\text { Type of } \\
\text { Study }\end{array}$ & Patients & $\begin{array}{l}\text { Prevalence } \\
\text { FVL (\%) in } \\
\text { cohort studied }\end{array}$ & $\begin{array}{l}\text { Risk }(95 \% \mathrm{Cl}) \text { of CVC- } \\
\text { related thrombosis } \\
\text { in patients with } \\
\text { Factor } \mathrm{V} \text { Leiden }\end{array}$ \\
\hline Fijnheer et al & 2002 & Cohort & $\begin{array}{l}277 \text { hematological patients } \\
\text { undergoing HSCT }\end{array}$ & 4.8 & RR 7.7 (3.3-17.9) \\
\hline Ratcliffe et al & 1999 & Cohort & $\begin{array}{l}84 \text { patients with solid tumors and } \\
\text { hematological malignancies }\end{array}$ & 4.0 & ND \\
\hline Van Rooden et al & 2004 & Cohort & $\begin{array}{l}252 \text { patients: } 38.5 \% \text { hematological } \\
\text { pts, } 15.5 \% \text { solid tumors }\end{array}$ & 6.7 & RR 2.6 (1.8-3.8) \\
\hline Abdelkefi et al & 2005 & Cohort & $\begin{array}{l}171 \text { hematological patients } \\
\text { undergoing HSCT }\end{array}$ & 5.8 & RR 4.8 (1.5-14.8) \\
\hline Tesselaar et al* & 2004 & Cohort & $\begin{array}{l}101 \text { patients with solid tumors } \\
\text { receiving chemotherapy }\end{array}$ & 9.7 & OR $0.6(0.1-5.5)$ \\
\hline Mandala et al & 2004 & $\begin{array}{l}\text { Case } \\
\text { control }\end{array}$ & $\begin{array}{l}75 \text { breast cancer patients receiving } \\
\text { fluorouracil-based chemotherapy }\end{array}$ & 9.3 & OR 6.1 (1.1-34.3) \\
\hline
\end{tabular}

$\mathrm{FVL}=$ factor $\mathrm{V}$ Leiden

$\mathrm{ND}=$ not determined; $\mathrm{Cl}=$ confidence interval; $\mathrm{OR}=$ odds ratio; $\mathrm{RR}$ = relative risk

$\mathrm{HSCT}=$ hematopoietic stem cell transplantation

* patients with Factor V Leiden or prothrombin G20210A mutation were included in this analysis

Abdelkefi et al showed in a well performed prospective study in 171 hematopoietic stem cell transplants that (a)symptomatic CVC-related thrombosis was diagnosed in three out of 10 patients (30\%) with factor $V$ Leiden as compared to 10 of 161 patients (6.2\%) who did not carry the mutation (RR 4.8; Cl 95\% 1.5-14.8) [25].

In a study of 277 patients undergoing bone marrow transplantation, a 54\% frequency of clinically manifest thrombosis (7 out of 13 patients) in patients who were heterozygous for factor $\mathrm{V}$ Leiden was reported, whereas in patients without the factor $\mathrm{V}$ Leiden mutation a $10 \%$ risk was found (26 of 264 patients). The reported relative risk was 7.7 (Cl 95\%; 3.3-17.9) [26].

Van Rooden et al showed in a prospective study in 252 patients with a CVC (only $54 \%$ of patients had a malignant disease) a cumulative incidence of (a)symptomatic CVC-related thrombosis of $30 \%$ (clinically manifest $7 \%$ ). Thrombosis was diagnosed in 
12 of the 17 patients with factor $\vee$ Leiden (70.6\%), as compared to 63 of the 235 patients who did not have the mutation (26.8\%) (RR 2.6, CI 95\% 1.8 to 3.8) [27].

Tesselaar et al performed a prospective study in 101 cancer patients with implantable ports undergoing chemotherapy. Eighteen of these patients (18\%) developed symptomatic CVC-related thrombosis. Only one of these eighteen patients had a factor $V$ Leiden or prothrombin G20210a mutation compared to 7 out of the 83 patients in the group without evidence of CVC-related thrombosis (NS).

In a smaller study in which 82 cancer patients with a CVC were evaluated, prothrombotic risk factors, including factor $V$ Leiden, were not substantial predictors of clinically manifest thrombosis, although the data suggested that factor $V$ Leiden increased the risk of thrombosis. However, the statistical power of this study was limited because of the small numbers of patients with thrombosis and factor $V$ Leiden [28].

Mandala et al studied 300 patients with locally advanced or metastatic breast cancer. 25 patients (8.3\%) who developed a CVC-related thrombosis and 50 controls (patients with breast cancer and a CVC without evidence of CVC-related thrombosis) were included in a nested case-control study. Five cases and two controls were heterozygous carriers of the factor $V$ Leiden mutation. The age-adjusted odds ratio for CVC-related thrombosis was 6.1 (95\% Cl 1.1-34.3) [29].

In one other case-control study it was reported that factor $V$ Leiden did not contribute to CVC-related thrombosis. However, a relative small number of patients was studied (27), while the presence of factor $V$ Leiden in patients who did not develop CVCrelated thrombosis was not assessed. This case-control study also did not observe a higher prevalence of factor $V$ Leiden in patients with thrombosis compared to the general population [30].

It is known that the presence of a concomitant inherited thrombophilic defect in carriers of factor $V$ Leiden further increases the risk of a first thrombotic event[31-33]. No data on this subject are published concerning cancer patients and CVC-related thrombosis.

Reviewing only the prospective cohort studies with at least 100 patients included, it appears that patients with cancer with a CVC and a factor $V$ Leiden mutation have a significant higher risk (RR 2.6-7.7) of developing CVC-related thrombosis than cancer patients with a CVC without the mutation.

\section{G20210A PROTHROMBIN MUTATION}

The G20210A prothrombin gene gain-of function mutation has been identified in the 3 'untranslated region of the prothrombin gene (the substitution of $A$ for $G$ at position 20210) and is associated with high plasma levels of factor II. 
The mutant allele is present in $2 \%$ of the general population and in $6 \%$ in unselected patients with a first venous thromboembolic event and increases the risk of VTE by three-to five-fold [24].

The prevalence of the prothrombin G20210A mutation in cancer patients ranges between 0.6-2.6 with a relative risk of developing CVC-related thrombosis of $2.3(95 \% \mathrm{Cl}$ 1.3-4.2) (see table 2).

Table 2. Prevalence of the G20210A mutation and risk estimates of CVC-related thrombosis in patients with cancer and the G20210A prothrombin mutation

\begin{tabular}{|c|c|c|c|c|c|}
\hline Author & Year & $\begin{array}{l}\text { Type of } \\
\text { Study }\end{array}$ & Patients & $\begin{array}{l}\text { Prevalence (\%) } \\
\text { in the cohort } \\
\text { studied }\end{array}$ & $\begin{array}{l}\text { Risk }(95 \% \mathrm{Cl}) \text { of } \\
\text { CVC-related } \\
\text { thrombosis in } \\
\text { patients with the } \\
\text { G20210A mutation }\end{array}$ \\
\hline Abdelkefi et al & 2004 & Cohort & $\begin{array}{l}171 \text { hematological patients } \\
\text { undergoing HSCT }\end{array}$ & 0.6 & ND \\
\hline Van Rooden et al & 2004 & Cohort & $\begin{array}{l}252 \text { pts: } 38.5 \% \text { hematological } \\
\text { patients, } 15.5 \% \text { solid tumors }\end{array}$ & 2.4 & RR 2.3 (1.3-4.2) \\
\hline Mandala et al & 2004 & Cohort & $\begin{array}{l}75 \text { breast cancer patients receiving } \\
\text { fluorouracil-based chemotherapy }\end{array}$ & 2.6 & ND \\
\hline
\end{tabular}

HSCT = hematopoietic stem cell transplantation

$\mathrm{Cl}=$ confidence interval; $\mathrm{RR}=$ relative risk

ND $=$ not determined

Van Rooden et al performed a prospective study assessing the incidence of factor $V$ Leiden and the prothrombin G20210A mutation to CVC-related thrombosis. They included 252 patients of whom $15.5 \%$ had a solid tumour and $38.5 \%$ a hematological malignancy. CVC-related thrombosis was diagnosed in 4 of the 6 patients (66.7\%) with the prothrombin G20210A mutation, whereas 71 CVC-related thromboses were detected in 246 patients (28.9\%) without the mutation (RR 2.3, Cl 95\% 1.3 - 4.2) [27].

Abdelkefi et al determined the incidence of inherited prothrombotic abnormalities in blood coagulation to CVC-related thrombosis in 171 hematopoietic stem cell transplant recipients. There was only one hematopoietic stem cell transplant patient with the prothrombin G20210A mutation which does not allow us to make conclusions [25].

In 75 patients with breast cancer and a CVC studied by Mandala et al, only one patient with a symptomatic CVC-related thrombosis carried the G20210A mutation in the prothrombin gene. No patients in the control group (patients with breast cancer with a CVC without a symptomatic CVC-related thrombosis) carried the mutation ( $p=0.30)$ [29] .

Concluding, there is not sufficient information in the literature about the prothrombin G20210A mutation and CVC-related thrombosis in cancer patients and no definitive 
conclusions can be made. The study from van Rooden suggests the prothrombin G20210A mutation (RR 2.3) to be associated with CVC-related thrombosis in cancer patients.

\section{PROTEIN C DEFICIENCY, PROTEIN S DEFICIENCY AND ANTITHROMBIN DEFICIENCY}

Congenital deficiencies in protein $\mathrm{C}$, protein S or antithrombin, three core inhibitors of the coagulation cascade, substantially increase the risk of VTE. These deficiencies are rare and detectable only in less than $1 \%$ of the general population.

Abdelkefi et al diagnosed an (a)symptomatic CVC-related thrombosis in one out of five (20\%) hematopoietic stem cell patients with protein C deficiency, whereas 12 thromboses were detected in the other 166 hematopoietic stem cell transplant patients (7.2\%) without protein C deficiency.

Three patients had evidence of protein S deficiency and only one patient had evidence of antithrombin deficiency. In these last four patients no CVC-related thrombosis was seen [25].

Concluding as these deficiencies are rare and hence the information available is scarce, their potential role on the occurrence of CVC-related thrombosis in cancer patients is unknown.

\section{HYPERHOMOCYSTEINEMIA}

Homocysteine is an amino acid formed in the methionine metabolism. Dietary deficiencies of folate, vitamin B12, and B6 as well as inherited enzyme defects (e.g. methylenetetrahydrofolate reductase (MTHFR) gene polymorphisms) could lead to increases in homocysteine plasma concentration [34, 35]. Hyperhomocysteinemia is an established risk factor for arterial and venous thrombosis, atherosclerosis and vascular disease. In the general population, this abnormality is associated with a two-to threefold increase in the risk of venous thromboembolism. The relationship between thrombophilia and a mutation in the MTHFR C677T which is associated to a thermolabile phenotype, decreased enzyme activity and mild hyperhomocysteinemia is still controversial [36].

Patients with solid tumors or hematological malignancies exhibit higher plasma levels of homocysteine [37, 38].

Melillo et al found hyperhomocysteinemia to occur in $46 \%$ of patients with acute leukemia. The incidence of venous thromboembolism was 9.6\%. In multivariate analysis, hyperhomocysteinemia was the only risk factor for venous thromboembolism (OR 33.90; $95 \% \mathrm{Cl} 1.53-751.33 ; \mathrm{p}=0.026)$. No specifications concerning the percentage of CVC-related thrombosis are made in this study [37]. 
In one study of 101 cancer patients with a CVC 22 patients (21.7\%) had elevated plasma homocysteine levels. The odds ratio for CVC-related thrombosis was 3.8 (95\% confidence interval: 1.3-11.3) in patients with elevated plasma levels of homocysteine, compared with those with normal plasma levels of homocysteine. Of note, in this study, neither factor $V$ Leiden nor G20210A prothrombin mutation was associated with CVCrelated thrombosis [39].

There are various possible reasons for the raised total plasma homocysteine levels (tHcy) levels in patients with cancer. Total plasma homocysteine may be increased because of the relative low red cell folate as a result of poor intake in the presence of malignancy. Another explanation could be that raised homocysteine is related to the cancer itself and the extent of disease [34, 35]. Gatt et al demonstrated significantly higher levels of homocysteine in patients with metastatic breast carcinoma compared to patients with early breast cancer. As more homocysteine is generated, this could act as a toxin to the endothelium increasing the risk of thrombosis in these patients. This observation could explain the high rate of venous thrombosis and CVC-related thrombosis in patients with metastatic disease [34, 40].

Concluding patients with a malignant disease exhibit higher plasma levels of homocysteine compared to the general population. The limited evidence available suggests a higher risk of CVC-related thrombosis in patients with a CVC and elevated plasma levels of homocysteine.

\section{ANTIPHOSPHOLIPID ANTIBODIES}

Antiphospholipid antibody syndrome is characterized clinically by venous or arterial thrombosis, recurrent fetal loss, or placental insufficiency in women. The diagnosis requires the persistent laboratory evidence of antibodies to phospholipids or plasma proteins that have affinity to the negatively charged phospholipids [41]. Antiphospholipid antibodies also play a role in the thrombotic predisposition of malignancy. There are several possible theories as to the generation of antiphospholipid antibodies in the setting of malignancy: production of auto antibodies by the immune system in response to tumor antigens, production of monoclonal antibodies with lupus anticoagulant and anticardiolipin activities and secretion of anticardiolipin activity from tumor cells $[42,43]$.

The incidence of antiphospholipid antibodies and the diagnosis of antiphospholipid syndrome may be much higher in patients who have cancer. An Italian study of 100 consecutive patients who had lymphoma indicated that 27 patients tested positive for either anticardiolipin antibody or lupus anticoagulant [44]. This was much higher than the $8 \%$ prevalence seen in their age-and sex-matched control group. If restricted to patients who have malignancy and thrombosis, antiphospholipid antibodies may be seen in up to $60 \%$ of patients according to one study of Asian cancer patients $[43,45]$. It 
remains to be determined whether having antiphospholipid antibodies in the setting of malignancy represents an epiphenomenon or indeed leads to a higher risk of thrombosis.

No data about antiphospholipid bodies and CVC-related thrombosis are presently published.

\section{PERSONAL OR FAMILY HISTORY OF VENOUS THROMBOEMBOLISM}

A positive family history for venous thrombosis increases the risk of venous thrombosis in a general population regardless of the risk factors precipitating the thrombosis (OR 2.2; $\mathrm{Cl} 1.9-2.6)[46]$.

In a prospective study Van Rooden et all assessed risk factors for CVC-related thrombosis. They found a personal history of venous thrombosis to be associated with an increased risk of CVC-related thrombosis (RR 2.0; CI 95\% 1.3-3.0). A family history of venous thromboembolism gave a relative risk of developing CVC-related thrombosis of 1.3 (Cl 95\% 0.8-2.2) [27].

\section{CONCLUSION}

CVC-related thrombosis is a common and severe complication in patients with cancer. The identification of risk factors for this severe event would allow stratification of cancer patients into higher or lower risk groups, and targeting of prophylaxis to high-risk patients likely to benefit most.

We reviewed studies assessing whether congenital thrombophilia influenced the risk of CVC-related thrombosis in cancer patients. In contrast to a large number of studies on the association of congenital thrombophilia with DVT of the leg and pulmonary embolism, studies investigating the association between thrombophilia and CVC-related thrombosis in cancer patients are scarce.

As expected, the prevalence and reported risk estimates of factor $V$ Leiden or the G20210A mutation in cancer patients are not higher than in the general population. Patients with cancer exhibit higher plasma levels of homocysteine. Patients with cancer and the presence of a CVC and a factor $V$ Leiden mutation have a higher risk of developing CVC-related thrombosis than cancer patients with a CVC without the mutation. There is limited information available about hyperhomocysteinemia and the risk of CVC-related thrombosis. The information available suggests hyperhomocysteinemia to be a risk factor for CVC-related thrombosis.

For the other above described congenital thrombophilia factors the available data are too limited to allow for any definitive conclusions to be made. The majority of studies were of limited size resulting in wide confidence intervals for risk estimates. In 
addition, it is possible that due to the cancer associated risk of thrombosis the contributing effect of any congenital mutations may be too low to become apparent as is likely to be the case in the study of Tesselaar [39].

Since the clinical implications of all these findings remain to be clarified, routine screening or screening of specific groups of cancer patients with a CVC for thrombophilia cannot yet be recommended on the basis of the studies discussed. 


\section{REFERENCES}

1. McGee DC, Gould MK. Preventing complications of central venous catheterization. N Engl J Med 2003; 348: 1123-1133.

2. Wisplinghoff $\mathrm{H}$, Cornely OA, Moser $\mathrm{S}$ et al. Outcomes of nosocomial bloodstream infections in adult neutropenic patients: a prospective cohort and matched case-control study. Infect Control Hosp Epidemiol 2003; 24: 905-911.

3. Wenzel RP, Edmond MB. The impact of hospital-acquired bloodstream infections. Emerg Infect Dis 2001; 7: $174-177$.

4. Lordick F, Hentrich M, Decker T et al. Ultrasound screening for internal jugular vein thrombosis aids the detection of central venous catheter-related infections in patients with haemato-oncological diseases: a prospective observational study. Br J Haematol 2003; 120: 1073-1078.

5. van Rooden CJ, Rosendaal FR, Barge RM et al. Central venous catheter related thrombosis in haematology patients and prediction of risk by screening with Doppler-ultrasound. Br J Haematol 2003; 123: 507-512.

6. Boersma RS, Jie KS, Verbon A et al. Thrombotic and infectious complications of central venous catheters in patients with hematological malignancies. Ann Oncol 2008; 19: 433-442.

7. Monreal M, Raventos A, Lerma R et al. Pulmonary embolism in patients with upper extremity DVT associated to venous central lines--a prospective study. Thromb Haemost 1994; 72: 548-550.

8. Hirsh J, Guyatt G, Albers GW et al. Antithrombotic and thrombolytic therapy: American College of Chest Physicians Evidence-Based Clinical Practice Guidelines (8th Edition). Chest 2008; 133: 110S-112S.

9. Blom JW, Doggen CJ, Osanto S, Rosendaal FR. Malignancies, prothrombotic mutations, and the risk of venous thrombosis. Jama 2005; 293: 715-722.

10. Heit JA, Silverstein MD, Mohr DN et al. Risk factors for deep vein thrombosis and pulmonary embolism: a population-based case-control study. Arch Intern Med 2000; 160: 809-815.

11. Falanga A, Barbui T, Rickles FR, Levine MN. Guidelines for clotting studies in cancer patients. For the Scientific and Standardization Committee of the Subcommittee on Haemostasis and Malignancy International Society of Thrombosis and Haemostasis. Thromb Haemost 1993; 70: 540-542.

12. Falanga A, Marchetti M. Venous thromboembolism in the hematologic malignancies. J Clin Oncol 2009; 27: 4848-4857.

13. Prandoni P, Falanga A, Piccioli A. Cancer and venous thromboembolism. Lancet Oncol 2005; 6: $401-410$.

14. Lee AY, Levine MN. The thrombophilic state induced by therapeutic agents in the cancer patient. Semin Thromb Hemost 1999; 25: 137-145.

15. Couban S, Goodyear M, Burnell M et al. Randomized placebo-controlled study of low-dose warfarin for the prevention of central venous catheter-associated thrombosis in patients with cancer. J Clin Oncol 2005; 23: 4063-4069.

16. Verso M, Agnelli G, Bertoglio S et al. Enoxaparin for the prevention of venous thromboembolism associated with central vein catheter: a double-blind, placebo-controlled, randomized study in cancer patients. J Clin Oncol 2005; 23: 4057-4062.

17. Niers TM, Di Nisio M, Klerk CP et al. Prevention of catheter-related venous thrombosis with nadroparin in patients receiving chemotherapy for hematologic malignancies: a randomized, placebo-controlled study. J Thromb Haemost 2007; 5: 1878-1882.

18. Karthaus M, Doellmann $T$, Klimasch $T$ et al. Central venous catheter infections in patients with acute leukemia. Chemotherapy 2002; 48: 154-157.

19. Wagman LD, Baird MF, Bennett CL et al. Venous thromboembolic disease. Clinical practice guidelines in oncology. J Natl Compr Canc Netw 2006; 4: 838-869.

20. Mandala M, Falanga A, Roila F. Management of venous thromboembolism in cancer patients: ESMO clinical recommendations. Ann Oncol 2008; 19 Suppl 2: ii126-127.

21. Mandala M, Falanga A, Piccioli A et al. Venous thromboembolism and cancer: guidelines of the Italian Association of Medical Oncology (AIOM). Crit Rev Oncol Hematol 2006; 59: 194-204. 
22. Khorana AA, Streiff MB, Farge D et al. Venous thromboembolism prophylaxis and treatment in cancer: a consensus statement of major guidelines panels and call to action. J Clin Oncol 2009; 27: 4919-4926.

23. Bertina RM, Koeleman BP, Koster $T$ et al. Mutation in blood coagulation factor $V$ associated with resistance to activated protein C. Nature 1994; 369: 64-67.

24. Seligsohn U, Lubetsky A. Genetic susceptibility to venous thrombosis. N Engl J Med 2001; 344: 1222-1231.

25. Abdelkefi A, Ben Romdhane N, Kriaa A et al. Prevalence of inherited prothrombotic abnormalities and central venous catheter-related thrombosis in haematopoietic stem cell transplants recipients. Bone Marrow Transplant 2005; 36: 885-889.

26. Fijnheer R, Paijmans B, Verdonck LF et al. Factor $V$ Leiden in central venous catheter-associated thrombosis. Br J Haematol 2002; 118: 267-270.

27. Van Rooden CJ, Rosendaal FR, Meinders AE et al. The contribution of factor $V$ Leiden and prothrombin G20210A mutation to the risk of central venous catheter-related thrombosis. Haematologica 2004; 89: 201-206.

28. Ratcliffe M, Broadfoot $C$, Davidson $M$ et al. Thrombosis, markers of thrombotic risk, indwelling central venous catheters and antithrombotic prophylaxis using low-dose warfarin in subjects with malignant disease. Clin Lab Haematol 1999; 21: 353-357.

29. Mandala M, Curigliano G, Bucciarelli P et al. Factor V Leiden and G20210A prothrombin mutation and the risk of subclavian vein thrombosis in patients with breast cancer and a central venous catheter. Ann Oncol 2004; 15: 590-593.

30. Riordan M, Weiden PL. Factor $\mathrm{V}$ Leiden mutation does not account for central venous catheter-related thrombosis. Am J Hematol 1998; 58: 150-152.

31. Samama MM, Dahl OE, Quinlan DJ et al. Quantification of risk factors for venous thromboembolism: a preliminary study for the development of a risk assessment tool. Haematologica 2003; 88: 1410-1421.

32. Meinardi JR, Middeldorp S, de Kam PJ et al. Risk of venous thromboembolism in carriers of factor $V$ Leiden with a concomitant inherited thrombophilic defect: a retrospective analysis. Blood Coagul Fibrinolysis 2001; 12: 713-720.

33. Emmerich J, Rosendaal FR, Cattaneo $M$ et al. Combined effect of factor $V$ Leiden and prothrombin 20210A on the risk of venous thromboembolism--pooled analysis of 8 case-control studies including 2310 cases and 3204 controls. Study Group for Pooled-Analysis in Venous Thromboembolism. Thromb Haemost 2001; 86: 809-816.

34. Gatt A, Makris A, Cladd H et al. Hyperhomocysteinemia in women with advanced breast cancer. Int J Lab Hematol 2007; 29: 421-425.

35. Ruud E, Holmstrom H, Brosstad F, Wesenberg F. Children with acute lymphoblastic leukaemia have high plasma levels of total homocysteine at time of diagnosis. Scand J Clin Lab Invest 2006; 66: 67-78.

36. Mandala $M$, Barni S, Prins $M$ et al. Acquired and inherited risk factors for developing venous thromboembolism in cancer patients receiving adjuvant chemotherapy: a prospective trial. Ann Oncol 2010; 21 (4): 871-6..

37. Melillo L, Grandone E, Colaizzo D et al. Symptomatic venous thromboembolism and thrombophilic status in adult acute leukemia: a single-center experience of 114 patients at diagnosis. Acta Haematol 2007; 117: $215-220$.

38. Samonakis DN, Koutroubakis IE, Sfiridaki A et al. Hypercoagulable states in patients with hepatocellular carcinoma. Dig Dis Sci 2004; 49: 854-858.

39. Tesselaar ME, Ouwerkerk J, Nooy MA et al. Risk factors for catheter-related thrombosis in cancer patients. Eur J Cancer 2004; 40: 2253-2259.

40. Lee $A Y$, Levine $M N$, Butler $\mathrm{G}$ et al. Incidence, risk factors, and outcomes of catheter-related thrombosis in adult patients with cancer. J Clin Oncol 2006; 24: 1404-1408.

41. Miyakis S, Lockshin MD, Atsumi T et al. International consensus statement on an update of the classification criteria for definite antiphospholipid syndrome (APS). J Thromb Haemost 2006; 4: 295-306.

42. Zuckerman E, Toubi E, Golan TD et al. Increased thromboembolic incidence in anti-cardiolipin-positive patients with malignancy. Br J Cancer 1995; 72: 447-451. 
CHAPTER 5

43. Pham C, Shen YM. Antiphospholipid antibodies and malignancy. Hematol Oncol Clin North Am 2008; 22: 121-130, vii.

44. Pusterla S, Previtali S, Marziali S et al. Antiphospholipid antibodies in lymphoma: prevalence and clinical significance. Hematol J 2004; 5: 341-346.

45. Yoon KH, Wong A, Shakespeare T, Sivalingam P. High prevalence of antiphospholipid antibodies in Asian cancer patients with thrombosis. Lupus 2003; 12: 112-116.

46. Bezemer ID, van der Meer FJ, Eikenboom JC et al. The value of family history as a risk indicator for venous thrombosis. Arch Intern Med 2009; 169: 610-615. 


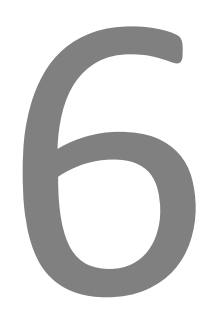

Biomarkers for prediction of central venous catheter related-thrombosis in patients with hematological malignancies

R.S. Boersma, K. Hamulyak, R. van Oerle, A. Tuinenburg, A. J. ten Cate-Hoek, H.C. Schouten

Clin Appl Thromb Hemost. 2015 Apr 17 (epub ahead of print) 


\section{ABSTRACT}

\section{Objective}

In a prospective setting, we aimed to find associations between biomarkers of the hemostatic system and the occurrence of CVC-related thrombosis in patients with hematological malignancies undergoing intensive chemotherapy.

\section{Methods}

The study was conducted between July 2006 and August 2010 at the University Hospital Maastricht, The Netherlands. Consecutive adult patients with hematological malignancies who were going to receive a CVC for intensive chemotherapy were included. The primary endpoints were (a)symptomatic CVC-related thrombosis and CVC-related infections. Blood samples were taken directly after catheterization and easy to determine biomarkers (platelet count, leukocyte count, hemoglobin level) in combination with blood group, FVIII, plasminogen activator inhibitor-1 (PAI-1), activated protein $C(A P C)$ resistance and free Protein $S$ antigen were determined.

\section{Results}

Blood was collected and analyzed from 168 patients. The incidence of symptomatic CVC-related thrombosis was 9\%. In univariate analysis, white blood cell count > $10.6 * 10^{9} / \mathrm{I}$, mean factor VIII activity and PAI-1 > $12.2 \mathrm{IU} / \mathrm{ml}$ were found to be associated with the development of symptomatic CVC-related thrombosis.

\section{Conclusion}

Elevated leukocyte count, high PAI-1 and high factor VIII were associated with an increased incidence of symptomatic CVC-related thrombosis. We hope in future that simple, easy to determine laboratory tests that reflect the hemostatic and fibrinolytic activity in combination with clinical parameters may help to identify hematological patients at highest risk for CVC-related thrombosis and help to tailor the management of thromboprophylaxis in hematological patients undergoing CVC placement. 


\section{INTRODUCTION}

Venous thromboembolism (VTE) occurs commonly in patients with cancer and is the second leading cause of death in these patients. Compared with persons without cancer, the risk of symptomatic VTE in patients with cancer is increased 4- to 7-fold [1, 2]. The occurrence of VTE in cancer patients depends on various patient-, tumor-, and treatment-related risk factors [3]. The incidence of VTE in patients with a hematological malignancy is comparable to the incidence in patients with "high-risk" solid tumors, such as colon, pancreatic and ovarian cancers [4-7].

The guidelines for the treatment and prophylaxis of CVC-related thrombosis recommend against the routine administration of pharmacologic prophylaxis to prevent CVC-related thrombosis [8, 9]. There is no clear consensus regarding the role of either inherited or acquired thrombophilic states in the pathogenesis of CVC-related thrombosis [10], nor is there a clear recommendation on the use of prophylactic measures in this population [9].

The ability to stratify risk would permit appropriate use of VTE prophylaxis, if effective, only in patients at the highest risk. A model-based approach incorporating biomarkers may be the most efficient way to identify cancer patients at highest risk for CVC-related thrombosis and to study prophylaxis strategies in these patients.

It has been shown that risk-scoring models that incorporate clinical and laboratory biomarkers are able to stratify cancer patients into groups at high- or low risk of developing VTE [11-14].

Data about biomarkers for the prediction of VTE in patients with hematological malignancies are scarce because most studies are performed in patients with solid cancer.

A number of laboratory parameters or biomarkers, which are predictive for cancerassociated VTE have been identified. They encompass biomarkers reflecting activation of the blood clotting system such as D-dimer, soluble P-selectin, factor VIII (FVIII) and platelet count, biomarkers reflecting an increase of the inflammatory potential (e.g. Creactive protein (CRP), leukocyte count) and hemoglobin levels and blood group [13, 1517]. Stoffel et al found in patients undergoing intensive cytoreductive treatments for hematological malignancies leukocyte count to be strongly related with the development of thrombotic complications [18]. Del Principe et al demonstrated in a population of patients with acute leukemia a significant correlation between CVCrelated thrombosis and a higher median platelet count [4].

In contrast to a number of studies on the association of biomarkers and cancerassociated deep vein thrombosis or pulmonary embolism, studies investigating the association between biomarkers and symptomatic CVC-related thrombosis in patients with hematological malignancies are very scarce. 
In a prospective setting, we aimed to assess associations between biomarkers of the hemostatic system and the occurrence of CVC-related thrombosis in patients with hematological malignancies. We have chosen well documented, easy to determine and available biomarkers as described in the risk-model of Khorana et al (pre chemotherapy platelet count, leukocyte count, hemoglobin level) $[6,11]$ and combined these with blood group, FVIII, plasminogen activator inhibitor-1 (PAI-1), activated protein C (APC) resistance and free protein $\mathrm{S}$ antigen.

\section{METHODS}

\section{Study design and population}

This study was embedded in a prospective randomized study with the aim to compare the occurrence of CVC-related thrombosis between two locking solutions (Trial registration: NCT 01820962). The study was conducted between July 2006 and August 2010 at the University Hospital Maastricht and the Atrium Medical Center Parkstad Heerlen, The Netherlands. Rationale, study design and selection of the study population have been described in detail previously [19]. The main endpoints of the study were (a)symptomatic thrombosis and/or CVC-related infections.

Consecutive adult patients with hematological malignancies who were going to receive a CVC for intensive chemotherapy, including patients for stem cell transplantation, were included. Exclusion criteria were the presence of a CVC at admission, history of CVC-related thrombosis and/or infection, indication for anticoagulant treatment or prophylaxis, patients with totally implanted catheters and CVCs impregnated with antimicrobial agents. Patients could only be randomized once. The study protocol was approved by the medical ethical committees and all participating patients gave written informed consent.

CVCs were tunneled polyurethane double-lumen catheters (Hickman catheter or Hemoglide; Bard) and were inserted in the right jugular vein by an experienced surgeon in the operating room. Catheters were inserted percutaneously by using the Seldinger technique and most CVCs were placed with the guidance of ultrasound.

The CVC tip was confirmed radiographically to lie in the distal superior vena cava/right atrium. Patients were randomized between locking the catheter lumen with either heparin $5000 \mathrm{IU} / \mathrm{ml}$ (Heparin Leo) or 46.7\% trisodium citrate (Citra-lock; Dirinco) using a volume exactly equivalent to the internal volume noted on each catheter. Since there were no differences in the occurrence of CVC-related thrombosis between the heparin group and the citrate group we have included all patients in the current study regardless of the locking solution used [19]. 
Patients were seen at least daily on the ward and at least weekly on the outpatient department after discharge. In case of a clinical suspicion of a symptomatic CVC-related thrombosis patients were referred to the department of Radiology for Doppler ultrasound. A diagnosis of CVC-related thrombosis was made in case of noncompressibility of the vein, visualization of an echogenic intraluminal mass, and absence of respiratory variation, or monophasic flow [20].

\section{Biomarkers}

Blood samples were taken from all patients directly after catheterization and were collected in K3-EDTA tubes or citrated tubes. Hemoglobin level, platelet count, leukocyte count and blood group were measured immediately after blood collection.

After centrifugation aliquots of plasma samples were frozen at -80 degrees Celcius and stored until analysis. FVIII-activity (ca7000;Siemens), PAI-1 activity (TrinilizePai-1; Stago BNL), APC resistance (Coatest APCresistance; Chromogenix) and free protein S antigen (HemosIL, Instrumention Laboratory), were measured in citrated plasma according to the manufacturers instruction.

\section{Statistical analyses}

After assessment of data distribution patient characteristics and levels of biomarkers were compared between patients with and without symptomatic CVC-related thrombosis using the independent t-test, Mann-Whitney U-test or Chi-square test where appropriate.

Results were tested against an $\alpha$ of 0.05. All analyses were performed using SPSS 20.0 for Windows (SPSS Inc., Chicago, IL, USA).

\section{RESULTS}

Between July 2006 and August 2010248 patients were assessed for eligibility. Thirtyone patients declined to participate and 5 patients could not be randomized due to other reasons. Two hundred and twelve patients receiving a CVC were randomly assigned 1:1 to either locking the CVC with heparin or with citrate. Five patients were excluded ( 2 patients had a solid tumor, 1 patient was lost to follow up, 1 patient eventually did not receive a CVC, 1 patient started systemic anticoagulation). From 39 patients no blood was collected for mainly logistical reasons (CVC placement at night or during weekend). Blood was collected and analyzed from 168 patients in the current study. 
Characteristics of the study population are described in table 1. Overall 9\% (15 out of 168) of patients developed a symptomatic CVC-related thrombosis and one of them developed a pulmonary embolism.

Table 1. Baseline characteristics

\begin{tabular}{|c|c|c|c|}
\hline & $\begin{array}{l}\text { Patients with CVC-related } \\
\text { thrombosis } \\
n=15\end{array}$ & $\begin{array}{l}\text { Patients without CVC- } \\
\text { related thrombosis } \\
n=153\end{array}$ & $P$ \\
\hline Age (years) & $49 \pm 16$ & $56 \pm 11$ & 0.125 \\
\hline Male & $9(60)$ & $96(63)$ & 0.834 \\
\hline $\mathrm{BMI}\left(\mathrm{kg} / \mathrm{m}^{2}\right)$ & $26.2 \pm 5.1$ & $24.5 \pm 4.5$ & 0.178 \\
\hline CVC in situ (days) & $60 \pm 42.2$ & $83 \pm 82.3$ & 0.292 \\
\hline $\begin{array}{l}\text { Time to develop CVC-related } \\
\text { thrombosis (days) }\end{array}$ & $43 \pm 32$ & N/A & \\
\hline Underlying disease & & & Ns \\
\hline AML & $5(33.3)$ & $40(26)$ & \\
\hline ALL & $3(20)$ & $9(5.88)$ & \\
\hline AUL & $0(0)$ & $2(1.3)$ & \\
\hline MM & $2(13.3)$ & 35 (22.9) & \\
\hline Lymphoma & $5(33.3)$ & $47(30.7)$ & \\
\hline Aplastic anemia & $0(0)$ & $3(2)$ & \\
\hline CML & $0(0)$ & $2(1.3)$ & \\
\hline MDS & $0(0)$ & $9(5.88)$ & \\
\hline Amyloidosis & $0(0)$ & $4(2.6)$ & \\
\hline Myelofibrosis & $0(0)$ & $1(0.65)$ & \\
\hline $\mathrm{CLL}$ & $0(0)$ & $1(0.65)$ & \\
\hline Therapy & & & Ns \\
\hline Intensive chemotherapy & $8(53)$ & $60(39)$ & \\
\hline Autologous SCT & $4(27)$ & $70(46)$ & \\
\hline Allogeneic SCT & $3(20)$ & $23(15)$ & \\
\hline$C V C-B S I$ & $6(40)$ & $47(40)$ & 0.460 \\
\hline Lock & & & 0.227 \\
\hline Heparin & 10 & 77 & \\
\hline Sodium & 5 & 76 & \\
\hline
\end{tabular}

Abbreviations: BMI, body mass index; AML, acute myeloid leukemia; ALL, acute lymphoid leukemia; $A U L$, acute undifferentiated leukemia; CML, chronic myeloid leukemia; MDS, myeloid dysplastic syndrome; CLL, chronic lymphoid leukemia; SCT, stem cell transplantation; CVC-BSI, central venous catheter-related bloodstream infection.

Values of continuous variables are expressed as mean \pm standard deviation where appropriate. Categorical numbers are expressed as numbers and percentages. 
Of the biomarkers studied white blood cell count $>10.6^{*} 10^{9} / \mathrm{I}$, mean factor VIII activity and PAl-1 > $12.2 \mathrm{lU} / \mathrm{ml}$ were found to be associated with the development of symptomatic CVCrelated thrombosis in patients with hematological malignancies (table 2).

Factor VIII did not differ according to the type of malignancy (figure 1).

Table 2. Biomarkers and CVC-related thrombosis

\begin{tabular}{|c|c|c|c|}
\hline & $\begin{array}{l}\text { Patients with CVC-related } \\
\text { thrombosis } \\
\mathrm{n}=15\end{array}$ & $\begin{array}{l}\text { Patients without CVC- } \\
\text { related thrombosis } \\
n=153\end{array}$ & $P$ \\
\hline $\operatorname{PLT}\left(* 10^{9} / \mathrm{I}\right)$ & $203(181)$ & $198(145)$ & 0.918 \\
\hline PLT $>350 * 10^{9} / /$ & $5(33)$ & $23(15)$ & 0.074 \\
\hline WBC $\left(* 10^{9} / \mathrm{I}\right)$ & $8.7(2.0-15.5)$ & $6.1(3.7-9.9)$ & 0.449 \\
\hline$W B C>10.6^{*} 10^{9} / I^{*}$ & $7(47)$ & $35(23)$ & 0.042 \\
\hline $\mathrm{Hb}(\mathrm{mmol} / \mathrm{l})$ & $6.3(5.5-7.8)$ & $6.4(5.5-7.6)$ & 0.927 \\
\hline FVIII (\%) & $203(62)$ & $166(59)$ & 0.023 \\
\hline PAI-1 (IU/ml) & $12.2(3.5-22.7)$ & $4.9(1.7-10.2)$ & 0.062 \\
\hline PAl-1 above $12.2 \mathrm{IU} / \mathrm{ml}^{*}$ & $8(53)$ & $34(22)$ & 0.008 \\
\hline Protein S & $100(34)$ & $93(29)$ & 0.417 \\
\hline APC resistance & $3.0(2.63-3.79)$ & $3.11(1.76-4.80)$ & 0.632 \\
\hline Blood Group O & $6(40)$ & $72(47)$ & 0.570 \\
\hline
\end{tabular}

${ }^{*} 75^{\text {th }}$ percentile

Abbreviations: PLT, platelets; WBC, white blood cell count; $\mathrm{Hb}$, hemoglobin; PAl plasminogen activator inhibitor; APC, activated protein $\mathrm{C}$.

Values of continuous variables are expressed as mean \pm standard deviation or median and interquartile range where appropriate. Categorical numbers are expressed as numbers and percentages. 


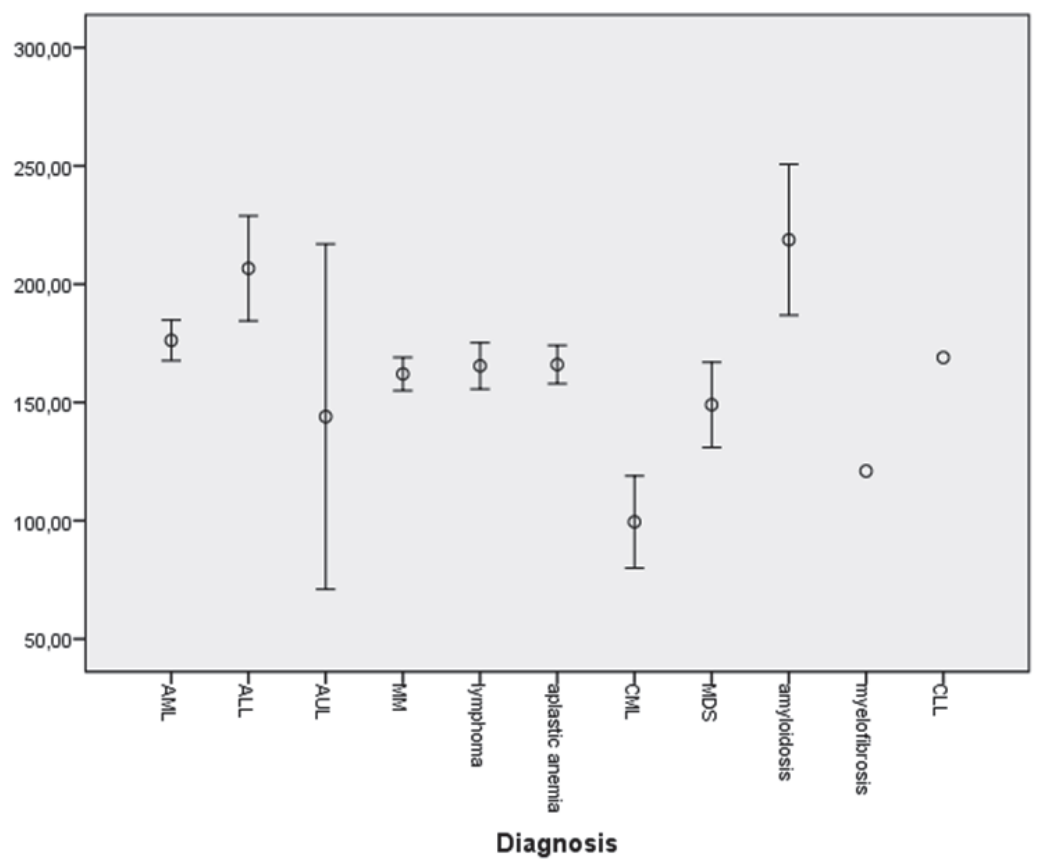

Figure 1. Distribution of factor VIII levels according to the type of hematological malignancy Factor VIII levels did not differ statistically significant $(p=0.214)$

The mean platelet level at the moment of inserting the CVC did not differ between patients with and without a CVC-related thrombosis. When we limited our analysis to patients with acute leukemia we found the mean platelet level at the moment of inserting the CVC to be $178 * 10^{9} /$ (SD 204) in the patients with acute leukemia developing a CVC-related thrombosis compared to $89 * 10^{9} /$ I (SD 89) in the patients with acute leukemia not developing a CVC-related thrombosis $(P=0.019)$.

\section{DISCUSSION}

This is the first prospective study evaluating associations between laboratory biomarkers and the occurrence of symptomatic CVC-related thrombosis in patients with hematological malignancies undergoing intensive chemotherapy. The observed incidence of symptomatic CVC-related thrombosis in our study is $9 \%$ which is clinically relevant and consistent to incidences reported in other studies [21].

In univariate analysis, white blood cell count $>10.6 * 10^{9} /$, mean factor VIII activity and PAI-1 > $12.2 \mathrm{IU} / \mathrm{ml}$ were found to be associated with the development of symptomatic CVC-related thrombosis in patients with hematological malignancies. 
PAI-1 inactivates tissue-type plasminogen activator (tPA) and urokinase-type plasminogen activator (UPA). As a consequence of elevated PAI-1 levels, less plasminogen is converted into plasmin thereby reducing fibrinolysis. This process favors tumor cell invasion and metastasis [22]. Reduced plasma fibrinolytic activity due to elevated plasma levels of PAI-1 has been observed in various severity of arterial thrombosis [23-25]. The role of PAI-1 in venous thrombosis remains controversial. Although the authors of several studies have found a positive relation with venous thrombosis, others have not [26-28]. In our study population $53 \%$ of patients with a CVC-related thrombosis had a PAI-1 above the $75^{\text {th }}$ percentile of the study population compared to only $22 \%$ in the patients without a CVC-related thrombosis $(P=0.008)$. We have not tested the frequencies of heterozygous and homozygous PAI-1 $4 \mathrm{G}$ polymorphisms. To the best of our knowledge no other studies have been published describing the relationship between PAI-1 and CVC-related thrombosis in hematological patients.

Elevated white blood cell count has been established as an independent risk factor for venous thromboembolic events in patients undergoing stem cell transplantation or intensive chemotherapy [18].

In our study population we established a baseline white blood cell count above the $75^{\text {th }}$ percentile of the study population to be a risk factor for CVC-related thrombosis.

Whether leukocytes themselves are causative or rather act as a surrogate marker for an underlying inflammatory process has not been conclusively established. A pathogenic mechanism is not definitely elucidated. Neutrophil granulocytes have been implicated to play a central role as substrate for platelets. The latter can use adhesion molecules on their membranes and find ligands on the neutrophil surface causing mutual activation and procoagulant conditions [29].

In addition, we detected high levels of FVIII in our population of patients with hematological malignancies (figure 1), probably reflecting the acute phase reactive nature of this procoagulant factor. Here also, our data are in accordance with the few available studies that reported increased FVIII levels in patients with solid tumors and hematological malignancies [17, 30-32]. High FVIII levels constitute a prevalent, dosedependent risk factor for VTE [17]. We demonstrated that patients with a hematological malignancy with a CVC-related thrombosis have a significantly higher FVIII level compared to patients without a CVC-related thrombosis. In the group without a CVCrelated thrombosis one patient had a factor VIII level of $8 \%$. When this patient was excluded from the analysis the difference between the two groups remained significantly different.

Reports about the thrombosis risk according to FVIII in cancer patients are rare. Vormittag et al showed in 2009 that a high FVIII plasma level is a significant risk factor for symptomatic VTE in cancer patients [17]. Van Marion et al showed in a prospective study in 135 multiple myeloma patients and 124 age- and sex-matched healthy controls higher baseline FVIII levels in patients compared to controls, but was not able to 
demonstrate an increased thrombosis risk [33]. Van Rooden et al found patients with the highest levels of FVIII to be at a not statistically significant higher risk of asymptomatic CVC-related thrombosis [31].

In general we could not demonstrate a role for high platelet count in developing CVCrelated thrombosis in our population. Only when we limited our analysis to patients with acute leukemia we found a significant higher mean platelet count in the patients with CVC-related thrombosis. Del Principe et al also demonstrated in a population with patients with acute leukemia a significant correlation between CVC-related thrombosis and a higher median platelet count [4]. A possible explanation of this relationship in acute leukemia might be the severe hyper-coagulable state due to the expression of tissue factor by the leukemic blasts or the release of pro-coagulant factors from the blast granular fraction [4].

Thrombocytosis is not uncommon in cancer patients and evidence exists that platelets play an important role in tumor angiogenesis [34].

Mandala et al showed a strong association between elevated basal platelet counts and the risk for VTE during adjuvant chemotherapy [35]. Simanek et al demonstrated a high platelet count to be strongly associated with the occurrence of VTE in cancer patients with active disease, exceeding an at least 3-fold increased risk at a cut off at the $95^{\text {th }}$ percentile of their patient cohort $\left(443 * 10^{9} / 1\right)[16]$. These results are in agreement with the retrospective study of Zakai and the prospective study of Khorana that used a cut off of $350 * 10^{9} / /$ [6]. Only a few patients with hematological malignancies were included in these analyses.

The reason why platelet count in our study was not a strong risk factor as reported in other malignancies may be the relatively low platelet count patients with a hematological malignancy often present with.

Some limitations of this study have to be considered. Our study had a limited sample size and therefore only a limited number of events. Only univariate analyses were performed. The biomarkers were measured only once at study inclusion. We cannot exclude that biomarker levels might have changed during the course of disease and under treatment.

In conclusion, CVC-related thrombosis in patients with a hematological malignancy is prevalent. Simple, easy to determine laboratory tests such as white blood cell count, factor VIII and PAI-1 in combination with clinical parameters may help to identify hematological patients at highest risk for CVC-related thrombosis and help to tailor the management of thromboprophylaxis 


\section{REFERENCES}

1. Blom JW, Doggen CJ, Osanto S, Rosendaal FR. Malignancies, prothrombotic mutations, and the risk of venous thrombosis. Jama 2005; 293: 715-722.

2. Heit JA, Silverstein MD, Mohr DN et al. Risk factors for deep vein thrombosis and pulmonary embolism: a population-based case-control study. Arch Intern Med 2000; 160: 809-815.

3. Prandoni P, Falanga A, Piccioli A. Cancer and venous thromboembolism. Lancet Oncol 2005; 6: 401-410.

4. Del Principe MI, Buccisano F, Maurillo $L$ et al. Infections increase the risk of central venous catheterrelated thrombosis in adult acute myeloid leukemia. Thromb Res 2013; 132: 511-514.

5. Mohren M, Markmann I, Jentsch-Ullrich $\mathrm{K}$ et al. Increased risk of venous thromboembolism in patients with acute leukaemia. Br J Cancer 2006; 94: 200-202.

6. Khorana AA, Francis CW, Culakova E, Lyman GH. Risk factors for chemotherapy-associated venous thromboembolism in a prospective observational study. Cancer 2005; 104: 2822-2829.

7. Khorana AA, Francis CW, Culakova E et al. Thromboembolism in hospitalized neutropenic cancer patients. J Clin Oncol 2006; 24: 484-490.

8. Zwicker J, Connolly G, Carrier M et al. Catheter-associated deep vein thrombosis of the upper extremity in cancer patients: guidance from the SSC of the ISTH. J Thromb Haemost 2014; 12(5): 796-800.

9. Schiffer CA, Mangu PB, Wade JC et al. Central venous catheter care for the patient with cancer: American Society of Clinical Oncology clinical practice guideline. J Clin Oncol 2013; 31: 1357-1370.

10. Boersma RS, Hamulyak K, Cate HT, Schouten HC. Congenital thrombophilia and central venous catheterrelated thrombosis in patients with cancer. Clin Appl Thromb Hemost 2010; 16: 643-649.

11. Khorana AA, Kuderer NM, Culakova E et al. Development and validation of a predictive model for chemotherapy-associated thrombosis. Blood 2008; 111: 4902-4907.

12. Ay C, Dunkler D, Marosi C et al. Prediction of venous thromboembolism in cancer patients. Blood 2010; 116: 5377-5382.

13. Pabinger I, Thaler J, Ay C. Biomarkers for prediction of venous thromboembolism in cancer. Blood 2013; 122: 2011-2018.

14. Ay C, Vormittag R, Dunkler D et al. D-dimer and prothrombin fragment $1+2$ predict venous thromboembolism in patients with cancer: results from the Vienna Cancer and Thrombosis Study. J Clin Oncol 2009; 27: 4124-4129.

15. Ay C, Pabinger I. Predictive potential of haemostatic biomarkers for venous thromboembolism in cancer patients. Thromb Res 2012; 129 Suppl 1: S6-9.

16. Simanek R, Vormittag R, Ay $C$ et al. High platelet count associated with venous thromboembolism in cancer patients: results from the Vienna Cancer and Thrombosis Study (CATS). J Thromb Haemost 2010; 8: $114-120$.

17. Vormittag R, Simanek R, Ay C et al. High factor VIII levels independently predict venous thromboembolism in cancer patients: the cancer and thrombosis study. Arterioscler Thromb Vasc Biol 2009; 29: 2176-2181.

18. Stoffel N, Rysler C, Buser A et al. Leukocyte count and risk of thrombosis in patients undergoing haematopoietic stem cell transplantation or intensive chemotherapy. Thromb Haemost 2010; 103: 12281232.

19. Boersma RS, Jie KS, Voogd AC et al. Concentrated citrate locking in order to reduce the long-term complications of central venous catheters: a randomized controlled trial in patients with hematological malignancies. Support Care Cancer 2015; 23 (1): 37-45.

20. Baxter GM, Kincaid W, Jeffrey RF et al. Comparison of colour Doppler ultrasound with venography in the diagnosis of axillary and subclavian vein thrombosis. Br J Radiol 1991; 64: 777-781.

21. Boersma RS, Jie KS, Verbon A et al. Thrombotic and infectious complications of central venous catheters in patients with hematological malignancies. Ann Oncol 2008; 19: 433-442.

22. Andreasen PA, Kjoller L, Christensen L, Duffy MJ. The urokinase-type plasminogen activator system in cancer metastasis: a review. Int J Cancer 1997; 72: 1-22. 


\section{CHAPTER 6}

23. Yucel O, Karahan O, Zorlu A, Manduz S. Familial genetic risk factors in premature cardiovascular disease: a family study. Mol Biol Rep 2012; 39: 6141-6147.

24. Lane DA, Grant PJ. Role of hemostatic gene polymorphisms in venous and arterial thrombotic disease. Blood 2000; 95: 1517-1532.

25. Wang J, Wang $\mathrm{C}$, Chen $\mathrm{N}$ et al. Association between the plasminogen activator inhibitor-1 4G/5G polymorphism and risk of venous thromboembolism: a meta-analysis. Thromb Res 2014; 134: 1241-1248.

26. Arslan S, Manduz S, Epozturk K et al. Association of deep venous thrombosis with prothrombotic gene polymorphism identified in lung cancer cases. Mol Biol Rep 2011; 38: 2395-2400.

27. Folsom AR, Cushman M, Heckbert SR et al. Prospective study of fibrinolytic markers and venous thromboembolism. J Clin Epidemiol 2003; 56: 598-603.

28. Meltzer ME, Lisman T, de Groot PG et al. Venous thrombosis risk associated with plasma hypofibrinolysis is explained by elevated plasma levels of TAFI and PAI-1. Blood 2010; 116: 113-121.

29. Falanga A, Marchetti M, Vignoli A et al. Leukocyte-platelet interaction in patients with essential thrombocythemia and polycythemia vera. Exp Hematol 2005; 33: 523-530.

30. Auwerda JJ, Sonneveld P, de Maat MP, Leebeek FW. Prothrombotic coagulation abnormalities in patients with newly diagnosed multiple myeloma. Haematologica 2007; 92: 279-280.

31. Van Rooden CJ, Rosendaal FR, Meinders AE et al. The contribution of factor $V$ Leiden and prothrombin G20210A mutation to the risk of central venous catheter-related thrombosis. Haematologica 2004; 89: 201-206.

32. Ratcliffe $M$, Broadfoot $C$, Davidson $M$ et al. Thrombosis, markers of thrombotic risk, indwelling central venous catheters and antithrombotic prophylaxis using low-dose warfarin in subjects with malignant disease. Clin Lab Haematol 1999; 21: 353-357.

33. van Marion AM, Auwerda JJ, Lisman T et al. Prospective evaluation of coagulopathy in multiple myeloma patients before, during and after various chemotherapeutic regimens. Leuk Res 2008; 32: 1078-1084.

34. Sierko E, Wojtukiewicz MZ. Platelets and angiogenesis in malignancy. Semin Thromb Hemost 2004; 30: 95-108

35. Mandala M, Barni S, Prins $M$ et al. Acquired and inherited risk factors for developing venous thromboembolism in cancer patients receiving adjuvant chemotherapy: a prospective trial. Ann Oncol 2010; 21: 871-876. 


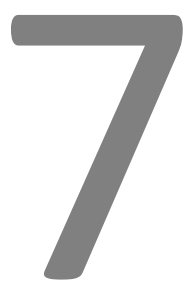

General conclusions and further perspectives

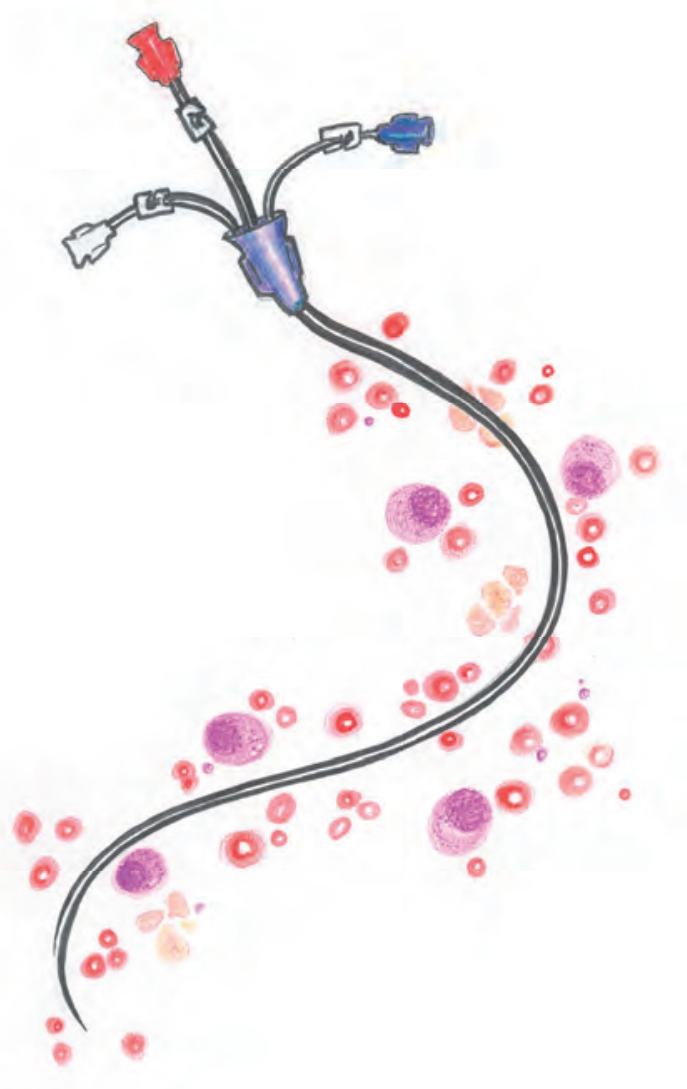



This thesis addresses several major topics regarding long term central venous catheter (CVC)-related complications in patients with hematological malignancies undergoing intensive chemotherapy. Firstly, a summary is given about what is known concerning long term complications of CVCs in hematological patients and local practices concerning CVCs in hematological patients are described. Secondly, whether concentrated citrate can reduce the incidence of CVC-related infections and thrombosis in hematological patients as it does in hemodialysis patients. Thirdly, whether biomarkers can predict the risk of CVC-related thrombosis in hematological patients undergoing intensive chemotherapy.

\section{CVC-RELATED INFECTIONS}

Reviewing the literature concerning CVC-related blood stream infections (BSI) in hematological patients undergoing intensive chemotherapy we find an incidence that varies between $0.0-40.6 \%$ which makes it a clinically relevant problem [1]. In our randomized controlled study we find an incidence of CVC-related bloodstream infections of $33 \%$. In $46 \%$ of CVC-BSI cultures yielded gram-positive micro-organisms, predominantly coagulase-negative staphylococci (CNS) and Staphylococcus hemolyticus.

An incidence of $60 \%$ of CVC-BSI was seen in patients treated with intensive chemotherapy compared to only $7 \%$ of CVC-BSI in patients undergoing an autologous stem cell transplantation. An underlying diagnosis of acute leukemia is significantly associated with an elevated risk of CVC-related BSI in more studies [2-5]. This difference is probably explained by differences in the duration of insertion of the CVC, duration of neutropenia and mucotoxic effects of the chemotherapy. This raises the question whether patients with an acute leukemia undergoing intensive chemotherapy need other CVCs, e.g. impregnated with antimicrobial agents.

Diagnosing CVC-related bloodstream infections in hematological patients is notoriously difficult. The clinical presentation of CVC-related infections consists of nonspecific systemic manifestations and local manifestations at the CVC insertion site and may be subtle or absent in neutropenic cancer patients [6]. The most common techniques are based on removal of the CVC. Since these CVCs are often integral to patient care in hematological patients, CVCs are mostly not removed and antibiotics are started. CVC-related infections mostly involves coagulase-negative staphylococci that originate from the resident commensal flora of skin and gut and can occur through either an exogenous route (via the catheter hub) or an endogenous route as a result of disrupted mucosal barriers of the oral cavity and gastrointestinal tract [7]. Hematological patients undergoing intensive chemotherapy often experience mucositis which leads to translocation of gram positive and gram negative micro- organisms which makes that preventive strategies should not only be focused on the skin [8]. The CVC device itself is usually presumed to be the source of bacteremia, whereas the 
mucosal barrier may well be the origin [7]. They are nonetheless classified as CVCrelated infections. The $\mathrm{CDC}$ (Centers for disease control and prevention) recently even developed a new definition termed 'mucosal barrier injury-laboratory-confirmed bloodstream infection' (MBI-LCBI). It applies to bacteremic patients (with Viridans group streptococci, Bacteroides spp., Candida spp., Clostridium spp., Enterococcus spp., Fusobacterium spp., Peptostreptoccus spp., Prevotella spp., Veillonella spp., or Enterobacteriaceae) with central venous catheters with mucosal injury related to post chemotherapy neutropenia or graft-versus-host disease [6]. Metzger et al evaluated the impact of this new definition on their hematological population. Most CVC-related infections they identified in their population met the MBI-LCBI criteria. Prevention efforts did not reduce these infections but did reduce CVC-related infections [9].

Applying these new criteria to our patient population with probable and definite CVC-related infections about 50\% met the MBI-LCBI criteria.

\section{CVC-RELATED THROMBOSIS}

The cumulative incidence of symptomatic CVC-related thrombosis was $10 \%$ in the heparin group and 5\% in the TSC group which is consistent to the incidences found in other studies [1]. Loss of catheter function is probably the most frustrating complication since a CVC in hematological patients is often a life line. Specific attention to the preservation of the CVC should be made. CVC-related thrombosis can delay the administration of therapy and expose patients to the hazards of 3 months therapeutic anticoagulation and gives a higher risk of CVC-related infections.

The guidelines for the treatment and prophylaxis of CVC-related thrombosis recommend against the routine administration of pharmacologic prophylaxis to prevent CVC-related thrombosis $[10,11]$.

In this thesis elevated leukocyte count, factor VIII and plasminogen activator inhibitor-1 were found to be potential interesting biomarkers for the development of symptomatic CVC-related thrombosis. Further investigation determining baseline clinical and laboratory parameters to help identify hematological patients at highest risk for CVC-related thrombosis and infections is needed. This will help to tailor the management of central venous catheters in hematological patients undergoing intensive chemotherapy.

\section{PREVENTION OF LONG-TERM COMPLICATIONS OF CVCS}

Prevention of long term complications is of utmost importance. The paradigm for dealing with these long-term complications needs to shift from treatment to prevention. There is accumulating evidence showing that CVC-related thrombosis and 
infections are interrelated and can therefore not be seen as separate entities. There seems to be a bi-directional relationship [12-14]. A major contributing factor in both CVC-related thrombosis and CVC-related infections might be fibrin sheath formation around the external portion of the catheter and within the catheter lumen [15]. Microbial pathogens easily adhere to these thrombin sheaths. Interventions designed to decrease fibrin deposition and thrombus formation have the potential to reduce CVCrelated infections and thrombosis. Abdelkefi et al showed a significant reduction in CVCrelated thrombosis and infections using a continuous infusion of unfractionated heparin at 100 units $/ \mathrm{kg} /$ day in 108 adults and children undergoing bone marrow transplantation [16]. Van Rooden et al showed three times weekly urokinase rinsing to reduce the incidence of CVC-related infections with coagulase negative staphylococcus [17].

The use of trisodium citrate (TSC) lowered the incidence of CVC-related bloodstream infections (BSI) in hemodialysis patients from 4.1 CVC-related BSI to 1.1 per 1000 catheter days. In our hematological patient population we found no differences in the incidence of CVC-related bloodstream infections with heparin locking and TSC locking. Unexpectedly we found seven times more CVC-BSI with gram negative organisms in the heparin group than in the TSC group. The reason we did not see statistically significant differences on BSI with CNS is because alternative sources of bacteremia like microbial translocation during chemotherapy-induced mucositis have been important as well in our patient population, the severe immunocompromised character of our patient population and the intensive use of our CVCs. In hematological patients CVCs are used very intensively for administering chemotherapy, parenteral nutrition, blood products as well for taking blood samples several times a day. Hematologist have no choice than to "mistreat" CVCs.

The institution of a bundle of aseptic techniques and the implementation of surveillance with feedback and following training [5] decreases the risk of CVC-related infections [18]. Since hematological patients often experience mucositis which leads to translocation of gram positive and gram negative micro-organisms preventive strategies should not only be focused on these aseptic techniques and the skin [8]. Other interventions like antimicrobial impregnation or coating of the CVC or different locking solutions may be necessary to further reduce the risk of CVC-related infections in this specific population.

\section{FUTURE PERSPECTIVES}

CVC-related infections and thrombosis should not be considered as a result of modern care but must be one of the priority targets of a multidisciplinary approach emphasizing quality-of-care improvement. The paradigm for dealing with these long-term complications needs to further shift from treatment to prevention. The target should be a multidisciplinary approach involving hematologists, nurses, interventional radiologists, 
surgeons, anesthesiologists and infectious disease specialists to decrease the risk of CVC-related thrombosis and infections to zero. In every hospital should be a dedicated CVC team using a CVC care bundle and international standardized guidelines concerning the insertion and management of the CVC. Hematological centers should monitor CVCrelated infections and report them in an uniform methodology. A national registry for CVC-related infections in hematological patients should be initiated. The application of a valid, standardized surveillance definition specifically for hematological patients separating $\mathrm{MBI}-\mathrm{LCBI}$ from non-MBI-LCBI is then imperative.

The ideal device for hematological patients should be easily and safely placed and removed, should facilitate easy blood withdrawal and drug delivery, exhibit a low complication rate and be well tolerated by the patient.

Hematological patients undergoing intensive chemotherapy with a high risk of CVCrelated BSI should probably get a kind of CVC with a different surface coating with antibiotics, antiseptics or silver nanoparticles or a different locking solution.

PICCS (peripherally inserted central venous catheters) might be a safe alternative for CVCs. PICC use in hematological patients is associated with the same or a lower rate of CVC-BSI relative to that associated with CVCs [19-21]. Older reports describe high rates of CVC-related thrombosis in patients with PICCS. More recent reports describe much lower rates of CVC-related thrombosis [19-21]. This is probably the result of the use of ultrasound to ensure correct catheter placement and improved biomaterials. The use of PICCS in hematological patients undergoing intensive chemotherapy has been increasing the last years.

There is need for a better understanding of the mechanism of catheter thrombosis so that more targeted therapy can be developed. Yau et al suggest that CVC-related thrombosis is triggered via the contact pathway by activating factor XII and that the extrinsic pathway plays little or no role in this process [22-24]. They studied the effect of antisense oligonucleotides-induced depletion of factor VII, FXI, FXII or high-molecularweight kininogen (HK) on thrombus formation induced by polyurethane catheters inserted into rabbit jugular veins. FXII or FXI reduction prolonged time to thrombus formation more than two-fold, while reducing factor VII or HK had little effect providing evidence that CVC-related thrombosis is triggered via the contact pathway and indentifying factor XII and factor XI as potential targets to attenuate CVC-related thrombosis [22].

We need baseline clinical and laboratory parameters to help identify hematological patients at highest risk for CVC-related thrombosis which may help us to tailor the management of thromboprophylaxis in hematological patients undergoing intensive chemotherapy. The specificity of biomarkers would be considerably improved by combining clinical factors with biomarkers and creating risk assessment models as Khorana did for chemotherapy-associated thrombosis in ambulatory cancer patients [25]. Khorana incorporated site of cancer, platelet count, hemoglobin and/or use of erythropoiesis-stimulating agents, leukocyte count, and body mass index. Ay et al 
expanded this model with soluble P-selectin and D-dimer in a heterogeneous cohort of cancer patients undergoing surgery, radiotherapy and even untreated cancer patients [26].

The measurement of thrombin generation (TG) might represent a new method for global assessment of the hypercoagulable state in cancer patients and could help identify cancer patients at increased risk of VTE. However, thrombin generation assays as new global coagulation tests have not been introduced into routine clinical practice so far due to the lack of standardization of preanalytical and analytical conditions, which are responsible for the large inter-laboratory variations of test results, which are currently observed [27]. Future prospective and interventional studies are needed to elucidate the clinical utility of thrombin generation assays in hematological patients with specific attention to the preanalytical conditions.

In conclusion, central venous catheters in hematological patients are 'a life line'. In case of CVC-related complications they can be 'risky lifelines' and a 'pain in the neck'. These long term complications impair CVC functioning, result in morbidity and pose hematologists with difficult questions on what anticoagulant treatment to choose in these often thrombocytopenic patients and whether this 'life line' must be removed. The paradigm for dealing with these long-term complications needs to further shift from treatment to prevention with specific attention for this specific group of patients. 


\section{REFERENCES}

1. Boersma RS, Jie KS, Verbon A et al. Thrombotic and infectious complications of central venous catheters in patients with hematological malignancies. Ann Oncol 2008; 19: 433-442.

2. Worth LJ, Seymour JF, Slavin MA. Infective and thrombotic complications of central venous catheters in patients with hematological malignancy: prospective evaluation of nontunneled devices. Support Care Cancer 2009; 17: 811-818.

3. Nosari AM, Nador G, De Gasperi A et al. Prospective monocentric study of non-tunnelled central venous catheter-related complications in hematological patients. Leuk Lymphoma 2008; 49: 2148-2155.

4. Digiorgio MJ, Fatica C, Oden M et al. Development of a modified surveillance definition of central lineassociated bloodstream infections for patients with hematologic malignancies. Infect Control Hosp Epidemiol 2012; 33: 865-868.

5. Chaberny IF, Ruseva E, Sohr D et al. Surveillance with successful reduction of central line-associated bloodstream infections among neutropenic patients with hematologic or oncologic malignancies. Ann Hematol 2009; 88: 907-912.

6. Raad I, Chaftari AM. Advances in prevention and management of central line-associated bloodstream infections in patients with cancer. Clin Infect Dis 2014; 59 Suppl 5: S340-343.

7. Richters $A$, van Vliet $M$, Peer PG et al. Incidence of and risk factors for persistent gram-positive bacteraemia and catheter-related thrombosis in haematopoietic stem cell transplantation. Bone Marrow Transplant 2014; 49: 264-269.

8. Costa SF, Miceli MH, Anaissie EJ. Mucosa or skin as source of coagulase-negative staphylococcal bacteraemia? Lancet Infect Dis 2004; 4: 278-286.

9. Metzger KE, Rucker $\mathrm{Y}$, Callaghan $\mathrm{M}$ et al. The burden of mucosal barrier injury laboratory-confirmed bloodstream infection among hematology, oncology, and stem cell transplant patients. Infect Control Hosp Epidemiol 2015; 36: 119-124.

10. Zwicker J, Connolly G, Carrier M et al. Catheter-associated deep vein thrombosis of the upper extremity in cancer patients: guidance from the SSC of the ISTH. J Thromb Haemost 2014; 12: 796-800

11. Schiffer CA, Mangu PB, Wade JC et al. Central venous catheter care for the patient with cancer: American Society of Clinical Oncology clinical practice guideline. J Clin Oncol 2013; 31: 1357-1370.

12. van Rooden CJ, Schippers EF, Barge RM et al. Infectious complications of central venous catheters increase the risk of catheter-related thrombosis in hematology patients: a prospective study. J Clin Oncol 2005; 23: 2655-2660.

13. Lordick F, Hentrich M, Decker T et al. Ultrasound screening for internal jugular vein thrombosis aids the detection of central venous catheter-related infections in patients with haemato-oncological diseases: a prospective observational study. Br J Haematol 2003; 120: 1073-1078.

14. Timsit JF, Farkas JC, Boyer JM et al. Central vein catheter-related thrombosis in intensive care patients: incidence, risks factors, and relationship with catheter-related sepsis. Chest 1998; 114: 207-213.

15. Mehall JR, Saltzman DA, Jackson RJ, Smith SD. Fibrin sheath enhances central venous catheter infection. Crit Care Med 2002; 30: 908-912.

16. Abdelkefi A, Torjman L, Ladeb S et al. Randomized trial of prevention of catheter-related bloodstream infection by continuous infusion of low-dose unfractionated heparin in patients with hematologic and oncologic disease. J Clin Oncol 2005; 23: 7864-7870.

17. van Rooden CJ, Schippers EF, Guiot HF et al. Prevention of coagulase-negative staphylococcal central venous catheter-related infection using urokinase rinses: a randomized double-blind controlled trial in patients with hematologic malignancies. J Clin Oncol 2008; 26: 428-433.

18. Pronovost $P$, Needham D, Berenholtz $S$ et al. An intervention to decrease catheter-related bloodstream infections in the ICU. N Engl J Med 2006; 355: 2725-2732.

19. Bellesi S, Chiusolo P, De Pascale G et al. Peripherally inserted central catheters (PICCS) in the management of oncohematological patients submitted to autologous stem cell transplantation. Support Care Cancer 2013; 21: 531-535. 
20. Sakai T, Kohda K, Konuma $Y$ et al. A role for peripherally inserted central venous catheters in the prevention of catheter-related blood stream infections in patients with hematological malignancies. Int J Hematol 2014; 100: 592-598.

21. Lim MY, Al-Kali A, Ashrani AA et al. Comparison of complication rates of Hickman((R)) catheters versus peripherally inserted central catheters in patients with acute myeloid leukemia undergoing induction chemotherapy. Leuk Lymphoma 2013; 54: 1263-1267.

22. Yau JW, Liao P, Fredenburgh JC et al. Selective depletion of factor XI or factor XII with antisense oligonucleotides attenuates catheter thrombosis in rabbits. Blood 2014; 123: 2102-2107.

23. Yau JW, Stafford AR, Liao P et al. Corn trypsin inhibitor coating attenuates the prothrombotic properties of catheters in vitro and in vivo. Acta Biomater 2012; 8: 4092-4100.

24. Yau JW, Stafford AR, Liao $P$ et al. Mechanism of catheter thrombosis: comparison of the antithrombotic activities of fondaparinux, enoxaparin, and heparin in vitro and in vivo. Blood 2011; 118: 6667-6674.

25. Khorana AA, Kuderer NM, Culakova $E$ et al. Development and validation of a predictive model for chemotherapy-associated thrombosis. Blood 2008; 111: 4902-4907.

26. Ay C, Dunkler D, Marosi C et al. Prediction of venous thromboembolism in cancer patients. Blood 2010; 116: 5377-5382.

27. Pabinger I, Ay C. Biomarkers and venous thromboembolism. Arterioscler Thromb Vasc Biol 2009; 29: 332336. 

Summary

Nederlandse samenvatting

Dankwoord

Curriculum Vitae

List of publications

Valorization

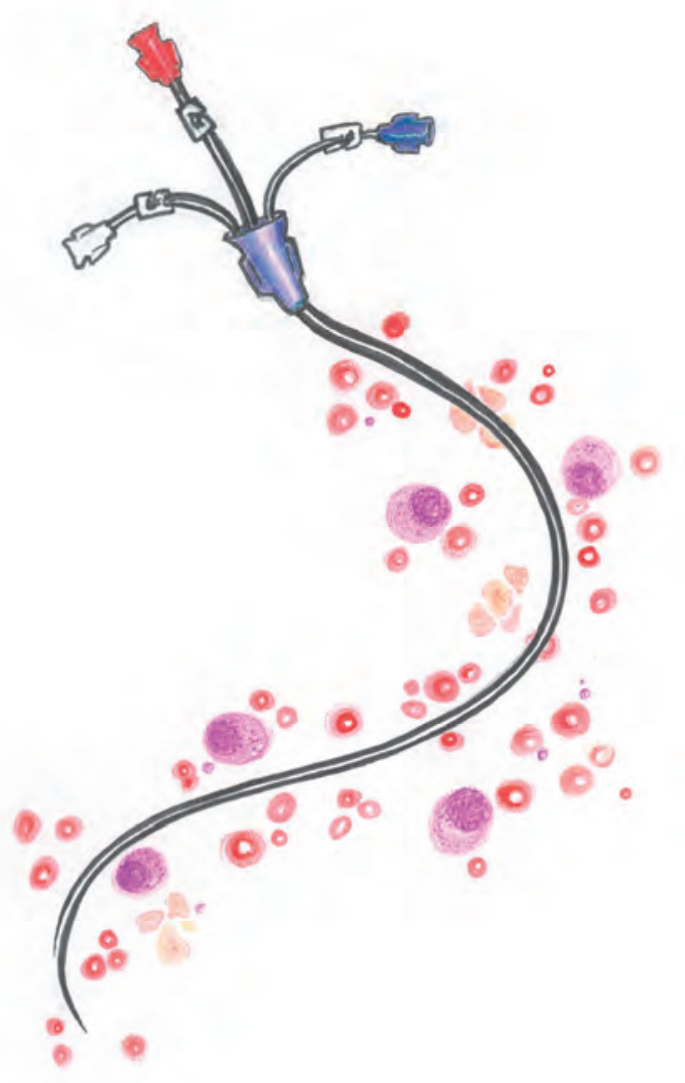





\section{SUMMARY}

This thesis concerns long term central venous catheter (CVC)-related complications in patients with hematological malignancies undergoing intensive chemotherapy. CVCs are frequently used in patients with hematological malignancies undergoing intensive chemotherapy in order to administer chemotherapy, stem cell infusions, blood products, medication, parenteral hyperalimentation as well as for blood sampling. Reported complications consist of mechanical complications during or directly after the insertion and long-term complications such as infections and thrombosis [1]. CVCrelated infections and thrombosis result in patient morbidity, significant increases in the length of hospitalization and medical care costs [2]. Many studies have addressed the incidence and associated risk factors of CVC-related infections and thrombosis in patients with solid tumors, but only very limited data are available on hematological patients undergoing intensive chemotherapy.

A general introduction and outline of the thesis is presented in chapter 1 .

In chapter 2, we present a systematic review of the studies published on long term CVC-related complications in hematological patients undergoing intensive chemotherapy. The incidence, pathogenesis, risk factors, diagnosis, treatment and preventive measures are described. The incidence of a symptomatic CVC-related thrombosis was reported to vary between 1.2-18.0\%. Pulmonary embolism occurs in 15-25\% of patients with a symptomatic CVC-related thrombosis, postphlebitic syndrome arises in $14.8 \%$ of patients with an upper extremity deep vein thrombosis and there is an increased risk of CVC-related infections. The guidelines for the treatment and prophylaxis of CVC-related thrombosis recommend against the routine administration of pharmacologic prophylaxis to prevent CVC-related thrombosis [3, 4].

The incidence of a CVC-related bloodstream infection varies between $0.0-40.6 \%$. CVC-related infections in hematological patients are usually caused by coagulasenegative staphylococcus (62.5\%), Staphylococcus aureus (4.2\%), Gram negative bacilli such as Enterobacteriacae, Escherichia coli and Pseudomonas spp (29\%) or Candida spp (4.2\%) [5]. Diagnosis of CVC-related infections is notoriously difficult. There is need for a specific approach regarding diagnosis and treatment of CVC-related infections and thrombosis with specific attention for preservation of the CVC.

Since CVC-related thrombosis and infections are interrelated [6, 7], interventions designed to decrease fibrin deposition and thrombus formation have the potential to reduce $\mathrm{CVC}$-related infections and trombosis.

The results of a survey among 23 Dutch and Belgian hematological centres applying intensive chemotherapy according to the HOVON (Hemato Oncology Foundation for adults in the Netherlands) protocols are outlined in chapter 3 . The survey contained 30 questions regarding local policies and protocols of catheter type, catheter insertion, catheter care, diagnosis, prevention and treatment of CVC-related thrombosis and 
infections. The survey was sent to an experienced hematologist in every centre. The local protocols and policies differ greatly among the centres probably reflecting wide differentiations in practice across Europe. It also shows lack of evidence concerning CVC-related thrombosis and infections which may cause morbidity in hematological patients.

Fibrin sheath formation around the external portion of the CVC and within the catheter lumen has been implicated as a major contributing factor in both occlusive and infectious events [8]. Trisodium citrate (TSC) prevents CVC-related thrombosis via local anticoagulation by chelating ionized calcium. TSC exerts a broad antimicrobial and antiyeast effect [9]. TSC lowered the incidence of CVC-related bloodstream infections in hemodialysis patients from 4.1 CVC-related BSI per 1000 catheters days to 1.1 per 1000 catheter days [10]. Therefore we performed a randomized controlled trial to determine the efficacy of TSC as a locking solution compared to heparin in preventing CVC-related infections and thrombosis in hematological patients undergoing intensive chemotherapy. The results are described in chapter 4. Thirty-four episodes of CVCrelated bloodstream infections were found in the 108 patients who where randomized to locking with heparin compared with 35 episodes in the 99 patients who where randomized to locking with TSC $(p=0.654)$. We did find seven times more CVC-BSI with gram negative rods in CVCs locked with heparin $(p=0.041)$. The cumulative incidence of symptomatic thrombosis was $10 \%$ in the heparin group and $5 \%$ in the TSC group (hazard ratio 0.525 ; 95\% confidence interval 0.182-1.512).

An incidence of $60 \%$ of CVC-BSI was seen in patients treated with intensive chemotherapy compared to only $7 \%$ of CVC-BSI in patients undergoing an autologous stem cell transplantation.

In chapter 5 we present a narrative review which discusses the main studies that examined whether a state of thrombophilia increases the risk of CVC-related thrombosis in patients with cancer. An extensive literature review of the studies published between 1990 and January 2010 was performed using the MEDLINE database. The prevalence of factor $V$ Leiden in cancer patients ranges from $4.0-9.7 \%$ with a relative risk of developing CVC-related thrombosis of 2.6-7.7. The prothrombin G20210A mutation suggests to be associated (RR 2.3) with CVC-related thrombosis in cancer patients. Congenital deficiencies in protein $\mathrm{C}$, protein $\mathrm{S}$ or antithrombin are rare and their potential role on the occurrence of CVC-related thrombosis in cancer patients is unknown. Patients with a malignant disease exhibit higher plasma levels of homocysteine compared to the general population. The limited evidence available suggests a higher risk of CVC-related thrombosis in patients with a CVC and elevated plasma levels of homocysteine. A family history of venous thromboembolism gives a relative risk of developing CVC-related thrombosis of 1.3. Routine screening or screening of specific groups of cancer patients with a CVC for congenital thrombophilia cannot be recommended on the basis of the studies discussed. 
In chapter 6 we aimed to find, in a prospective setting, associations between biomarkers of the hemostatic system and the occurrence of CVC-related thrombosis in hematological patients undergoing intensive chemotherapy. We have chosen for easy to determine biomarkers from the risk-model of Khorana (pre chemotherapy platelet count, leukocyte count, hemoglobin level) [11] in combination with factor VIII (FVIII), plasminogen activator inhibitor-1 (PAI-1), activated protein C (APC) resistance and free protein S antigen. From 168 patients, 4 tubes of citrated plasma were collected. Nine percent developed a CVC-related thrombosis. We identified elevated white blood cell count, factor VIII, and PAI-1 to be risk factors for developing symptomatic CVC-related thrombosis in patients with hematological malignancies.

Seven from the 15 patients (47\%) with a symptomatic CVC-related thrombosis had a leukocyte count above the $75^{\text {th }}$ percentile $\left(12.6 * 10^{9} /\right.$ I) compared to $35 / 153(23 \%)$ patients without a symptomatic CVC-related thrombosis ( $p=0.042$ ).

Mean factor VIII was $170 \%$. Patients that developed a CVC-related thrombosis ( $n=$ 15: mean 203\%; 8-415\%) had significantly higher factor VIII levels at baseline than those who stayed free of events during follow up ( $n=153$; mean 166\%: $110-293 \%$; $P=0.023$ ). Factor VIII did not differ according to the type of hematological malignancy $(p=0.214)$.

PAI-1 activity could be determined for 167 patients. The median PAI-1 activity in the symptomatic CVC-related group was $12.2 \mathrm{IU} / \mathrm{ml}$ (IQR 3.5-22.7 IU/ml) and the median PAI-1 activity in the group without a CVC-related thrombosis was $4.9 \mathrm{IU} / \mathrm{ml}$ (IQR 1.7$10.2 \mathrm{lU} / \mathrm{ml})(p=0.062)$. When setting the cut off level for elevated PAl-1 at the $75^{\text {th }}$ percentile of the total study population we found statistically significant more patients with high PAI-1 in the group with CVC-related thrombosis (8/15 patients, 53\%) compared to the group without CVC-related thrombosis $(34 / 152,22 \% ; p=0.008)$. 


\section{REFERENCES}

1. McGee DC, Gould MK. Preventing complications of central venous catheterization. N Engl J Med 2003; 348: 1123-1133.

2. Jarvis WR. Selected aspects of the socioeconomic impact of nosocomial infections: morbidity, mortality, cost, and prevention. Infect Control Hosp Epidemiol 1996; 17: 552-557.

3. Zwicker J, Connolly G, Carrier M et al. Catheter-associated deep vein thrombosis of the upper extremity in cancer patients: guidance from the SSC of the ISTH. J Thromb Haemost 2014; 12 (5): 796-800.

4. Schiffer CA, Mangu PB, Wade JC et al. Central venous catheter care for the patient with cancer: American Society of Clinical Oncology clinical practice guideline. J Clin Oncol 2013; 31 (10): 1357-1370.

5. Abdelkefi A, Torjman L, Ladeb $S$ et al. Randomized trial of prevention of catheter-related bloodstream infection by continuous infusion of low-dose unfractionated heparin in patients with hematologic and oncologic disease. J Clin Oncol 2005; 23: 7864-7870.

6. Lordick $F$, Hentrich $M$, Decker $T$ et al. Ultrasound screening for internal jugular vein thrombosis aids the detection of central venous catheter-related infections in patients with haemato-oncological diseases: a prospective observational study. Br J Haematol 2003; 120: 1073-1078.

7. van Rooden CJ, Schippers EF, Barge RM et al. Infectious complications of central venous catheters increase the risk of catheter-related thrombosis in hematology patients: a prospective study. J Clin Oncol 2005; 23: 2655-2660.

8. Mehall JR, Saltzman DA, Jackson RJ, Smith SD. Fibrin sheath enhances central venous catheter infection. Crit Care Med 2002; 30: 908-912.

9. Weijmer MC, Debets-Ossenkopp YJ, Van De Vondervoort FJ, ter Wee PM. Superior antimicrobial activity of trisodium citrate over heparin for catheter locking. Nephrol Dial Transplant 2002; 17: 2189-2195.

10. Weijmer MC, van den Dorpel MA, Van de Ven PJ et al. Randomized, clinical trial comparison of trisodium citrate $30 \%$ and heparin as catheter-locking solution in hemodialysis patients. J Am Soc Nephrol 2005; 16: 2769-2777.

11. Khorana AA, Kuderer NM, Culakova E et al. Development and validation of a predictive model for chemotherapy-associated thrombosis. Blood 2008; 111: 4902-4907. 


\section{NEDERLANDSTALIGE SAMENVATTING}

Dit proefschrift gaat over late complicaties van centraal veneuze catheters (CVC's) in patiënten met een hematologische ziekte die intensieve chemotherapie krijgen. CVC's worden veelvuldig gebruikt in deze categorie patiënten om bloed af te nemen voor diagnostiek en om chemotherapie, stamcelinfusies, bloedproducten, medicatie en voeding toe te kunnen dienen.

Complicaties van deze CVC's bestaan uit mechanische complicaties tijdens of direct na het inbrengen van de CVC en late complicaties zoals infecties en tromboses [1]. Centraal veneuze catheter-gerelateerde infecties en tromboses leiden tot morbiditeit, verlenging van de opnameduur en kosten [2]. Vele studies hebben de incidentie en risicofactoren voor CVC-gerelateerde infecties en tromboses onderzocht in patiënten met een solide tumor, maar slechts weinig data zijn bekend over hematologische patiënten met een CVC die intensieve chemotherapie ondergaan.

Een algemene inleiding en overzicht van het proefschrift wordt in hoofdstuk 1 gegeven.

In hoofdstuk 2 wordt een systematisch overzicht gegeven van de gepubliceerde studies betreffende CVC-gerelateerde complicaties in hematologische patiënten die intensieve chemotherapie ondergaan. De incidentie, pathogenese, risicofactoren, diagnose, behandeling en preventieve maatregelen worden beschreven. De incidentie van een symptomatische CVC-gerelateerde trombose varieert tussen de 1.2-18.0\%.

Een longembolie treedt op in 15-25\% van de patiënten met een CVC-gerelateerde trombose, een post-trombotische arm in $14.8 \%$ van de patiënten met een CVCgerelateerde trombose en er is een verhoogd risico op CVC-gerelateerde infecties. De huidige richtlijnen adviseren niet om profylactische antistolling te geven met als doel het risico op CVC-gerelateerde tromboses te verkleinen.

De incidentie van CVC-gerelateerde bacteriëmien varieert in studies tussen de 0.040.6\%. CVC-gerelateerde infecties in hematologische patiënten worden meestal veroorzaakt door coagulase-negatieve stafylokokken (62.5\%), gram negatieve bacteriën zoals Enterobacter species, Escherichia coli en Pseudomonas species (29\%) of Candida species (4.2\%). De diagnose CVC-gerelateerde bacteriëmie (CVC-BSI) stellen is soms lastig. Er is behoefte aan een specifieke aanpak ten aanzien van de diagnostiek en de behandeling van een CVC-gerelateerde bacteriëmie en/of trombose met aandacht voor het behouden van de CVC.

CVC-gerelateerde tromboses en infecties hebben een relatie met elkaar [6, 7]. Interventies die er op gericht zijn fibrine depositie en trombus vorming in de CVC te voorkomen kunnen daarmee zowel de incidentie van CVC-gerelateerde tromboses als infecties verminderen.

De resultaten van een vragenlijst onder 23 Nederlandse en Belgische hematologische centra die intensieve chemotherapie volgens de HOVON geven worden 
besproken in hoofdstuk 3. De vragenlijst bevatte 30 vragen over de lokale gebruiken en protocollen betreffende type CVC, insertie en verzorging van de CVC, alsmede diagnose, behandeling en preventie van CVC-gerelateerde tromboses en infecties. De vragenlijst werd gestuurd naar een ervaren hematoloog in elke centrum. De gebruiken en protocollen variëren sterk wat waarschijnlijk een algemeen gebrek aan standaardisatie in beleid aangeeft in Europa.

Fibrinevorming rond de buitenkant van de CVC en in het lumen is een belangrijke risicofactor voor zowel tromboses als infecties [8]. Geconcentreerd citraat (TSC) als lock voorkomt CVC-gerelateerde tromboses door middel van chelatie van geïoniseerd calcium. TSC heeft brede antimicrobiële en anti gist effecten [9]. TSC verlaagt de incidentie van CVC-gerelateerde bacteriëmien (BSI) in hemodialyse patiënten van 4.1 CVC-gerelateerde BSI per 1000 catheter dagen naar 1.1 per 1000 catheter dagen [10]. Derhalve hebben wij een gerandomiseerde trial opgezet in hematologische patiënten die intensieve chemotherapie ondergaan om de effecten van locken met TSC op CVCgerelateerde tromboses en infecties te onderzoeken ten op zichte van locken met heparine. De resultaten zijn beschreven in hoofdstuk 4. Vier en dertig CVC-BSI werden gevonden in de 108 patiënten die heparine als lock kregen en 35 CVC-BSI werden gevonden in de 99 patiënten die hun CVC met citraat gelocked kregen $(P=0.654)$. Zeven maal zoveel CVC-BSI met gram negatieve staven in de CVCs die gelocked werden met heparine $(p=0.041)$ werden gezien. De cumulatieve incidentie van symptomatische CVC-gerelateerde tromboses was $10 \%$ in de heparine groep en $5 \%$ in de TSC groep (hazard ratio 0.525; 95\% confidentie interval 0.182-1.512).

Een incidentie van 60\% CVC-BSI werd gevonden in patiënten die intensieve chemotherapie ondergingen vergeleken met een incidentie van 7\% CVC-BSI in patiënten die een autologe stamceltransplantatie ondergingen.

In hoofdstuk 5 wordt een overzicht gegeven van de belangrijkste studies die onderzochten of trombofilie het risico op een CVC-gerelateerde trombose in patiënten met een maligniteit vergroot. Een uitgebreid overzicht van de studies die tussen 1990 en 2010 gepubliceerd zijn, werd gemaakt met behulp van de MEDLINE database. De prevalentie van factor $V$ Leiden in patiënten met een maligniteit varieert van 4.9-9.7\% met een relatief risico op het ontwikkelen van een CVC-gerelateerde trombose van 2.67.7. De protrombine mutatie lijkt een relatief risico van 2.3 te geven op het ontwikkelen van een CVC-gerelateerde trombose in patiënten met een maligniteit. Congenitale deficiënties van proteine $C$, proteine $S$ of antitrombine zijn zeldzaam en hun rol in het ontwikkelen van CVC-gerelateerde tromboses in patiënten met een maligniteit is niet duidelijk.

Patiënten met een maligniteit hebben een hoger homocysteine gehalte vergeleken met gezonde personen. Het beperkt aantal gepubliceerde studies suggereert een hoger risico op CVC-gerelateerde tromboses in patiënten met een verhoogd homocysteine gehalte en een CVC. Een positieve familie anamnese voor veneuze tromboses geeft een relatief risico van 1.3 op het ontwikkelen van een CVC-gerelateerde trombose. 
Routinematig testen op trombofilie in patiënten met een maligniteit die een CVC krijgen kan niet worden aangeraden op dit moment.

In hoofdstuk 6 kijken we in een prospectieve studie naar associaties tussen biomarkers van het hemostatische systeem en het optreden van CVC-gerelateerde tromboses in hematologische patiënten die intensieve chemotherapie ondergaan. We hebben gekozen voor eenvoudig te bepalen biomarkers van het risico model van Khorana (pre chemotherapie trombocyten aantal, leukocyten getal, hemoglobine) [11] in combinatie met factor VIII (FVIII), plasminogeen activator inhibitor-1 (PAI-1), geactiveerd proteine C (APC) resistentie en vrij proteine $S$ antigeen. Van 168 patiënten is bloed verzameld. Negen procent van de patiënten ontwikkelde een CVC-gerelateerde trombose. We vonden leukocytose, verhoogd factor VIII, and PAI-1 als risico factor voor het ontwikkelen van een CVC-gerelateerde trombose in patiënten met een hematologische ziekte.

Zeven van de 15 patiënten (47\%) met een symptomatische trombose hadden een leukocyten getal boven de $75^{\mathrm{e}}$ percentiel $\left(12.6 * 10^{9} / \mathrm{l}\right)$ vergeleken met 35/153 (23\%) van de patiënten zonder een CVC-gerelateerde trombose $(p=0.042)$.

Mean factor VIII gehalte was $170 \%$. Patiënten die een CVC-gerelateerde trombose ontwikkelden ( $n=15$; mean 203\%; 8-415\%) hadden significant hogere factor VIII waardes dan de patiënten die geen CVC-gerelateerde trombose ontwikkelden ( $n=153$; mean 166\%; 110-293\%; $P=0.023)$. Factor VIII waardes verschilden niet per ziektebeeld $(p=0.214)$.

PAI-1 activiteit werd voor 167 patiënten bepaald. De mediane PAI-1 activiteit in de groep patiënten met een symptomatische CVC-gerelateerde trombose was $12.2 \mathrm{IU} / \mathrm{ml}$ (IQR 3.5-22.7 IU/ml). De mediane PAl-1 activiteit in de groep zonder trombose was 4.9 $\mathrm{IU} / \mathrm{ml}(\mathrm{IQR}$ 1.7-10.2 IU/ml) ( $\mathrm{p}=0.062)$. Bij het zetten van de cut-off level voor verhoogde PAI-1 activiteit op de $75^{\mathrm{e}}$ percentiel van de totale studie populatie vonden we statistisch significant meer patiënten met een hoge PAI-1 in de groep met een CVCgerelateerde trombose (8/15 patiënten, 53\%) vergeleken met de groep zonder CVC gerelateerde trombose $(34 / 152,22 \% ; p=0.008)$. 


\section{REFERENTIES}

1. McGee DC, Gould MK. Preventing complications of central venous catheterization. N Engl J Med 2003; 348: 1123-1133.

2. Jarvis WR. Selected aspects of the socioeconomic impact of nosocomial infections: morbidity, mortality, cost, and prevention. Infect Control Hosp Epidemiol 1996; 17: 552-557.

3. Zwicker J, Connolly G, Carrier M et al. Catheter-associated deep vein thrombosis of the upper extremity in cancer patients: guidance from the SSC of the ISTH. J Thromb Haemost 2014; 12 (5): 796-800.

4. Schiffer CA, Mangu PB, Wade JC et al. Central venous catheter care for the patient with cancer: American Society of Clinical Oncology clinical practice guideline. J Clin Oncol 2013; 31: 1357-1370.

5. Abdelkefi A, Torjman L, Ladeb S et al. Randomized trial of prevention of catheter-related bloodstream infection by continuous infusion of low-dose unfractionated heparin in patients with hematologic and oncologic disease. J Clin Oncol 2005; 23: 7864-7870.

6. Lordick F, Hentrich M, Decker T et al. Ultrasound screening for internal jugular vein thrombosis aids the detection of central venous catheter-related infections in patients with haemato-oncological diseases: a prospective observational study. Br J Haematol 2003; 120: 1073-1078.

7. van Rooden CJ, Schippers EF, Barge RM et al. Infectious complications of central venous catheters increase the risk of catheter-related thrombosis in hematology patients: a prospective study. J Clin Oncol 2005; 23: 2655-2660.

8. Mehall JR, Saltzman DA, Jackson RJ, Smith SD. Fibrin sheath enhances central venous catheter infection. Crit Care Med 2002; 30: 908-912.

9. Weijmer MC, Debets-Ossenkopp YJ, Van De Vondervoort FJ, ter Wee PM. Superior antimicrobial activity of trisodium citrate over heparin for catheter locking. Nephrol Dial Transplant 2002; 17: 2189-2195.

10. Weijmer MC, van den Dorpel MA, Van de Ven PJ et al. Randomized, clinical trial comparison of trisodium citrate 30\% and heparin as catheter-locking solution in hemodialysis patients. J Am Soc Nephrol 2005; 16: 2769-2777.

11. Khorana AA, Kuderer NM, Culakova E et al. Development and validation of a predictive model for chemotherapy-associated thrombosis. Blood 2008; 111: 4902-4907. 


\section{DANKWOORD}

Het is af...... Na 10 (!) jaren in de schaarse vrije avonduren, weekeinden, vakanties en zwangerschapsverloven achter mijn laptop op de eettafel te hebben gezeten, ben ik eindelijk aangekomen bij het wellicht meest danwel door sommigen (mijn man) enige gelezen gedeelte van mijn proefschrift.

Promoveren kan je zeker niet alleen. Zonder steun van de mensen om mij heen hadden mijn inspanningen nooit kunnen resulteren in dit boekje. Daarom wil ik iedereen die heeft bijgedragen aan dit proefschrift hartelijk bedanken en sommige mensen in het bijzonder noemen.

Professor dr. H. C. Schouten, beste Harry, als promotor waakte je over de inhoud en voortgang van mijn proefschrift. Hoorde je te lang niets, dan kwam er een 'motiverend' belletje, smsje of mailtje. Jarenlang op rij kreeg ik met nieuwjaar een berichtje dat dit jaar vast het jaar zou worden waarin ik zou promoveren. Voor mijn $35^{\mathrm{e}}$ verjaardag kreeg ik een nieuwe licentie van SPSS cadeau van je... Dank voor jouw vertrouwen, hulp en inspiratie; niet alleen voor dit proefschrift, maar ook voor de fijne opleidingsjaren in Maastricht waar ik nog steeds met veel plezier op terugkijk.

Dr. K. Hamulyak, beste Karly. Dank voor al jouw overwegingen, kritische noten en de vele, vele artikelen die jij mij steeds 'ter info' stuurde.

Beste Asiong, door jou is mijn grote interesse en liefde voor de hematologie ontstaan en mede door jou is dit onderzoek opgezet. Dank voor beiden!

Prof. Dr. A. Verbon, Beste Annelies; wat werkt jouw enthousiasme aanstekelijk! Dank voor het meedenken op infectieus gebied, eerst vanuit Maastricht en later vanuit Rotterdam.

Adri Voogd en Attie Tuinenburg, de kritische blik waarmee jullie de statistiek in mijn artikelen beoordeelden hebben enorm bijgedragen aan mijn kennis en begrip van statistiek.

Prof. Dr. G. M. J. Bos, Prof. Dr. C. D. A. Stehouwer, Dr. M. R. Nijziel en Dr. P. J. Voogt, dank voor jullie bereidheid om mijn proefschrift op wetenschappelijke waarde te beoordelen en zitting te nemen in de promotiecommissie. Beste Gerard en Paul, tevens dank voor alles wat ik van jullie heb geleerd tijdens mijn opleiding en met name voor de prettige en constructieve manier waarop jullie dit hebben gedaan.

Claudia, Mariëlle en Ellen, dank voor het informeren, includeren en randomiseren van alle patiënten.

Verpleging van afdeling A5 in Maastricht en de verpleging Interne Geneeskunde/ hemato-oncologie in Heerlen, dank voor het locken van alle lumina en het invullen van al die formulieren.

Dames en heren op het stollingslab, dank voor het uitvoeren van alle testen.

Rene van Oerle en Arina ten Cate, wat fijn dat jullie kritisch meedachten. 
Beste maten van de vakgroep Interne geneeskunde/MDL van het Amphia ziekenhuis en in het bijzonder beste Joost, Marjolein, Bert-Jan, Olaf, Joan en Hans: Wat hebben we een mooie praktijk met elkaar samen! 'Anne-Fleur' heeft na een hele lange draagtijd dan toch eindelijk het levenslicht gezien! Dank voor jullie niet aflatende interesse en aanmoedigingen in de vorderingen van de uitdrijving van mijn proefschrift. Alexander.....

Lieve Kirsten, wat hebben wij samen een leuke opleidingstijd op de afdeling hematologie in Maastricht gehad. Ik hoop dat we nog vele (zonnige) nascholingen samen plannen want nascholing met jou erbij is zoveel gezelliger! Wat fijn dat je naast me staat als paranimf. Lieve Vanessa, dank dat jij als dierbare vriendin aan de andere kant van mij als paranimf staat. Je bent altijd in Club Med Wouw welkom!

Lieve familie en vrienden, dank voor jullie interesse, jullie steun, jullie liefde en jullie gezelligheid!

Tot slot (maar eigenlijk zou ik jullie als eerste moeten noemen), lieve Barthold, Isabelle, Josephine en Casper; wat ben ik trots op onze fijne gezin! Jullie houden mijn focus op wat er uiteindelijk echt toe doet. 


\section{CURRICULUM VITAE}

Rinske Boersma werd op 4 september 1978 geboren in Velp. In 1996 behaalde zij haar VWO diploma aan het Christelijk Lyceum in Arnhem. In datzelfde jaar startte zij met de studie geneeskunde aan de Universiteit van Maastricht, waar zij in 1997 de propedeuse behaalde. In 2002 behaalde zij haar artsexamen met het predicaat Cum Laude. Na het behalen van het artsexamen heeft zij 6 maanden als arts-assistent niet in opleiding op de afdeling gynaecologie en verloskunde in het Laurentius ziekenhuis in Roermond gewerkt en hierna 6 maanden als arts-assistent niet in opleiding op de afdeling interne geneeskunde in het Atrium Medisch Centrum in Heerlen (thans Zuyderland Medisch Centrum). In 2003 startte zij met de opleiding tot internist in het Atrium Medisch Centrum in Heerlen (opleider Dr. P. Voogt) waarna ze deze vanaf 2007 voortzette in het Academisch Ziekenhuis Maastricht (thans: Maastricht Universitair Medisch Centrum) (opleider: Prof. dr. C. D. A. Stehouwer). In 2007 ving ook de opleiding tot hematoloog aan (opleider: Prof. dr. H. C. Schouten). In juni 2010 volgde haar registratie als internisthematoloog. Sinds juni 2010 werkt zij met heel veel plezier als internist-hematoloog in het Amphia ziekenhuis in Breda. In 2005 begon zij aan de promotie over centraal veneuze katheter-gerelateerde complicaties in hematologische patiënten.

Rinske is getrouwd met Barthold Kuiken en samen hebben zij drie kinderen, Isabelle (2007), Josephine (2009) en Casper (2012). 



\section{LIST OF PUBLICATIONS}

Boersma RS, Hamulyak K, van Oerle R, Tuinenburg A, Cate-hoek AJ, H.C. Schouten. Biomarkers for prediction of central venous catheter-related-thrombosis in patients with hematological malignancies. Clin Appl Thromb Hemost. 2015 Apr 17 (epub ahead of print)

Van Gammeren AJ, Alcala LS, Smolders M, Boersma RS. Numerous Russell bodies and Dutcher bodies in multiple myeloma. Br J Haematol. 2015 Sep;170(6):743

Boersma RS, Jie KS, Voogd AC, Hamulyak K, Verbon A, Schouten HC. Concentrated citrate locking in order to reduce the long-term complications of central venous catheters: a randomized controlled trial in patients with hematological malignancies. Support Care Cancer. 2015 Jan;23(1): 37-45

Stevens KN, Croes C, Boersma RS, Stoberingh EE, van der Marel C, van der Veen FH, Knetsch ML, Koole LH. Hydrophylic surface coatings with embedded biocidal silver nanoparticles and sodium heparin for central venous catheters. Biomaterials. 2011 Feb; 32(5):1264-9

Boersma RS, Hamulyak K, Cate HT, Schouten HC. Congenital thrombophilia and central venous catheter-related thrombosis in patients with cancer. Clin Appl Thromb Hemost. 2010 Dec; 16(6):643-9

Boersma RS, Schouten HC. Clinical practices concerning central venous catheters in haematological patients. Eur J Oncol Nurs. 2010 Jul;14(3):200-4

Boersma RS, Jie KS, Verbon A, van Pampus EC, Schouten HC. Thrombotic and infectious complications of central venous catheters in patients with hematological malignancies. Ann Oncol. 2008. Mar;19(3);433-42

Boersma RS, Buijs J. Simultaneous bilateral spontaneous pneumothorax as a presenting symptom of osteosarcoma. Eur J Inter Med. 2007 Jul;18(4);342-3 



\section{VALORIZATION}

Central venous catheters (CVCs) are extensively used in patients with hematological malignancies undergoing intensive chemotherapy to provide cancer treatment and supportive care therapies. These CVCs improve patients' quality of life by reducing the need for venipunctures and allowing patients to receive chemotherapy, stem cell infusions, blood products, medication and parenteral nutrition. CVCs have become a 'life line' for hematological patients. The great benefits can be offset by complications. Reported complications consist of mechanical complications during or directly after the insertion (arterial puncture, hematoma and pneumothorax) and long-term complications like CVC-related infections and thrombosis. The incidence of CVC-related thrombosis varies between $1.2-18 \%$ and the incidence of CVC-related bloodstream infections varies between 0.0-40.6\%. CVC-related infections and thrombosis impair CVC functioning and result in patient morbidity, may interrupt or delay administration of cancer therapy and represent a costly burden to the healthcare system. Long term complications pose hematologists with difficult questions on what anticoagulant treatment to choose in often thrombocytopenic patients and whether the CVC ('lifeline') must be removed. The total annual costs of CVC-related infections in patients with cancer in the United States is estimated to exceed 18 billion dollars.

This thesis is about long term complications of central venous catheters in hematological patients undergoing intensive chemotherapy. Based on above information we can conclude that CVC-related infections and thrombosis in hematological patients undergoing intensive chemotherapy are clinically relevant problems causing morbidity, mortality and costs for the health care system. They should not be accepted as complications of modern care but must be one of the priority targets of a multidisciplinary approach emphasizing quality-of-care improvement. This thesis may help improving quality of care and thereby reducing morbidity, mortality and costs.

Many studies have addressed the incidence and associated risk factors of CVCrelated thrombosis and infections in patients with solid tumors, but only limited data are available on hematological patients. This thesis focuses exclusively on hematological patients with a CVC undergoing intensive chemotherapy. Since these patients are often thrombocytopenic and leucopenic, experience mucositis and their CVC is considered a life line they deserve a specific approach. This thesis provides insight into this specific group of patients and why these patients deserve a specific approach. The ultimate target should be a multidisciplinary approach and strict adherence to an evidence based guideline. This thesis with its extensive review and studies focused specific on hematological patients provides a basis for the development of such a guideline.

Prevention of the long term complications is of utmost importance. The paradigm for dealing with these long-term complications needs to shift from treatment to 
prevention. Guidelines recommend against the routine administration of pharmacologic prophylaxis to prevent CVC-related thrombosis. Since fibrin sheath formation around the external portion of the CVC and within the catheter lumen has been implicated as a major contributing factor in both occlusive and infectious events we started our randomized controlled study to determine the efficacy of concentrated citrate as a locking solution compared to heparin. No differences in CVC-related BSI were found. We did find seven times more CVC-BSI with gram negative organisms in the heparin group and a not statistically significant doubling of the incidence of CVC-related thrombosis in the heparin group. These are findings that warrant further investigation.

In this thesis elevated leukocyte count, factor VIII and plasminogen activator inhibitor-1 were found to be potential interesting biomarkers. Further investigation determining baseline clinical and laboratory parameters to help identify hematological patients at highest risk for CVC-related thrombosis and infections is needed. This will help to tailor the management of central venous catheters in hematological patients undergoing intensive chemotherapy. 
